\title{
Kaon Decays in the Standard Model
}

\author{
Vincenzo Cirigliand* \\ Theoretical Division, Los Alamos National Laboratory, Los Alamos, NM 87545, USA \\ Gerhard Ecker $\dagger^{\dagger}$ and Helmut Neufeld \\ University of Vienna, Faculty of Physics, Boltzmanngasse 5, A-1090 Wien, Austria \\ Antonio Pich $\$$ and Jorge Portolés \\ Departament de Física Teòrica, IFIC, Universitat de València - CSIC, \\ Apartat de Correus 22085, E-46071 València, Spain
}

\begin{abstract}
A comprehensive overview of kaon decays is presented. The Standard Model predictions are discussed in detail, covering both the underlying short-distance electroweak dynamics and the important interplay of QCD at long distances. Chiral perturbation theory provides a universal framework for treating leptonic, semileptonic and nonleptonic decays including rare and radiative modes. All allowed decay modes with branching ratios of at least $10^{-11}$ are analyzed. Some decays with even smaller rates are also included. Decays that are strictly forbidden in the Standard Model are not considered in this review. The present experimental status and the prospects for future improvements are reviewed.
\end{abstract}

\section{Contents}

\section{Introduction}

II. Theoretical framework

A. Short-distance description

B. Chiral perturbation theory

III. Estimates of low-energy constants

A. The large- $\mathbf{N}_{\mathbf{C}}$ limit of QCD

B. Lattice determinations

IV. Leptonic and semileptonic decays

A. $K_{\ell 2}\left(\right.$ and $\left.\pi_{\ell 2}\right)$ decays

1. Electromagnetic corrections

2. Extraction of $V_{u s} / V_{u d}$

3. The ratio $R_{e / \mu}^{(K, \pi)}$

B. $K_{\ell 2 \gamma}$

C. $K_{\ell 3}$

1. Electromagnetic effects in $K_{\ell 3(\gamma)}$ decays

2. Quark mass ratios and $K_{\ell 3}$ decays

3. Form factors and phase space integrals

4. The $K_{\ell 3}$ scalar form factor

5. SU(3) breaking effects in $f_{+}^{K^{0} \pi^{-}}(0)$

6. Determination of $V_{u s}$ and CKM unitarity tests

7. $\mathrm{T}$ violation in $K_{\mu 3}$ decays

D. $K_{\ell 3 \gamma}$

E. $K_{\ell 4}$

F. $K_{e 5}$

V. Nonleptonic decays

A. $K \rightarrow \pi \pi$

1. $\pi \pi$ phase shifts from $K \rightarrow \pi \pi$ decays

2. $\epsilon^{\prime} / \epsilon$

3. $\mathrm{CP}$ violation in $K^{0}-\overline{K^{0}}$ mixing
B. $K \rightarrow 3 \pi$

1. CP violation in $K \rightarrow 3 \pi$ decays

2. $\pi \pi$ scattering lengths from $K \rightarrow 3 \pi$ decays near threshold

VI. Rare and radiative decays

A. $K \rightarrow \pi \nu \bar{\nu}, \pi \pi \nu \bar{\nu}$

B. $K \rightarrow \gamma^{(*)} \gamma^{(*)}$

1. $K_{S} \rightarrow \gamma \gamma$

2. $K_{L} \rightarrow \gamma \gamma$

3. $K_{S} \rightarrow \gamma \ell^{+} \ell^{-}$

4. $K_{L} \rightarrow \gamma \ell^{+} \ell^{-}$

5. $K_{L} \rightarrow \ell_{1}^{+} \ell_{1}^{-} \ell_{2}^{+} \ell_{2}^{-}$

C. $K \rightarrow \ell^{+} \ell^{-}$

1. $K_{S} \rightarrow \ell^{+} \ell^{-}$

2. $K_{L} \rightarrow \ell^{+} \ell^{-}$

D. $K \rightarrow \pi \gamma \gamma^{(*)}$

1. $K^{+} \rightarrow \pi^{+} \gamma \gamma$

2. $K_{S} \rightarrow \pi^{0} \gamma \gamma$

3. $K_{L} \rightarrow \pi^{0} \gamma \gamma$

4. $K \rightarrow \pi \gamma \ell^{+} \ell^{-}$

E. $K \rightarrow \pi \ell^{+} \ell^{-}$

1. $K_{S}, K^{ \pm} \rightarrow \pi \ell^{+} \ell^{-}$

2. $K_{L} \rightarrow \pi^{0} \ell^{+} \ell^{-}$

F. $K \rightarrow \pi \pi \gamma^{(*)}$

1. $K^{+} \rightarrow \pi^{+} \pi^{0} \gamma$

2. $K_{L} \rightarrow \pi^{+} \pi^{-} \gamma$

3. $K_{S} \rightarrow \pi^{+} \pi^{-} \gamma$

4. $K \rightarrow \pi \pi \ell^{+} \ell^{-}$

G. Other decays

1. $K^{0} \rightarrow \gamma \gamma \gamma$

2. $K_{L} \rightarrow \gamma \gamma \ell^{+} \ell^{-}$

3. $K_{L} \rightarrow \gamma \nu \bar{\nu}$

4. $K_{S} \rightarrow \ell_{1}^{+} \ell_{1}^{-} \ell_{2}^{+} \ell_{2}^{-}$

5. $K_{L}, K_{S} \rightarrow \pi^{0} \pi^{0} \gamma$

6. $K_{L}, K_{S} \rightarrow \pi^{0} \pi^{0} \gamma \gamma$

7. $K \rightarrow 3 \pi \gamma$

VII. Conclusions and outlook

Acknowledgments

${ }^{\dagger}$ Electronic address: gerhard.ecker@univie.ac.at

‡Electronic address: helmut.neufeld@univie.ac.at

$\S$ Electronic address: Antonio.Pich@ific.uv.es

๑Electronic address: Jorge.Portoles@ific.uv.es
A. One-loop functions

References 


\section{INTRODUCTION}

Kaon decays have played a key role in the shaping of the Standard Model (SM) (Glashow, 1961; Salam, 1968; Weinberg, 1967), from the discovery of kaons (Rochester and Butler 1947) until today. Prominent examples are the introduction of strangeness (Gell-Mann, 1953, Pais, 1952), parity violation ( $K \rightarrow 2 \pi, 3 \pi$ puzzle) (Dalitz, 1954 Lee and Yang, 1956), quark mixing (Cabibbo, 1963, Kobayashi and Maskawa, 1973), the discovery of CP violation (Christenson et al. , 1964), suppression of flavorchanging neutral currents (FCNC) and the GIM mechanism (Glashow et al. 1970). Moreover, kaon decays continue to have an important impact on flavor dynamics in constraining physics beyond the SM.

The aim of this review is a comprehensive survey of kaon decays allowed in the SM with branching ratios of at least $10^{-11}$. Some decays are included with even smaller decay rates. We do not cover decays that are strictly forbidden in the SM such as lepton-number or leptonflavor violating decays.

Kaon decays involve an intricate interplay between weak, electromagnetic and strong interactions. A major theoretical challenge has to do with the intrinsically nonperturbative nature of the strong interactions in kaon physics. The last 25 years have seen the development of a systematic approach to low-energy hadron physics in the framework of chiral perturbation theory (we refer to Sec. II.B for references). The approach provides a systematic expansion of decay amplitudes in terms of momenta and meson masses. The momenta of particles in the final states are sufficiently small so that the kaon mass sets the scale for the quality of the expansion. The relevant dimensionless ratio is $M_{K}^{2} /\left(4 \pi F_{\pi}\right)^{2} \simeq 0.18$.

Looking in more detail into the predictions of chiral perturbation theory (CHPT) for kaon decays, one observes a rather wide range, from cases where great precision can be achieved [especially in (semi)leptonic decays] to processes where hadronic uncertainties remain the major obstacle for comparison with experiment (mainly in nonleptonic decays). However, an important feature of CHPT in general is that it parametrizes the intrinsic hadronic uncertainties by a number of parameters, the so-called low-energy constants (LECs). The quality of theoretical predictions hinges to a large extent on the available information on those LECs. Over the years, a lot of progress has been achieved in the theoretical understanding and the phenomenological knowledge of LECs. In recent years, lattice simulations have made important contributions to this field. For the time being, lattice QCD gives access mainly to strong LECs (see Sec. III). This is one of the reasons why much better precision can be achieved in semileptonic than in nonleptonic decays.

Kaon decays form a fascinating chapter of particle physics in themselves but they also give access to fundamental parameters of the SM such as the CabibboKobayashi-Maskawa (CKM) matrix elements $V_{u s}$ and $V_{t d}$. Although we stay strictly within the SM for this review, kaon decays have of course the potential to catch glimpses of New Physics, especially in those cases where the SM makes precise predictions. The constraints on New Physics are most effective in combination with other information from the low-energy/high-intensity frontier that are out of scope for this review. However, the impact of such constraints depends crucially on the quality of SM predictions. A state-of-the-art survey of kaon decays in the SM is therefore expected to be very useful also for physics beyond the SM.

We review the status of $K$ decays at a time when both theory and experiment progress at a somewhat slower pace than ten years ago. Nevertheless, there is still considerable activity in the field. The experimental program for kaon decays concentrates on specific channels of particular interest for physics beyond the SM but results for other channels can be expected as by-products. The main players in the near future on the experimental side will be NA62 at CERN (Collazuol, 2009), K0 TO (Nanjo, 2009) and TREK (Kohl, 2010; Paton et al., 2006) at J-PARC, KLOE-2 at DA $\Phi$ NE (Amelino-Camelia et al. , 2010), KLOD (Bolotov et al., 2009) and OKA (Kurshetsov, 2009) at IHEP Protvino, and the proposed Project-X at Fermilab (Bryman and Tschirhart, 2010).

Kaon decays have been treated in several reviews and lecture notes during the past 20 years (Artuso et al. 2008; Barker and Kettell, 2000, Battiston et al., 1992; Bryman, 1989 Buchalla, 2001; Buchholz and Renk, 1997; Buras, 1996, Buras et al., 2008; D'Ambrosio et al., 1994; Littenberg and Valencia, 1993; Ritchie and Wojcicki, 1993 , Winstein and Wolfenstein, 1993).

The review is organized along the following lines. We start with a brief summary of the theoretical framework, including both the short-distance aspects and the lowenergy realization in terms of CHPT. For the latter, we also recapitulate the present knowledge of the coupling constants in the chiral Lagrangians (LECs). As already emphasized and in contrast to most previous reviews of the field, we then discuss essentially all kaon decays allowed in the SM that have either already been measured or that may become accessible experimentally in the not too distant future. We divide the actual review of different channels into three parts: leptonic and semileptonic decays, dominant nonleptonic decays $(K \rightarrow 2 \pi, 3 \pi)$ and, finally, rare and radiative decays.

In Sec. II, we recall the effective Lagrangians in the SM, for both semileptonic and nonleptonic decays, after integrating out the $W$ and $Z$ bosons and the heavy quarks $(t, b, c)$. For nonleptonic transitions, the leading QCD corrections are summed up with the help of the operator product expansion (OPE) and the renormalization group. The second part of this section contains a brief introduction to CHPT, concentrating on the various chiral Lagrangians relevant for $K$ decays. Estimates of LECs are presented in Sec. III. In addition to phenomenological determinations, the large- $N_{C}$ limit of QCD provides a useful framework for theoretical estimates. Strong LECs are dominated by the exchange of meson resonances. The re- 
sulting numerical estimates are in general in good agreement with upcoming lattice determinations. Through the hadronization of short-distance operators appearing in the effective Lagrangian for nonleptonic transitions at the quark level, electroweak LECs can be expressed in terms of strong and electromagnetic couplings.

In Sec. IV] we review the current status of leptonic and semileptonic modes (including radiative channels). $K_{\ell 2}$ and $K_{\ell 3}$ decay rates can be predicted with great accuracy, providing non-trivial tests of the SM and allowing for the extraction of $V_{u s} . K_{\ell 4}$ decays are used as sensitive probes of chiral dynamics in $\pi \pi$ scattering whereas $K_{e 5}$ decays are included for completeness only.

The dominant nonleptonic decays $K \rightarrow 2 \pi, 3 \pi$ are treated in Sec. V. The two-pion modes are used to extract the leading-order (LO) nonleptonic LECs $G_{8}, G_{27}$ on the basis of a next-to-leading-order (NLO) calculation. To determine the s-wave $\pi \pi$ phase shift difference $\delta_{0}\left(M_{K}\right)-\delta_{2}\left(M_{K}\right)$, inclusion of isospin-violating corrections is mandatory. This applies also to the SM prediction of the CP-violating ratio $\epsilon^{\prime} / \epsilon$ although the theoretical precision still does not match the experimental accuracy. For the three-pion modes, NLO corrections significantly improve the agreement between theory and experiment. In addition to the CP-violating decay $K_{S} \rightarrow 3 \pi^{0}$, special attention has been given to CP-violating asymmetries in the linear Dalitz plot parameter for the threepion decays of charged kaons. Somewhat unexpectedly, $K \rightarrow 3 \pi$ decays with at least two $\pi^{0}$ in the final state allow for a precise extraction of s-wave $\pi \pi$ scattering lengths by analyzing the cusp near threshold.

Rare and radiative decays are considered in Sec.VI. We first summarize the status of the rare decays $K_{L} \rightarrow \pi^{0} \nu \bar{\nu}$ and $K^{ \pm} \rightarrow \pi^{ \pm} \nu \bar{\nu}$ that can be predicted with a precision surpassing any other FCNC process involving quarks. The modes $K \rightarrow \pi \pi \nu \bar{\nu}$ can also be predicted with good accuracy but experimental limits are still far above the rates expected in the SM. Most of the remaining radiative modes $K \rightarrow \gamma^{(*)} \gamma^{(*)}, K \rightarrow \ell^{+} \ell^{-}, K \rightarrow \pi \ell^{+} \ell^{-}$, $K \rightarrow \pi \gamma \gamma^{(*)}$ and $K \rightarrow \pi \pi \gamma^{(*)}$ are dominated by longdistance dynamics to be analyzed in the CHPT framework. All channels have been calculated to NLO but in many cases estimates of the dominant next-to-nextto-leading-order (NNLO) effects are also available. Although of minor phenomenological interest at present, the decays $K^{0} \rightarrow 3 \gamma, K_{L} \rightarrow \gamma \gamma \ell^{+} \ell^{-}, K_{L} \rightarrow \gamma \nu \bar{\nu}$, $K_{S} \rightarrow \ell_{1}^{+} \ell_{1}^{-} \ell_{2}^{+} \ell_{2}^{-}, K^{0} \rightarrow \pi^{0} \pi^{0} \gamma, K^{0} \rightarrow \pi^{0} \pi^{0} \gamma \gamma$ and $K \rightarrow 3 \pi \gamma$ are included for completeness. Conclusions and an outlook are presented in Sec. VII. Some one-loop functions are collected in the appendix.

\section{THEORETICAL FRAMEWORK}

\section{A. Short-distance description}

The SM predicts strangeness-changing transitions with $\Delta S=1$ via $W$ exchange between two weak charged cur- rents. At the kaon mass scale, the heavy $W$ boson can be integrated out and the interaction is described in terms of effective four-fermion operators.

Semileptonic transitions are mediated by the effective Lagrangian

$$
\begin{aligned}
\mathcal{L}_{\mathrm{eff}}= & -\frac{G_{F}}{\sqrt{2}} S_{\mathrm{EW}}^{1 / 2}\left[\bar{\ell} \gamma_{\mu}\left(1-\gamma_{5}\right) \nu_{\ell}\right]\left[\bar{u}_{i} \gamma^{\mu}\left(1-\gamma_{5}\right) V_{i j} d_{j}\right] \\
& + \text { h.c. }
\end{aligned}
$$

where $V_{i j}$ denotes the element $i j$ of the CKM matrix Cabibbo, 1963, Kobayashi and Maskawa, 1973) and $G_{F}=1.1663788(7) \times 10^{-5} \mathrm{GeV}^{-2}$ (Webber et al. 2011 ) is the Fermi constant as extracted from muon decay. The universal short-distance factor

$$
\begin{aligned}
S_{\mathrm{EW}} & =1+\frac{2 \alpha}{\pi}\left(1-\frac{\alpha_{s}}{4 \pi}\right) \ln \frac{M_{Z}}{M_{\rho}}+\mathcal{O}\left(\frac{\alpha \alpha_{s}}{\pi^{2}}\right) \\
& =1.0223 \pm 0.0005
\end{aligned}
$$

encodes electroweak corrections not included in $G_{F}$ (Sirlin, 1978, 1982) and small QCD effects (Marciano and Sirlin, 1993).

$W$ exchange between two quark currents generates the $\Delta S=1$ four-quark operator

$$
Q_{2}=\left[\bar{s} \gamma^{\mu}\left(1-\gamma_{5}\right) u\right]\left[\bar{u} \gamma_{\mu}\left(1-\gamma_{5}\right) d\right],
$$

mediating nonleptonic $K$ decays. Gluonic corrections bring further $\Delta S=1$ operators, which mix under renormalization (Altarelli and Maiani, 1974, Gaillard and Lee, 1974a; Shifman et al. 1977; Vainshtein et al., 1975):

$$
\begin{aligned}
& Q_{1}=\left[\bar{s}^{\alpha} \gamma^{\mu}\left(1-\gamma_{5}\right) u^{\beta}\right]\left[\bar{u}^{\beta} \gamma_{\mu}\left(1-\gamma_{5}\right) d^{\alpha}\right] \\
& Q_{3}=\left[\bar{s} \gamma^{\mu}\left(1-\gamma_{5}\right) d\right] \sum_{q=u, d, s}\left[\bar{q} \gamma_{\mu}\left(1-\gamma_{5}\right) q\right] \\
& Q_{4}=\left[\bar{s}^{\alpha} \gamma^{\mu}\left(1-\gamma_{5}\right) d^{\beta}\right] \sum_{q=u, d, s}\left[\bar{q}^{\beta} \gamma_{\mu}\left(1-\gamma_{5}\right) q^{\alpha}\right], \\
& Q_{5}=\left[\bar{s} \gamma^{\mu}\left(1-\gamma_{5}\right) d\right] \sum_{q=u, d, s}\left[\bar{q} \gamma_{\mu}\left(1+\gamma_{5}\right) q\right], \\
& Q_{6}=\left[\bar{s}^{\alpha} \gamma^{\mu}\left(1-\gamma_{5}\right) d^{\beta}\right] \sum_{q=u, d, s}\left[\bar{q}^{\beta} \gamma_{\mu}\left(1+\gamma_{5}\right) q^{\alpha}\right],
\end{aligned}
$$

where $\alpha, \beta$ denote color indices and color-singlet currents are understood whenever color labels are not explicit $\left(\bar{q} \Gamma q \equiv \bar{q}^{\alpha} \Gamma q^{\alpha}\right)$.

Owing to the presence of very different mass scales $\left(M_{\pi}<M_{K} \ll M_{W}\right)$, the QCD corrections are amplified by large logarithms. The short-distance logarithmic corrections can be summed up using the OPE and the renormalization group, all the way down from $M_{W}$ to scales $\mu<m_{c}$ (Gilman and Wise, 1979, 1980). One gets in this way an effective Lagrangian, defined in the threeflavor theory (Buras, 1998,

$$
\mathcal{L}_{\mathrm{eff}}^{\Delta S=1}=-\frac{G_{F}}{\sqrt{2}} V_{u d} V_{u s}^{*} \sum_{i=1}^{13} C_{i}(\mu) Q_{i}(\mu),
$$


which is a sum of local four-fermion operators $Q_{i}$, constructed with the light degrees of freedom $(m<\mu)$, modulated by Wilson coefficients $C_{i}(\mu)$, which are functions of the heavy masses $\left(M_{Z}, M_{W}, m_{t}, m_{b}, m_{c}>\mu\right)$ and CKM parameters. The unitarity of the CKM matrix,

$$
\lambda_{u}+\lambda_{c}+\lambda_{t}=0, \quad \lambda_{q} \equiv V_{q d} V_{q s}^{*},
$$

allows to write the Wilson coefficients in the form

$$
C_{i}(\mu)=z_{i}(\mu)+\tau y_{i}(\mu),
$$

where $\tau=-\lambda_{t} / \lambda_{u}$. The CP-violating decay amplitudes are proportional to the components $y_{i}(\mu)$.

The Wilson coefficients are known at the NLO (Buras et al., 1993a, 1996, 1993b; Ciuchini et al., 1993,1994 , 1995). This includes all corrections of $\mathcal{O}\left(\alpha_{s}^{n} t^{n}\right)$ and $\mathcal{O}\left(\alpha_{s}^{n+1} t^{n}\right)$, where $t=\ln \left(M_{1} / M_{2}\right)$ refers to the logarithm of any ratio of heavy mass scales $M_{1}, M_{2} \geq \mu$. Moreover, the full $m_{t} / M_{W}$ dependence (at lowest order in $\alpha_{s}$ ) has been taken into account.

The combination $Q_{-}=Q_{2}-Q_{1}$ and the 'penguin' operators $Q_{i}, i=3,4,5,6$, induce pure $\Delta I=1 / 2$ transitions and transform like $\left(8_{L}, 1_{R}\right)$ under chiral SU(3) $\times$ $\mathrm{SU}(3)_{\mathrm{R}}$ transformations in flavor space, while $Q^{(27)}=$ $2 Q_{2}+3 Q_{1}-Q_{3}$ transforms like a $\left(27_{L}, 1_{R}\right)$ operator that induces both $\Delta I=1 / 2$ and $\Delta I=3 / 2$ transitions.

The inclusion of virtual electromagnetic interactions brings in the additional electromagnetic penguin operators (Bijnens and Wise, 1984, Buras and Gérard, 1987, Lusignoli, 1989: Sharpe, 1987)

$$
\begin{aligned}
Q_{7} & =\frac{3}{2}\left[\bar{s} \gamma^{\mu}\left(1-\gamma_{5}\right) d\right] \sum_{q=u, d, s} e_{q}\left[\bar{q} \gamma_{\mu}\left(1+\gamma_{5}\right) q\right], \\
Q_{8} & =\frac{3}{2}\left[\bar{s}^{\alpha} \gamma^{\mu}\left(1-\gamma_{5}\right) d^{\beta}\right] \sum_{q=u, d, s} e_{q}\left[\bar{q}^{\beta} \gamma_{\mu}\left(1+\gamma_{5}\right) q^{\alpha}\right], \\
Q_{9} & =\frac{3}{2}\left[\bar{s} \gamma^{\mu}\left(1-\gamma_{5}\right) d\right] \sum_{q=u, d, s} e_{q}\left[\bar{q} \gamma_{\mu}\left(1-\gamma_{5}\right) q\right], \quad(2.8) \\
Q_{10} & =\frac{3}{2}\left[\bar{s}^{\alpha} \gamma^{\mu}\left(1-\gamma_{5}\right) d^{\beta}\right] \sum_{q=u, d, s} e_{q}\left[\bar{q}^{\beta} \gamma_{\mu}\left(1-\gamma_{5}\right) q^{\alpha}\right],
\end{aligned}
$$

where $e_{q}$ denotes the corresponding quark charges in units of $e=\sqrt{4 \pi \alpha}$. Their Wilson coefficients get also higher-order electroweak contributions from $Z$-penguin and $W$-box diagrams. Under the action of the chiral group $\mathrm{SU}(3)_{\mathrm{L}} \times \mathrm{SU}(3)_{\mathrm{R}}$ the operators $Q_{7}$ and $Q_{8}$ transform like combinations of $\left(8_{L}, 1_{R}\right)$ and $\left(8_{L}, 8_{R}\right)$ operators, while $Q_{9}$ and $Q_{10}$ transform like combinations of $\left(8_{L}, 1_{R}\right)$ and $\left(27_{L}, 1_{R}\right)$.

Three more operators need to be considered in processes with leptons in the final state (Buchalla et al.

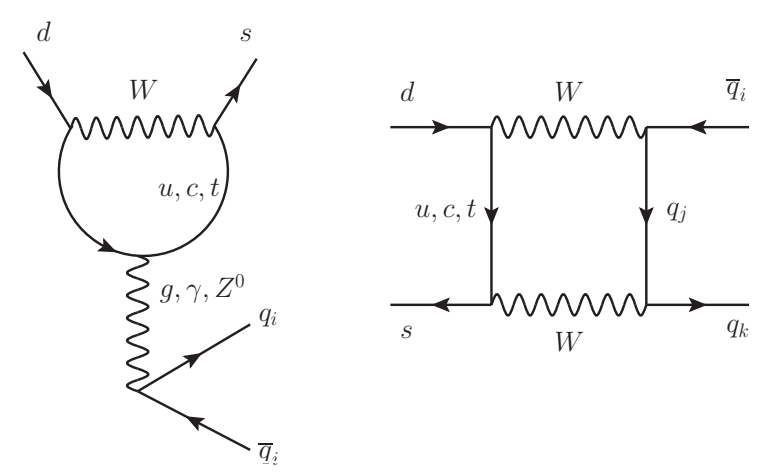

FIG. 1 Penguin and box topologies.

1996: Gilman and Wise, 1980):

$$
\begin{aligned}
Q_{11} & \equiv Q_{7 V}=\left[\bar{s} \gamma^{\mu}\left(1-\gamma_{5}\right) d\right] \sum_{\ell=e, \mu}\left[\bar{\ell} \gamma_{\mu} \ell\right], \\
Q_{12} \equiv Q_{7 A} & =\left[\bar{s} \gamma^{\mu}\left(1-\gamma_{5}\right) d\right] \sum_{\ell=e, \mu}\left[\bar{\ell} \gamma_{\mu} \gamma_{5} \ell\right], \\
Q_{13} \equiv Q(\bar{\nu} \nu) & =\left[\bar{s} \gamma^{\mu}\left(1-\gamma_{5}\right) d\right]\left[\bar{\nu} \gamma_{\mu}\left(1-\gamma_{5}\right) \nu\right] .
\end{aligned}
$$

The mixing between the $K^{0}$ and its antiparticle is induced through box diagrams with two $W$ exchanges (Gaillard and Lee, 1974b), which in the three-flavor theory generate the effective Lagrangian (Gilman and Wise, 1983)

$$
\mathcal{L}_{\text {eff }}^{\Delta S=2}=-\frac{G_{F}^{2} M_{W}^{2}}{(4 \pi)^{2}} C_{\Delta S=2}(\mu) Q_{\Delta S=2}(\mu),
$$

with

$$
Q_{\Delta S=2}=\left[\bar{s} \gamma^{\mu}\left(1-\gamma_{5}\right) d\right]\left[\bar{s} \gamma_{\mu}\left(1-\gamma_{5}\right) d\right] .
$$

There are in addition long-distance contributions from two $\mathcal{L}_{\text {eff }}^{\Delta S=1}$ insertions.

The box and penguin topologies, shown in Fig. 1] introduce contributions proportional to $\lambda_{q}(q=u, c, t)$ from virtual up-type quark exchanges. The hierarchy of CKM factors is better visualized through the Wolfenstein (1983) parametrization: $\lambda_{u} \sim \lambda \equiv\left|V_{u s}\right|, \lambda_{c} \sim-\lambda$ and $\overline{\lambda_{t}} \sim-A^{2} \lambda^{5}$, where $A=\left|V_{c b}\right| / \lambda^{2}$. The sum of the three contributions cancels for equal up-type quark masses, owing to the unitarity relation (2.6). The quark-mass dependence of the associated loop functions breaks the GIM cancellation and strongly enhances the relevance of the top contribution in short-distance dominated processes. This is especially true for CP-violating effects, which are proportional to (Buras et al., 1994b)

$$
\operatorname{Im}\left(\lambda_{t}\right)=-\operatorname{Im}\left(\lambda_{c}\right) \approx \eta \lambda^{5} A^{2} .
$$

The corresponding CP-conserving factors are given to a very good approximation by

$$
\begin{aligned}
& \operatorname{Re}\left(\lambda_{c}\right)=-\lambda\left(1-\frac{\lambda^{2}}{2}\right) \\
& \operatorname{Re}\left(\lambda_{t}\right)=-A^{2} \lambda^{5}\left(1-\frac{\lambda^{2}}{2}\right)(1-\bar{\rho}) .
\end{aligned}
$$


The value of $\lambda$ can be extracted from $K_{\ell 3}$ decays and is given in Eq. (4.58). The remaining CKM parameters are obtained from global SM fits to flavor-changing data (Bona et al., 2006; Charles et al. 2005). We will adopt the values (Charles et al. 2010)

$$
\begin{aligned}
& A=0.812_{-0.027}^{+0.013}, \quad \bar{\rho}=0.144 \pm 0.025, \\
& \bar{\eta} \equiv\left(1-\frac{\lambda^{2}}{2}\right) \eta=0.342 \pm 0.016
\end{aligned}
$$

In order to fully calculate the kaon decay amplitudes, we also need to know the matrix elements of the operators $Q_{i}$ between the initial and final states, which involve nonperturbative dynamics at low energies. These hadronic matrix elements should cancel the renormalization-scale dependence of the Wilson coefficients $C_{i}(\mu)$. Methods to tackle this challenging task include lattice gauge theory (Boucaud et al., 2005 , Boyle and Garron, 2010: Colangelo et al. || 2011;|Goode and Lightman,| 2010; Kim and Sachrajda | 2010; Laiho and Van de Water |2010| Liu, 2010), the $1 / N_{C}$ expansion (Bardeen et al. $, 1987 \mathrm{a} \mid \mathrm{b}, 1988$, Hambye et al., 2000, 1998, 1999), QCD sum rules (Guberina et al. | 1985: Jamin and Pich, 1994; : Pich et al., 1986||Pich and de Rafael, 1985, 1987, 1996, Prades et al., 1991), functional bosonization techniques (Bruno and Prades, 1993 Friot et al., 2004a; Hambye et al. , 2003 Knecht et al., 1999b, 2001; Peris and de Rafael, 2000; Pich and de Rafael 1991) and dynamical models (Antonelli et al. 1996a b, 1997; Bertolini et al., 1995, 1998: Bijnens et al., 2001, 2006 |Bijnens and Prades, 1995a b, 1999, 2000a b).

CHPT provides a solid effective field theory framework to analyze systematically the long-distance dynamics, which we discuss next. Nevertheless, non-perturbative techniques remain necessary for matching the effective low-energy theory with the underlying QCD Lagrangian.

\section{B. Chiral perturbation theory}

Most kaon decays are governed by physics at long distances. Below the resonance region one can use symmetry considerations to define another effective field theory in terms of the QCD Goldstone bosons. CHPT describes (Gasser and Leutwyler, 1985a; Weinberg, 1979) the pseudoscalar-octet dynamics through a perturbative expansion in powers of momenta and quark masses over the chiral symmetry-breaking scale $\Lambda_{\chi} \sim 1 \mathrm{GeV}$. Chiral symmetry fixes the allowed operators, providing a comprehensive framework for both semileptonic and nonleptonic kaon decays including radiative corrections. For general reviews of CHPT, see Ecker (1995), Pich (1995), de Rafael (1995), Pich (1998), Scherer (2003), Gasser (2004) and Bijnens (2007). The standard textbook on the SM at low energies is Donoghue et al. (1992).

The effective mesonic chiral Lagrangian in use today is listed schematically in Table I] The strong chiral Lagrangian relevant for (semi)leptonic decays to NNLO is
TABLE I The $\Delta S=1$ chiral Lagrangian describing (semi)leptonic and nonleptonic kaon decays. Leptons are incorporated for radiative corrections in (semi)leptonic decays. The numbers in brackets denote the number of LECs.

\begin{tabular}{|c|c|}
\hline $\mathcal{L}_{\text {chiral order }}(\#$ of LECs $)$ & loop order \\
\hline $\mathcal{L}_{p^{2}}(2)+\mathcal{L}_{p^{4}}^{\text {odd }}(0)+\mathcal{L}_{G_{8} p^{2}}^{\Delta S=1}(1)+\mathcal{L}_{G_{27} p^{2}}^{\Delta S}(1)$ & $L=0$ \\
$+\mathcal{L}_{G_{8} e^{2} p^{0}}^{\mathrm{emweak}}(1)+\mathcal{L}_{e^{2} p^{0}}^{\mathrm{em}}(1)+\mathcal{L}_{\mathrm{kin}}^{\text {lepton }}(0)$ & \\
$+\mathcal{L}_{p^{4}}(10)+\mathcal{L}_{p^{6}}^{\text {odd }}(23)+\mathcal{L}_{G_{8} p^{4}}^{\Delta S=1}(22)$ & $L \leq 1$ \\
$+\mathcal{L}_{G_{27} p^{4}}^{\Delta S}(28)+\mathcal{L}_{G_{8} e^{2} p^{2}}^{\mathrm{emweak}}(14)$ & \\
$+\mathcal{L}_{e^{2} p^{2}}^{\mathrm{em}}(13)+\mathcal{L}_{e^{2} p^{2}}^{\text {lepton }}(5)$ & \\
$+\mathcal{L}_{p^{6}}(90)$ & $L \leq 2$ \\
\hline
\end{tabular}

given by

$$
\begin{aligned}
\mathcal{L}_{\text {strong }}= & \underbrace{\frac{F_{0}^{2}}{4}\left\langle D_{\mu} U D^{\mu} U^{\dagger}+\chi U^{\dagger}+\chi^{\dagger} U\right\rangle}_{\mathcal{L}_{p^{2}}(2)} \\
& +\underbrace{\sum_{i} L_{i} O_{i}^{p^{4}}}_{\mathcal{L}_{p^{4}}(10)}+\underbrace{\mathcal{L}_{\mathrm{WZW}}}_{\mathcal{L}_{p^{4}}^{\text {odd }}(0)} \\
& +\underbrace{\sum_{i} C_{i} O_{i}^{p^{6}}}_{\mathcal{L}_{p^{6}}(90)}+\underbrace{\sum_{i} C_{i}^{W} O_{i}^{p^{6}, \text { odd }}}_{\mathcal{L}_{p^{6}}^{\text {odd }}(23)}
\end{aligned}
$$

$F_{0}$ is the pion decay constant in the limit of chiral SU(3), the $\mathrm{SU}(3)$ matrix field $U$ contains the pseudoscalar fields, $D_{\mu}$ is the covariant derivative in the presence of external vector and axial-vector fields, the scalar field $\chi$ accounts for explicit chiral symmetry breaking through the quark masses $m_{u}, m_{d}, m_{s}$, and $\langle\ldots\rangle$ stands for the threedimensional flavor trace. Explicit forms of the higherorder Lagrangians can be found in Gasser and Leutwyler (1985a), Wess and Zumino (1971), Witten (1983), Bijnens et al. (1999, 2002) and Ebertshauser et al. (2002).

The nonleptonic weak Lagrangian to NLO is given by

$$
\begin{aligned}
\mathcal{L}_{\text {weak }}= & \underbrace{G_{8} F_{0}^{4}\left\langle\lambda D^{\mu} U^{\dagger} D_{\mu} U\right\rangle}_{\mathcal{L}_{G_{8} p^{2}}^{\Delta S^{2}}(1)} \\
& +\underbrace{G_{27} F_{0}^{4}\left(L_{\mu 23} L_{11}^{\mu}+\frac{2}{3} L_{\mu 21} L_{13}^{\mu}\right)}_{\mathcal{L}_{G_{27} p^{2}}^{\Delta S}(1)} \\
& +\underbrace{G_{8} F_{0}^{2} \sum_{i} N_{i} O_{i}^{8}}_{\mathcal{L}_{G_{8} p^{4}}^{\Delta S}(22)}+\underbrace{G_{27} F_{0}^{2} \sum_{i} D_{i} O_{i}^{27}}_{\mathcal{L}_{G_{27} p^{4}}^{\Delta S}(28)}
\end{aligned}
$$


The matrix $L_{\mu}=i U^{\dagger} D_{\mu} U$ represents the octet of $V-A$ currents to lowest order in derivatives; $\lambda=\left(\lambda_{6}-i \lambda_{7}\right) / 2$ projects onto the $\bar{s} \rightarrow \bar{d}$ transition. The terms proportional to $G_{8}$ and $G_{27}$ transform under chiral transformations like $\left(8_{L}, 1_{R}\right)$ and $\left(27_{L}, 1_{R}\right)$, respectively, providing the most general effective realization of the corresponding short-distance operators in Eq. (2.5). The Lagrangians can be found in Cronin (1967), Kambor et al. (1990) and Ecker et al. (1993).

To include electromagnetic corrections for both (semi)leptonic and nonleptonic decays, we also need the chiral Lagrangians

$$
\begin{aligned}
\mathcal{L}_{\text {lepton }} & =\mathcal{L}_{\text {kin }}^{\text {lepton }}(0)+\underbrace{e^{2} F_{0}^{2} \sum_{i} X_{i} O_{i}^{\text {lepton }}}_{\mathcal{L}_{e^{2} p^{2}}^{\text {lepton }}(5)}, \\
\mathcal{L}_{\text {elm }}= & \underbrace{e^{2} Z F_{0}^{4}\left\langle Q U^{\dagger} Q U\right\rangle}_{\mathcal{L}_{e^{2} p^{0}}^{\text {em }}(1)}+\underbrace{e^{2} G_{8} g_{\text {ewk }} F_{0}^{6}\left\langle\lambda U^{\dagger} Q U\right\rangle}_{\mathcal{L}_{G_{8} e^{2} p^{0}}^{\text {emwea }}(1)} \\
& +\underbrace{e^{2} F_{0}^{2} \sum_{i}^{2} K_{i} O_{i}^{e^{2} p^{2}}}_{\mathcal{L}_{e^{2} p^{2}}^{\text {em }}} \\
& +\underbrace{e^{2} G_{8} F_{0}^{4} \sum_{i} Z_{i} O_{i}^{E W}}_{\mathcal{L}_{G_{8} e^{2} p^{2}}^{\text {emweak }}(14)}+\text { h.c. },
\end{aligned}
$$

where $Q$ is the diagonal matrix of quark charges. The corresponding Lagrangians can be found in Ecker et al. (1989b), Bijnens and Wise (1984), Grinstein et al. (1986), Urech (1995), Ecker et al. (2000a) and Knecht et al. (2000). The terms proportional to $g_{\text {ewk }}$ and $Z_{i}$ provide the low-energy realization of the electromagnetic penguin operators 2.8), while those with couplings $X_{i}$ account for the operators in Eq. 2.9 with explicit lepton fields.

The chiral realization of the $\Delta S=2$ effective Lagrangian contains a single $\mathcal{O}\left(p^{2}\right)$ operator:

$$
\mathcal{L}_{\text {eff }}^{\Delta S=2}=\frac{G_{F}^{2} M_{W}^{2}}{(4 \pi)^{2}} g_{\Delta S=2} F_{0}^{4}\left\langle\lambda U^{\dagger} D^{\mu} U\right\rangle\left\langle\lambda U^{\dagger} D_{\mu} U\right\rangle .
$$

The $\mathcal{O}\left(p^{4}\right)$ operators were discussed by Kambor et al. (1990) and Esposito-Farèse (1991).

The CHPT framework determines the $K$ decay amplitudes in terms of the LECs multiplying the relevant operators in the chiral Lagrangian. These LECs encode all information about the short-distance dynamics, while the chiral operators yield the most general form of the low-energy amplitudes compatible with chiral symmetry. Chiral loops generate non-polynomial contributions, with logarithms and threshold factors as required by unitarity.

Practically all kaon decays discussed in this review have been calculated at least up to $\mathcal{O}\left(p^{4}\right)$. The strong and nonleptonic parts of the corresponding NLO amplitudes can be given in compact form in terms of the generating functional. The matrix elements and form factors with at most six external particles (an external photon counts as two particles), at most one $W$ (semileptonic decays) and with at most three propagators in the one-loop amplitudes were presented in closed form by Unterdorfer and Ecker (2005). This includes most of the processes covered in this review. Except for electromagnetic and anomalous contributions, all amplitudes to $\mathcal{O}\left(p^{4}\right)$ can be obtained from a Mathematica program written by René Unterdorfer. The code along with several examples can be downloaded from http://homepage.univie.ac.at/Gerhard.Ecker/CPTamp.html.

\section{ESTIMATES OF LOW-ENERGY CONSTANTS}

A first-principle calculation of LECs requires to perform the matching between CHPT and the underlying SM. This is a very difficult task. In many cases one resorts to phenomenology to determine the values of the LECs. For instance, most of the $\mathcal{O}\left(p^{4}\right)$ couplings of the strong chiral Lagrangian, $L_{i}$, are rather well known (Bijnens and Jemos, 2011; Ecker, 2007) from low-energy data ( $\pi \pi$ scattering, $\pi$ and $K$ decay constants and masses, $\pi$ electromagnetic radius, $\pi \rightarrow e \nu \gamma, \tau$ decay). The electromagnetic LEC $Z$ arising at $\mathcal{O}\left(e^{2} p^{0}\right)$ can be expressed in terms of the squared-mass difference of the pions:

$$
M_{\pi^{ \pm}}^{2}-M_{\pi^{0}}^{2}=8 \pi \alpha Z F_{0}^{2} .
$$

The matching procedure is of course simpler whenever the hadronic dynamics gets reduced to quark currents. For instance, in the CHPT language, the short-distance enhancement of semileptonic decays is encoded in the factor (Descotes-Genon and Moussallam, 2005, Knecht et al. 2000)

$$
1-\frac{e^{2}}{2}\left(X_{6}^{r}-4 K_{12}^{r}\right) \equiv 1-\frac{e^{2}}{2} X_{6}^{\text {phys }},
$$

which is related to $S_{\mathrm{EW}}$ in the Lagrangian 2.1 by

$$
e^{2} X_{6}^{\text {phys }}\left(M_{\rho}\right)=S_{\mathrm{EW}}-1+e^{2} \tilde{X}_{6}^{\text {phys }}\left(M_{\rho}\right),
$$

where $\tilde{X}_{6}^{\text {phys }}\left(M_{\rho}\right)$ denotes a residual long-distance contribution (Descotes-Genon and Moussallam, 2005).

\section{A. The large- $\mathrm{N}_{\mathrm{C}}$ limit of $\mathrm{QCD}$}

The limit of an infinite number of quark colors is a very useful starting point to understand many features of QCD ('t Hooft, 1974a b; Witten, 1979). Assuming confinement, the strong dynamics at $N_{C} \rightarrow \infty$ is given by tree diagrams with infinite sums of hadron exchanges, which correspond to the tree approximation for some local effective Lagrangian. Hadronic loops generate corrections suppressed by factors of $1 / N_{C}$. Resonance chiral 
theory (Cirigliano et al., 2006, Ecker et al., 1989a b) provides an appropriate framework to incorporate the massive mesonic states (Pich, 2002). Integrating out the resonance fields, one recovers the usual CHPT Lagrangian with explicit values for the LECs, parametrized in terms of resonance masses and couplings. The resonance chiral theory generates Green functions that interpolate between QCD and CHPT. Analyzing these Green functions, both for large and small momenta, one gets QCD constraints on the resonance couplings and, therefore, information on the LECs.

Truncating the infinite tower of meson resonances to the lowest states with $0^{-+}, 0^{++}, 1^{--}$and $1^{++}$quantum numbers, one gets a very successful prediction of the $\mathcal{O}\left(p^{4} N_{C}\right)$ strong CHPT couplings in terms of only three parameters: $M_{V}, M_{S}$ and $F_{0}$. This provides a theoretical understanding of the role of resonance saturation in low-energy phenomenology, which was recently extended to $\mathcal{O}\left(p^{6}\right)$ (Cirigliano et al. 2006). Of particular interest for our present purposes are the resulting predictions for the $\mathcal{O}\left(p^{6}\right)$ couplings $C_{12}$ and $C_{34}$, which govern the amount of $\mathrm{SU}(3)$ breaking in the $K_{\ell 3}$ form factor at zero momentum transfer (Cirigliano et al., 2005, 2006):

$$
C_{12}=-\frac{F_{0}^{2}}{8 M_{S}^{4}}, \quad C_{34}=\frac{3 F_{0}^{2}}{16 M_{S}^{4}}+\frac{F_{0}^{2}}{16}\left(\frac{1}{M_{S}^{2}}-\frac{1}{M_{P}^{2}}\right)^{2}
$$

The large- $N_{C}$ limit turns out to be very useful to analyze the nonleptonic weak Lagrangian, because the Tproduct of two color-singlet quark currents factorizes:

$$
\langle J \cdot J\rangle=\langle J\rangle\langle J\rangle\left\{1+\mathcal{O}\left(\frac{1}{N_{C}}\right)\right\} .
$$

Since quark currents have well-known CHPT realizations, the hadronization of the short-distance operators $Q_{i}$ can then be done in a straightforward way. As a result, in this large- $N_{C}$ framework the electroweak chiral couplings can be related to strong and electromagnetic LECs of order $p^{2}, p^{4}, p^{6}$ and $e^{2} p^{2}$, respectively. The lowest-order electroweak LECs take the following values at large $N_{C}$ (Cirigliano et al. 2004a; Pallante et al., 2001):

$$
\begin{aligned}
g_{8}^{\infty}= & -\frac{2}{5} C_{1}(\mu)+\frac{3}{5} C_{2}(\mu)+C_{4}(\mu)-16 L_{5} B(\mu) C_{6}(\mu) \\
g_{27}^{\infty}=\frac{3}{5}\left[C_{1}(\mu)\right. & \left.+C_{2}(\mu)\right] \\
\left(e^{2} g_{8} g_{\mathrm{ewk}}\right)^{\infty}= & -3 B(\mu) C_{8}(\mu) \\
& -\frac{16}{3} C_{6}(\mu) e^{2}\left(K_{9}-2 K_{10}\right)
\end{aligned}
$$

and

$$
g_{\Delta S=2}^{\infty}=C_{\Delta S=2}(\mu),
$$

where the dimensionless couplings $g_{8}, g_{27}$ are defined as

$$
G_{8,27}=-\frac{G_{F}}{\sqrt{2}} V_{u d} V_{u s}^{*} g_{8,27}
$$

The operators $Q_{i}(i \neq 6,8)$ factorize into products of left- and right-handed vector currents, which are renormalization-invariant quantities. Thus, the large- $N_{C}$ factorization of these operators does not generate any scale dependence. The only anomalous dimensions that survive when $N_{C} \rightarrow \infty$ are the ones corresponding to $Q_{6}$ and $Q_{8}$ (Bardeen et al. 1987b; Buras and Gérard, 1987). These operators factorize into color-singlet scalar and pseudoscalar currents, which are $\mu$ dependent. The CHPT evaluation of the scalar and pseudoscalar currents provides, of course, the right $\mu$ dependence, since only physical observables can be realized in the low-energy theory. What one actually finds is the chiral realization of the renormalization-invariant products $m_{q} \bar{q}\left(1, \gamma_{5}\right) q$. This generates in Eq. (3.6) the factors

$$
\begin{array}{r}
B(\mu)=\left[\frac{M_{K}^{2}}{\left(m_{s}+m_{d}\right)(\mu) F_{\pi}}\right]^{2} \times\left\{1+\frac{8 M_{\pi}^{2}}{F_{\pi}^{2}} L_{5}\right. \\
\left.-\frac{16 M_{K}^{2}}{F_{\pi}^{2}}\left(2 L_{8}-L_{5}\right)+8 \frac{2 M_{K}^{2}+M_{\pi}^{2}}{F_{\pi}^{2}}\left(3 L_{4}-4 L_{6}\right)\right\},
\end{array}
$$

which exactly cancel the $\mu$ dependence of $C_{6,8}(\mu)$ at large $N_{C}$. There remains a dependence at NLO.

The large- $N_{C}$ expressions imply the numerical values

$$
\begin{aligned}
& g_{8}^{\infty}=\left(1.13 \pm 0.05_{\mu} \pm 0.08_{L_{5}} \pm 0.05_{m_{s}}\right) \\
& +\tau\left(0.64 \pm 0.15_{\mu} \pm 0.20_{L_{5}-0.16}+0.25 m_{s}\right), \\
& g_{27}^{\infty}=0.46 \pm 0.01_{\mu}, \\
& \left(g_{8} g_{\text {ewk }}\right)^{\infty}=\left(-1.60 \pm 0.86_{\mu} \pm 0.25_{K_{i}-0.35 m_{s}}+0.57\right. \\
& -\tau\left(25.0 \pm 4.5_{\mu} \pm 1.0_{K_{i}-5.6}+9.1\right),
\end{aligned}
$$

where $\tau$ is the ratio of CKM matrix elements in Eq. (2.7) and the main sources of uncertainty are indicated.

While the CP-even part of $g_{8}^{\infty}$ gets contributions from all $\left(8_{L}, 1_{R}\right)$ short-distance operators, its CP-odd component is completely dominated by the strong penguin contribution, proportional to $\tau y_{6}(\mu)$. The CP-odd component of $g_{\text {ewk }}$ is dominated by the electroweak penguin contribution, proportional to $\tau y_{8}(\mu)$, while the CPeven part receives contributions of similar size from both strong $\left(Q_{6}\right)$ and electroweak $\left(Q_{8}\right)$ penguin operators. Explicit predictions for the higher-order electroweak LECs can be found in Cirigliano et al. (2004a).

It is important to stress that the large- $N_{C}$ limit is only applied to the matching between the three-flavor quark theory and CHPT. The evolution from the electroweak scale down to $\mu<m_{c}$ has to be done without any unnecessary expansion in powers of $1 / N_{C}$; otherwise, one would miss large corrections of the form $\ln (M / m) / N_{C}$, with $M \gg m$ two widely separated scales. Similarly, the long-distance rescattering of the final pions in the $K$ decay generates large logarithmic corrections through chiral loops, which are of higher order in both the momentum and $1 / N_{C}$ expansions (Pallante et al. 2001). These next-to-leading contributions, which give rise to the large s-wave $\pi \pi$ strong phases, are rigorously incorporated through the CHPT framework. 


\section{B. Lattice determinations}

Lattice results for strong LO and NLO LECs, both for $\mathrm{SU}(2)$ and for SU(3), have recently been summarized by the Flavianet Lattice Averaging Group -FLAG- (Colangelo et al. 2011). We refer to this comprehensive review for a detailed discussion.

\section{LEPTONIC AND SEMILEPTONIC DECAYS}

Purely leptonic and semileptonic modes are among the theoretically cleanest $K$ decays. Using CHPT and lattice QCD, $K_{\ell 2}$ and $K_{\ell 3}$ decay rates can be predicted with high accuracy and provide non-trivial tests of the SM and its extensions. On the other hand, $K_{\ell 4}$ decays can be used as probes of chiral dynamics in $\pi \pi$ scattering. In this section we review the current status of $K \rightarrow \ell \nu$ $\left(K_{\ell 2}\right), K \rightarrow \pi \ell \nu\left(K_{\ell 3}\right)$, and $K \rightarrow \pi \pi \ell \nu\left(K_{\ell 4}\right)$ decays, as well as their radiative counterparts.

\section{A. $K_{\ell 2}\left(\right.$ and $\left.\pi_{\ell 2}\right)$ decays}

\section{Electromagnetic corrections}

The discussion of electromagnetic contributions to $K_{\ell 2}$ decays serves as the simplest example of the treatment of electromagnetism in (semi)leptonic processes. We start with the parametrization of the inclusive $P \rightarrow \ell \nu_{\ell}(\gamma)$ decay rate proposed by Cirigliano and Rosell (2007a b) (here $P=K^{ \pm}, \pi^{ \pm}$),

$$
\begin{aligned}
\Gamma_{P_{\ell 2(\gamma)}} & =\Gamma_{P_{\ell 2}}^{(0)} S_{\mathrm{EW}}\left\{1+\frac{\alpha}{\pi} F\left(m_{\ell} / M_{P}\right)\right\} \\
\times & \left\{1-\frac{\alpha}{\pi}\left[\frac{3}{2} \ln \frac{M_{\rho}}{M_{P}}+c_{1}^{(P)}-\frac{M_{P}^{2}}{M_{\rho}^{2}} \tilde{c}_{2}^{(P)} \ln \frac{M_{\rho}^{2}}{m_{\ell}^{2}}\right.\right. \\
& \left.\left.+\frac{m_{\ell}^{2}}{M_{\rho}^{2}}\left(c_{2}^{(P)} \ln \frac{M_{\rho}^{2}}{m_{\ell}^{2}}+c_{3}^{(P)}+c_{4}^{(P)}\left(m_{\ell} / M_{P}\right)\right)\right]\right\},
\end{aligned}
$$

which is a modified version of the expression given in Marciano and Sirlin (1993). The decay rate in the absence of radiative corrections is given by

$$
\Gamma_{P_{\ell 2}}^{(0)}=\frac{G_{F}^{2}\left|V_{P}\right|^{2} F_{P}^{2}}{4 \pi} M_{P} m_{\ell}^{2}\left(1-\frac{m_{\ell}^{2}}{M_{P}^{2}}\right)^{2},
$$

where $V_{\pi}=V_{u d}, V_{K}=V_{u s}$ and $F_{\pi^{ \pm}}, F_{K^{ \pm}}$denote the pseudoscalar decay constants in pure QCD including strong isospin breaking. ${ }^{1}$ The first term in curly brackets is the universal long-distance correction for a pointlike meson. The explicit form of the one-loop function

1 For a discussion of subtleties involved in this separation, see Gasser and Zarnauskas (2010).
$F(x)$ can be found in Marciano and Sirlin (1993). The structure-dependent coefficients $c_{1}^{(P)}$ are independent of the lepton mass $m_{\ell}$ and start at $\mathcal{O}\left(e^{2} p^{2}\right)$ in CHPT. The other coefficients appear only at higher orders in the chiral expansion. The one-loop result (order $e^{2} p^{2}$ ) for $c_{1}^{(P)}$ was given by Knecht et al. (2000),

$$
\begin{aligned}
& c_{1}^{(\pi)}=-\tilde{E}^{r}\left(M_{\rho}\right)+\frac{Z}{4}\left(3+2 \ln \frac{M_{\pi}^{2}}{M_{\rho}^{2}}+\ln \frac{M_{K}^{2}}{M_{\rho}^{2}}\right), \\
& c_{1}^{(K)}=-\tilde{E}^{r}\left(M_{\rho}\right)+\frac{Z}{4}\left(3+2 \ln \frac{M_{K}^{2}}{M_{\rho}^{2}}+\ln \frac{M_{\pi}^{2}}{M_{\rho}^{2}}\right),
\end{aligned}
$$

where $Z$ is the $\mathcal{O}\left(e^{2} p^{0}\right)$ electromagnetic coupling given in Eqs. 2.18 and 3.1). $\tilde{E}^{r}\left(M_{\rho}\right)$ is a certain linear combination of LECs appearing in the Lagrangians $\mathcal{L}_{e^{2} p^{2}}^{\mathrm{em}}$ and $\mathcal{L}_{e^{2} p^{2}}^{\text {lepton }}$

$$
\begin{aligned}
\tilde{E}^{r}=\frac{1}{2}+4 \pi^{2} & \left(\frac{8}{3} K_{1}^{r}+\frac{8}{3} K_{2}^{r}+\frac{20}{9} K_{5}^{r}+\frac{20}{9} K_{6}^{r}\right. \\
& \left.-\frac{4}{3} X_{1}^{r}-4 X_{2}^{r}+4 X_{3}^{r}-\tilde{X}_{6}^{\text {phys }}\right)
\end{aligned}
$$

The results in Eq. 4.3 are a nice example demonstrating the power of effective field theory methods. We see how the electromagnetic corrections to $\pi_{\ell 2}$ and $K_{\ell 2}$ of $\mathcal{O}\left(e^{2} p^{2}\right)$ are related. In particular, $c_{1}^{(\pi)}$ and $c_{1}^{(K)}$ contain the same combination of LECs $\tilde{E}^{r}$. Taking the ratio $\Gamma_{K_{\ell 2(\gamma)}} / \Gamma_{\pi_{\ell 2(\gamma)}}$, the coupling constant $\tilde{E}^{r}$ cancels and the remaining expression

$$
c_{1}^{(K)}-c_{1}^{(\pi)}=\frac{Z}{4} \ln \frac{M_{K}^{2}}{M_{\pi}^{2}}
$$

is uniquely determined in terms of measurable quantities (Knecht et al., 2000).

Finally, we note that using the matching calculation of Descotes-Genon and Moussallam (2005) for the LEC combination $\vec{E}^{r}\left(M_{\rho}\right)$, one obtains

$$
c_{1}^{(\pi)}=-2.4 \pm 0.5, \quad c_{1}^{(K)}=-1.9 \pm 0.5,
$$

where the errors given here are based on naive power counting of unknown contributions arising at $\mathcal{O}\left(e^{2} p^{4}\right)$.

\section{Extraction of $V_{u s} / V_{u d}$}

As suggested by Marciano (2004), a determination of $\left|V_{u s} / V_{u d}\right|$ can be obtained by combining the experimental values for the decay rates $K \rightarrow \mu \nu(\gamma)$ and $\pi \rightarrow \mu \nu(\gamma)$ with the lattice determination of $F_{K} / F_{\pi}$, which is currently performed in the isospin limit $\left(m_{u}=m_{d}\right)$ of QCD. The relation to be used to extract $\left|V_{u s} / V_{u d}\right|$ reads

$$
\begin{aligned}
\frac{\Gamma_{K_{\ell 2(\gamma)}}}{\Gamma_{\pi_{\ell 2(\gamma)}}}= & \frac{\left|V_{u s}\right|^{2}}{\left|V_{u d}\right|^{2}} \frac{F_{K}^{2}}{F_{\pi}^{2}} \frac{M_{K^{ \pm}}\left(1-m_{\ell}^{2} / M_{K^{ \pm}}^{2}\right)^{2}}{M_{\pi^{ \pm}}\left(1-m_{\ell}^{2} / M_{\pi^{ \pm}}^{2}\right)^{2}} \\
& \times\left(1+\delta_{\mathrm{EM}}+\delta_{\mathrm{SU}(2)}\right),
\end{aligned}
$$


where $F_{\pi}$ and $F_{K}$ denote the decay constants in the isospin limit, $\delta_{\mathrm{EM}}$ is the long-distance electromagnetic correction (the short-distance part cancels in the ratio) and the strong isospin-breaking correction $\delta_{\mathrm{SU}(2)}$ is defined by

$$
\frac{F_{K^{ \pm}}^{2}}{F_{\pi^{ \pm}}^{2}}=\frac{F_{K}^{2}}{F_{\pi}^{2}}\left(1+\delta_{\mathrm{SU}(2)}\right) .
$$

The electromagnetic correction is given by (Cirigliano and Neufeld, 2011, Knecht et al., 2000)

$$
\begin{aligned}
\delta_{\mathrm{EM}} & =\frac{\alpha}{\pi}\left(F\left(m_{\ell} / M_{K}\right)-F\left(m_{\ell} / M_{\pi}\right)+\frac{3-Z}{4} \ln \frac{M_{K}^{2}}{M_{\pi}^{2}}\right) \\
& =-0.0069 \pm 0.0017 .
\end{aligned}
$$

The $25 \%$ uncertainty of the numerical value is an estimate of corrections arising to higher order in the chiral expansion. The correction parameter $\delta_{\mathrm{SU}(2)}$ reads (Cirigliano and Neufeld, 2011)

$$
\begin{aligned}
\delta_{\mathrm{SU}(2)}= & \sqrt{3} \varepsilon^{(2)}\left[-\frac{4}{3}\left(F_{K} / F_{\pi}-1\right)\right. \\
& \left.+\frac{1}{3(4 \pi)^{2} F_{0}^{2}}\left(M_{K}^{2}-M_{\pi}^{2}-M_{\pi}^{2} \ln \frac{M_{K}^{2}}{M_{\pi}^{2}}\right)\right],
\end{aligned}
$$

where

$$
\varepsilon^{(2)}=\frac{\sqrt{3}}{4 R}, \quad R=\frac{m_{s}-\widehat{m}}{m_{d}-m_{u}}, \quad \widehat{m}=\frac{m_{u}+m_{d}}{2} .
$$

With the FLAG (Colangelo et al. 2011$)$ averages of lattice calculations with $N_{f}=2+1$ dynamical fermions, ${ }^{2}$

$$
R=36.6 \pm 3.8, \quad F_{K} / F_{\pi}=1.193 \pm 0.006,
$$

Cirigliano and Neufeld (2011) obtained

$$
\delta_{\mathrm{SU}(2)}=-0.0044 \pm 0.0005 \pm 0.0011_{\text {higher orders }},
$$

where the uncertainty due to higher-order corrections in the chiral expansion was estimated to be at a level of $25 \%$. We note that the strong isospin-breaking correction $\delta_{\mathrm{SU}(2)}$ is of the same order of magnitude as the electromagnetic correction $\delta_{\mathrm{EM}}$ in Eq. 4.9 and should not be neglected in the extraction of the ratio $\left|V_{u s} / V_{u d}\right|$.

Combined with the measured values for the leptonic widths of the pion (Nakamura et al. 2010)

$$
\Gamma_{\pi_{\mu 2(\gamma)}}=38.408 \pm 0.007(\mu s)^{-1}
$$

and of the kaon (Antonelli et al. 2010b

$$
\Gamma_{K_{\mu 2(\gamma)}}=51.25 \pm 0.16(\mu s)^{-1},
$$

2 Based on the results from Allton et al. (2008); Aoki et al. (2009); Aubin et al. (2008, 2004); Bazavov et al. (2010 2009); Beane et al. (2007); Bernard et al. (2007); Blossier et al. (2009); Dürr et al. (2010); Follana et al. (2008); Göckeler et al. (2006); Lellouch (2009); Mawhinney (2009); Noaki et al. (2009).
Cirigliano and Neufeld (2011) obtained

$$
\begin{aligned}
\frac{\left|V_{u s}\right| F_{K}}{\left|V_{u d}\right| F_{\pi}} & =0.23922(25) \times\left(\frac{\Gamma_{K_{\ell 2(\gamma)}}}{\Gamma_{\pi_{\ell 2(\gamma)}}}\right)^{1 / 2} \\
& =0.2763 \pm 0.0005 .
\end{aligned}
$$

Finally, taking as reference value for $F_{K} / F_{\pi}$ the FLAG average in Eq. 4.12), the ratio of the CKM matrix elements is given by (Cirigliano and Neufeld, 2011)

$$
\frac{\left|V_{u s}\right|}{\left|V_{u d}\right|}=0.2316 \pm 0.0012 \text {. }
$$

3. The ratio $R_{e / \mu}^{(K, \pi)}$

In a first systematic calculation to $\mathcal{O}\left(e^{2} p^{4}\right)$, the coefficients $c_{2}^{(P)}, c_{3}^{(P)}, c_{4}^{(P)}, \tilde{c}_{2}^{(P)}$ were determined by Cirigliano and Rosell $(2007 \mathrm{a} b)$. This allowed the determination of the ratios $R_{e / \mu}^{(P)}=\Gamma_{P_{e 2(\gamma)}} / \Gamma_{P_{\mu 2(\gamma)}}(P=\pi, K)$ with an unprecedented theoretical accuracy. In the $\mathrm{SM}$, the ratios $R_{e / \mu}^{(P)}$ are helicity suppressed as a consequence of the $V-A$ structure of the charged currents, constituting sensitive probes of New Physics. The two-loop effective theory results were complemented by a large- $N_{C}$ calculation of an associated counterterm and a summation of leading logarithms $\alpha^{n} \ln ^{n}\left(m_{\mu} / m_{e}\right)$ (Marciano and Sirlin, 1993) giving (Cirigliano and Rosell, 2007a|b)

$$
\begin{aligned}
& R_{e / \mu}^{(\pi)}=(1.2352 \pm 0.0001) \times 10^{-4}, \\
& R_{e / \mu}^{(K)}=(2.477 \pm 0.001) \times 10^{-5} .
\end{aligned}
$$

In the case of $R_{e / \mu}^{(K)}$ the uncertainty arising from matching was increased by a factor of four to account for higherorder chiral corrections of $\mathcal{O}\left(e^{2} p^{6}\right)$. The central value of $R_{e / \mu}^{(\pi)}$ is in agreement with the results of previous calculations (Finkemeier, 1996; Marciano and Sirlin, 1993), pushing the theoretical uncertainty below $10^{-4}$. The discrepancy with a previous determination of $R_{e / \mu}^{(K)}$ can be traced back to inconsistencies in the analysis of Finkemeier (1996).

The above theoretical results are compatible with current experimental measurements Ambrosino et al. 2009a; Britton et al., 1994, 1992, Czapek et al., 1993, Lazzeroni et al. |2011) averaging to

$$
\begin{aligned}
& \left.R_{e / \mu}^{(\pi)}\right|_{\exp }=(1.230 \pm 0.004) \times 10^{-4}, \\
& \left.R_{e / \mu}^{(K)}\right|_{\exp }=(2.488 \pm 0.012) \times 10^{-5} .
\end{aligned}
$$

They provide a clean basis to detect or constrain nonstandard physics in these channels by comparison with upcoming measurements, which will push the fractional uncertainty from $0.3 \%$ to $0.05 \%$ in $R_{e / \mu}^{(\pi)}$ (Počanić et al. 2009 Sher et al. 2009 and from $0.5 \%$ to $0.4 \%$ in $R_{e / \mu}^{(K)}$ (Collazuol, 2009). 
B. $K_{\ell 2 \gamma}$

The radiative leptonic decays $K_{\ell 2 \gamma}$,

$$
K^{+}(p) \rightarrow \ell^{+}\left(p_{\ell}\right) \nu_{\ell}\left(p_{\nu}\right) \gamma(q), \quad \ell=e, \mu
$$

allow one to probe the low-energy structure of QCD and its anomalous couplings. The matrix element reads

$$
T=-i e G_{F} V_{u s}^{*} \epsilon_{\mu}^{*}\left(F_{K} L^{\mu}-H^{\mu \nu} l_{\nu}\right)
$$

where $\epsilon^{\mu}$ denotes the photon polarization vector and the other quantities are given by $(W=p-q)$

$$
\begin{aligned}
L^{\mu}= & m_{\ell} \bar{u}\left(p_{\nu}\right)\left(1+\gamma_{5}\right)\left(\frac{2 p^{\mu}}{2 p \cdot q}-\frac{2 p_{\ell}^{\mu}+\not \gamma^{\mu}}{2 p_{\ell} \cdot q}\right) v\left(p_{\ell}\right), \\
l^{\mu}=\bar{u}\left(p_{\nu}\right) \gamma^{\mu}\left(1-\gamma_{5}\right) v\left(p_{\ell}\right), & \\
H^{\mu \nu}=-\frac{1}{\sqrt{2} M_{K}} & {\left[i V\left(W^{2}\right) \epsilon^{\mu \nu \alpha \beta} q_{\alpha} p_{\beta}\right.} \\
& \left.\quad-A\left(W^{2}\right)\left(q \cdot W g^{\mu \nu}-W^{\mu} q^{\nu}\right)\right] .
\end{aligned}
$$

$V\left(W^{2}\right)$ and $A\left(W^{2}\right)$ denote the vector and axial-vector ${ }^{3}$ Lorentz-invariant form factors characterizing the general decomposition of the correlator of weak and electromagnetic currents,

$$
\begin{aligned}
V_{\mu}^{\mathrm{w}} & =\bar{s} \gamma_{\mu} u, \quad A_{\mu}^{\mathrm{w}}=\bar{s} \gamma_{\mu} \gamma_{5} u, \\
V_{\mu}^{\mathrm{em}} & =(2 \bar{u} u-\bar{d} d-\bar{s} s) / 3,
\end{aligned}
$$

between the vacuum and the one-kaon state at $q^{2}=0$, i.e., for real photons (Bijnens et al., 1993):

$$
\begin{aligned}
& \Pi_{\mu \nu}(q, p)= \\
& \int d^{4} x e^{i q x}\left\langle 0\left|T\left(V_{\mu}^{\mathrm{em}}(x)\left(V_{\nu}^{\mathrm{w}}(0)-A_{\nu}^{\mathrm{w}}(0)\right)\right)\right| K^{+}(p)\right\rangle .
\end{aligned}
$$

The explicit relation of $A\left(W^{2}\right)$ and $V\left(W^{2}\right)$ to the above correlator can be found in the appendix of Bijnens et al. (1993). The amplitude (4.21) is the sum of two terms: the so-called "inner-bremsstrahlung" (IB) term (proportional to $L^{\mu}$ ), uniquely determined in terms of the nonradiative amplitude, and the "structure-dependent" (SD) term proportional to $H^{\mu \nu}$. Correspondingly, the spinaveraged differential decay distribution can be decomposed into three terms (IB, SD, and INT, the latter denoting the interference of IB and SD amplitudes):

$$
\begin{aligned}
& \frac{d^{2} \Gamma}{d x d y}=A_{\mathrm{IB}} f_{\mathrm{IB}}(x, y) \\
& +\frac{A_{\mathrm{SD}}}{2}\left[(V+A)^{2} f_{\mathrm{SD}^{+}}(x, y)+(V-A)^{2} f_{\mathrm{SD}^{-}}(x, y)\right] \\
& -\frac{A_{\mathrm{INT}^{-}}}{\sqrt{2}}\left[(V+A) f_{\mathrm{INT}^{+}}(x, y)+(V-A) f_{\mathrm{INT}^{-}}(x, y)\right] .
\end{aligned}
$$

\footnotetext{
${ }^{3}$ Note that we are using here the normalization of $V$ and $A$ adopted in Nakamura et al. (2010).
}

Here

$$
\begin{aligned}
A_{\mathrm{IB}} & =4 \frac{m_{\ell}^{2}}{M_{K}^{2}}\left(\frac{F_{K}}{M_{K}}\right)^{2} A_{\mathrm{SD}}, \\
A_{\mathrm{SD}} & =\frac{G_{F}^{2}\left|V_{u s}\right|^{2} \alpha}{32 \pi^{2}} M_{K}^{5}, \\
A_{\mathrm{INT}} & =4 \frac{m_{\ell}^{2}}{M_{K}^{2}}\left(\frac{F_{K}}{M_{K}}\right) A_{\mathrm{SD}},
\end{aligned}
$$

the independent kinematical variables are

$$
x=\frac{2 p \cdot q}{M_{K}^{2}}, \quad y=\frac{2 p \cdot p_{\ell}}{M_{K}^{2}},
$$

and the functions $f_{\mathrm{IB}}, f_{\mathrm{SD}^{ \pm}}$, and $f_{\mathrm{INT}^{ \pm}}$can be found in Bijnens et al. (1993). Note that the inner-bremsstrahlung and interference terms in the rate 4.25 are proportional to the helicity suppression factor $\left(m_{\ell} / M_{K}\right)^{2}$, so that $K_{e 2 \gamma}$ is dominated by the structure-dependent contribution. On the other hand, $K_{\mu 2 \gamma}$, while sensitive to interference and structure-dependent terms, is dominated by internal bremsstrahlung.

Internal bremsstrahlung is fixed in terms of a single hadronic input, namely the kaon decay constant $F_{K}$. The form factors $V\left(W^{2}\right)$ and $A\left(W^{2}\right)$ can be calculated in CHPT, with $V\left(W^{2}\right)$ arising from the anomalous sector. The first non-trivial contributions arise at $\mathcal{O}\left(p^{4}\right)$ Bijnens et al. 1993):

$$
\begin{aligned}
A_{p^{4}} & =\frac{4 \sqrt{2} M_{K}}{F_{0}}\left(L_{9}^{r}+L_{10}^{r}\right)=0.042, \\
V_{p^{4}} & =\frac{\sqrt{2} M_{K}}{8 \pi^{2} F_{0}}=0.096,
\end{aligned}
$$

where the numerical values were obtained by using the central values $F_{0}=F_{\pi}, L_{9}^{r}\left(\mu=M_{\rho}\right)=6.9 \times 10^{-3}$, and $L_{10}^{r}\left(\mu=M_{\rho}\right)=-5.5 \times 10^{-3}$.

The contributions of $\mathcal{O}\left(p^{6}\right)$ to the vector form factor involve one-loop graphs in the anomalous sector as well as $\mathcal{O}\left(p^{6}\right)$ counterterms. They were worked out first by Ametller et al. (1993) and later confirmed by Geng et al. (2004). To this order, $V\left(W^{2}\right)$ acquires a non-trivial dependence on $W^{2}=M_{K}^{2}(1-x)$, which can be approximated by the linear parametrization

$$
V\left(W^{2}\right)=V_{0}[1+\tilde{\lambda}(1-x)] .
$$

Geng et al. (2004) use estimates of the LECs in the anomalous sector from vector meson dominance and the chiral quark model. Their results can be summarized as:

$$
\begin{aligned}
\left(V_{0}\right)_{p^{4}+p^{6}} & =0.078 \pm 0.005 \\
\tilde{\lambda} & =0.3 \pm 0.1
\end{aligned}
$$

Our estimate of the uncertainty was obtained by looking at the spread in results obtained within different models.

The contributions of $\mathcal{O}\left(p^{6}\right)$ to $A\left(W^{2}\right)$ were worked out first by Geng et al. (2004). They involve two-loop graphs 
with insertion of the lowest-order CHPT Lagrangian $\mathcal{L}_{2}$, one-loop graphs with one insertion of $\mathcal{L}_{4}$, and tree-level graphs from $\mathcal{L}_{6}$. Geng et al. (2004) find that $A\left(W^{2}\right)$ is essentially flat in $W^{2}$ and receives a correction of about $20 \%$ in the normalization, with an uncertainty induced by the LECs at the percent level [the subscript 0 indicates that we quote the $W$-independent part of $\left.A\left(W^{2}\right)\right]$ :

$$
\left(A_{0}\right)_{p^{4}+p^{6}}=0.034
$$

The $\mathcal{O}\left(p^{6}\right)$ corrections are of the expected size for both $V$ and $A$ form factors.

Experimentally, the $K_{e 2 \gamma}$ mode provides the best constraint on the combination $V_{0}+A_{0}$ (Nakamura et al. 2010). The data are dominated by the $1484 \pm 63$ events collected by KLOE (Ambrosino et al. 2009a) in the range $10 \mathrm{MeV}<E_{\gamma}^{\mathrm{cms}}<250 \mathrm{MeV}$. A fit to the measured spectrum leads to the results

$$
\begin{aligned}
V_{0}+A_{0} & =0.125 \pm 0.007_{\text {stat }} \pm 0.001_{\text {syst }} \\
\tilde{\lambda} & =0.38 \pm 0.20_{\text {stat }} \pm 0.02_{\text {syst }}
\end{aligned}
$$

which are in excellent agreement with the $\mathcal{O}\left(p^{6}\right)$ CHPT predictions [from the work of Geng et al. (2004) we extract $\left(V_{0}+A_{0}\right)_{p^{4}+p^{6}}=0.112 \pm 0.005$, where the uncertainty captures the model dependence in the LECs entering the $p^{6}$ calculation]. However, the $K_{\mu 2 \gamma}$ extraction of the combination $V_{0}+A_{0}$ differs from the $K_{e 2 \gamma}$ value (Adler et al., 2000):

$$
V_{0}+A_{0}=0.165 \pm 0.007_{\text {stat }} \pm 0.011_{\text {syst }}
$$

assuming $V+A=V_{0}+A_{0}$, i.e., no dependence on $W^{2}$.

Until very recently, the combination $V_{0}-A_{0}$ was only loosely constrained by $K_{\ell 2 \gamma}$ measurements, the best determination, $V_{0}-A_{0}=0.077 \pm 0.028$ (consistent with CHPT), coming from $K \rightarrow \mu \nu e^{+} e^{-}$(Poblaguev et al. 2002). The situation has changed with the recent results from ISTRA+ reported in Tchikilev et al. (2010) and Duk et al. (2011). They studied the $K_{\mu 2 \gamma}$ mode in a kinematic region in which the interference terms can be extracted, thus providing direct sensitivity to the combination $V_{0}-A_{0}$. Different analyses assuming constant form factors lead to (Tchikilev et al. 2010)

$$
V_{0}-A_{0}=0.126 \pm 0.027_{\text {stat }} \pm 0.047_{\text {syst }},
$$

and (Duk et al. 2011)

$$
V_{0}-A_{0}=0.21 \pm 0.04_{\text {stat }} \pm 0.04_{\text {syst }},
$$

respectively, about 1.5 and 3 sigmas above the CHPT prediction $V_{0}-A_{0}=0.044 \pm 0.010$, where we take as uncertainty the difference between the central values at $\mathcal{O}\left(p^{6}\right)$ and $\mathcal{O}\left(p^{4}\right)$. At this stage it is clearly premature to claim a serious tension between data and theory.

\section{C. $K_{\ell 3}$}

The photon-inclusive decay rate for all four $K \rightarrow \pi \ell \nu$ modes $\left(K=K^{ \pm}, K^{0}, \overline{K^{0}} ; \ell=e, \mu\right)$ can be written as

$$
\begin{aligned}
\Gamma_{K_{\ell 3(\gamma)}=} & \frac{G_{\mathrm{F}}^{2}\left|V_{u s}\right|^{2} M_{K}^{5} C_{K}^{2}}{128 \pi^{3}} S_{\mathrm{EW}}\left|f_{+}^{K^{0} \pi^{-}}(0)\right|^{2} \\
& \times I_{K \ell}^{(0)}\left(\lambda_{i}\right)\left(1+\delta_{\mathrm{EM}}^{K \ell}+\delta_{\mathrm{SU}(2)}^{K \pi}\right)
\end{aligned}
$$

The Clebsch-Gordan coefficient $C_{K}$ differs for neutral and charged kaons $\left(C_{K}=1\right.$ for $K_{\ell 3}^{0}$ and $C_{K}=1 / \sqrt{2}$ for $K_{\ell 3}^{+}$), while $I_{K \ell}^{(0)}$ is a phase space integral depending on slope and curvature of the form factors $f_{ \pm}^{K \pi}(t)$, defined by the QCD matrix elements

$$
\begin{aligned}
&\left\langle\pi\left(p_{\pi}\right)\left|\bar{s} \gamma_{\mu} u\right| K\left(p_{K}\right)\right\rangle= \\
&\left(p_{\pi}+p_{K}\right)_{\mu} f_{+}^{K \pi}(t)+\left(p_{K}-p_{\pi}\right)_{\mu} f_{-}^{K \pi}(t),
\end{aligned}
$$

where $t=\left(p_{K}-p_{\pi}\right)^{2}=\left(p_{\ell}+p_{\nu}\right)^{2}$. As usual, the vector form factor of the $K^{0}$ decay at zero momentum transfer has been pulled out in Eq. (4.37). The strong isospinbreaking correction is defined as

$$
\delta_{\mathrm{SU}(2)}^{K \pi}=\left(f_{+}^{K \pi}(0) / f_{+}^{K^{0} \pi^{-}}(0)\right)^{2}-1 .
$$

The long-distance electromagnetic corrections

$$
\delta_{\mathrm{EM}}^{K \ell}=\delta_{\mathrm{EM}}^{K \ell}\left(\mathcal{D}_{3}\right)+\delta_{\mathrm{EM}}^{K \ell}\left(\mathcal{D}_{4-3}\right)
$$

receive contributions from three-particle and fourparticle final states. In the following sections we review the theoretical quantities appearing in the expression of the decay rate and then combine this information with experimental input to extract the CKM element $V_{u s}$.

\section{Electromagnetic effects in $K_{\ell 3(\gamma)}$ decays}

The calculation of the electromagnetic contributions to $\mathcal{O}\left(e^{2} p^{2}\right)$ in $K_{\ell 3}$ decays using the methods of CHPT with virtual photons and leptons (Knecht et al. , 2000) was presented in Cirigliano et al. (2002). Based on this analysis, full numerical results on the $K_{e 3}$ decay modes were given in Cirigliano et al. (2004b), adopting a specific prescription for treating real photon emission and a specific factorization scheme for soft photons. This approach resulted in the partial inclusion of higher-order terms in the chiral expansion. In a recent publication (Cirigliano et al. 2008b), the numerical analysis of electromagnetic corrections was extended to a complete study of $K_{\mu 3}$ decays. At the same time, previous results for the $K_{e 3}$ modes were updated using the new estimates of electromagnetic LECs (Ananthanarayan and Moussallam, 2004 Descotes-Genon and Moussallam, 2005) that affect the structure-dependent electromagnetic contributions. Rather than using the soft-photon factorization procedure of Cirigliano et al. (2002), the analysis was performed at fixed chiral order $e^{2} p^{2}$. 
TABLE II Electromagnetic corrections to $K_{\ell 3}$ decay rates (Cirigliano et al. 2008b). $\mathcal{D}_{3}$ refers to three-particle phase space, $\mathcal{D}_{4}$ to four-body kinematics.

\begin{tabular}{|l|c|c|c|}
\hline & $\delta_{\mathrm{EM}}^{K \ell}\left(\mathcal{D}_{3}\right)(\%)$ & $\delta_{\mathrm{EM}}^{K \ell}\left(\mathcal{D}_{4-3}\right)(\%)$ & $\delta_{\mathrm{EM}}^{K \ell}(\%)$ \\
\hline$K_{e 3}^{0}$ & 0.50 & 0.49 & $0.99 \pm 0.22$ \\
$K_{e 3}^{ \pm}$ & -0.35 & 0.45 & $0.10 \pm 0.25$ \\
$K_{\mu 3}^{0}$ & 1.38 & 0.02 & $1.40 \pm 0.22$ \\
$K_{\mu 3}^{ \pm}$ & 0.007 & 0.009 & $0.016 \pm 0.25$ \\
\hline
\end{tabular}

Table II summarizes the numerical results for the long-distance radiative corrections. Two characteristic features can be understood by qualitative arguments. Firstly, the electromagnetic corrections for the neutral $K$ decays are expected to be positive and sizable on account of the final-state Coulomb interaction between $\ell^{+}$and $\pi^{-}$producing a correction factor of $\pi \alpha / v_{\ell^{+} \pi^{-}}^{\text {rel }} \sim 2 \%$ over most of the Dalitz plot. While the exact correction and the relative size of $K_{\mu 3}^{0}$ and $K_{e 3}^{0}$ depend on other effects such as the emission of real photons, the qualitative expectation based on the Coulomb interaction is confirmed by the detailed calculation. Secondly, the large hierarchy $\delta_{\mathrm{EM}}^{K \mu}\left(\mathcal{D}_{4-3}\right) \ll \delta_{\mathrm{EM}}^{K e}\left(\mathcal{D}_{4-3}\right)$ admits a simple interpretation in terms of bremsstrahlung off the charged lepton in the final state. The probability of emitting soft photons is a function of the lepton velocity $v_{\ell}$ which becomes logarithmically singular as $v_{\ell} \rightarrow 1$, thus enhancing the electron emission. For typical values of $v_{\ell}$ in $\mathcal{D}_{4-3}$, the semiclassical emission probability implies $\delta_{\mathrm{EM}}^{K e}\left(\mathcal{D}_{4-3}\right) / \delta_{\mathrm{EM}}^{K \mu}\left(\mathcal{D}_{4-3}\right) \sim 20 \rightarrow 40$.

The theoretical uncertainties assigned to the $\delta_{\mathrm{EM}}^{K \ell}$ in Table II arise from two sources: the input parameters (LECs and form factor parameters) used in the calculation and unknown higher-order terms in the chiral expansion (the latter would require a complete analysis at order $\left.e^{2} p^{4}\right)$. For a detailed discussion of the error estimate, we refer to Cirigliano et al. (2008b).

The differential decay distribution can be written in the form

$$
\begin{aligned}
\frac{d^{2} \Gamma}{d y d z}= & \frac{G_{\mathrm{F}}^{2}\left|V_{u s}\right|^{2} M_{K}^{5} C_{K}^{2}}{128 \pi^{3}} S_{\mathrm{EW}}\left|f_{+}^{K \pi}(0)\right|^{2} \\
& \times\left[\bar{\rho}^{(0)}(y, z)+\delta \bar{\rho}^{\mathrm{EM}}(y, z)\right],
\end{aligned}
$$

where the Lorentz invariants $y=2 p_{K} \cdot p_{\ell} / M_{K}^{2}=2 E_{\ell} / M_{K}$ and $z=2 p_{\pi} \cdot p_{K} / M_{K}^{2}=2 E_{\pi} / M_{K}$ are related to the energy of the charged lepton and of the pion, respectively, measured in the rest frame of the kaon. Here $\bar{\rho}^{(0)}(y, z)$ represents the Dalitz plot density in absence of electromagnetic corrections, while $\delta \bar{\rho}^{\mathrm{EM}}(y, z)$ accounts for electromagnetic effects. Illustrative figures displaying the relative size of the electromagnetic corrections over the Dalitz plot can be found in Cirigliano et al. (2008b). It turns out that the corrections to the Dalitz distributions can be locally quite large $(\sim 10 \%)$ and do not have a definite sign, implying cancellations in the integrated total electromagnetic corrections.

\section{Quark mass ratios and $K_{\ell 3}$ decays}

The isospin-breaking correction to $K_{\ell 3}$ decays takes the form (Cirigliano et al., 2002)

$$
\delta_{\mathrm{SU}(2)}^{K^{ \pm} \pi^{0}}=2 \sqrt{3}\left(\varepsilon^{(2)}+\varepsilon_{\mathrm{S}}^{(4)}+\varepsilon_{\mathrm{EM}}^{(4)}+\ldots\right) .
$$

It is dominated by the lowest-order $\pi^{0}-\eta$ mixing angle $\varepsilon^{(2)}$ defined in Eq. 4.11). The NLO corrections $\varepsilon_{S}^{(4)}$ (or$\operatorname{der} p^{4}$ ) and $\varepsilon_{\mathrm{FM}}^{(4)}$ (order $e^{2} p^{2}$ ) were computed in Gasser and Leutwyler (1985b) and Neufeld and Rupertsberger (1995), respectively. The explicit expressions for these quantities can be found in Cirigliano et al. (2002). The dots refer to NNLO contributions (arising at order $p^{6}$ ), for which the latest results can be found in Bijnens and Ghorbani (2007b). Working strictly to $\mathcal{O}\left(p^{4}\right)$ in CHPT and neglecting the tiny contribution $\varepsilon_{\mathrm{EM}}^{(4)}$, one can relate $\delta_{\mathrm{SU}(2)}^{K^{ \pm} \pi^{0}}$ to quark mass ratios by

$$
\delta_{\mathrm{SU}(2)}^{K^{ \pm} \pi^{0}}=\frac{3}{2} \frac{1}{Q^{2}}\left[\frac{M_{K}^{2}}{M_{\pi}^{2}}+\frac{\chi_{p^{4}}}{2}\left(1+\frac{m_{s}}{\hat{m}}\right)\right],
$$

where $Q^{2}=\left(m_{s}^{2}-\widehat{m}^{2}\right) /\left(m_{d}^{2}-m_{u}^{2}\right)=R\left(m_{s} / \widehat{m}+1\right) / 2$, $M_{K, \pi}$ are the isospin-limit meson masses and $\chi_{p^{4}}=0.219$ is a calculable loop correction. Thus, $\delta_{\mathrm{SU}(2)}^{K^{ \pm} \pi^{0}}$ is essentially determined by $Q^{2}$ (the uncertainty in $m_{s} / \hat{m}$ affects $\delta_{\mathrm{SU}(2)}^{K^{ \pm} \pi^{0}}$ negligibly due to the smallness of $\left.\chi_{p^{4}}\right)$.

The standard strategy up to now has been to use all known information on light quark masses to predict $\delta_{\mathrm{SU}(2)}^{K^{ \pm} \pi^{0}}$ using Eq. 44.43. The double ratio $Q^{2}$ can be expressed in terms of meson masses and a purely electromagnetic contribution (Gasser and Leutwyler, 1985a),

$$
Q^{2}=\frac{\Delta_{K \pi} M_{K}^{2}\left(1+\mathcal{O}\left(m_{q}^{2}\right)\right)}{M_{\pi}^{2}\left[\Delta_{K^{0} K^{+}}+\Delta_{\pi^{+} \pi^{0}}-\left(\Delta_{K^{0} K^{+}}+\Delta_{\pi^{+} \pi^{0}}\right) \mathrm{EM}\right]},
$$

where $\Delta_{P Q}=M_{P}^{2}-M_{Q}^{2}$. The electromagnetic term $\left(\Delta_{K^{0} K^{+}}+\Delta_{\pi^{+} \pi^{0}}\right)_{\mathrm{EM}}$ vanishes to lowest order $e^{2} p^{0}$ (Dashen, 1969). A calculation at $\mathcal{O}\left(e^{2} p^{2}\right)$ gives (Neufeld and Rupertsberger, 1995, Urech, 1995)

$$
\begin{aligned}
& \left(\Delta_{K^{0} K^{+}}+\Delta_{\pi^{+} \pi^{0}}\right)_{\mathrm{EM}}=e^{2} M_{K}^{2}\left[16 Z L_{5}^{r}(\mu)\right. \\
& +\frac{4}{3}\left(K_{5}+K_{6}\right)^{r}(\mu)-8\left(K_{10}+K_{11}\right)^{r}(\mu) \\
& \left.+\frac{1}{4 \pi^{2}}\left(3 \ln \frac{M_{K}^{2}}{\mu^{2}}-4+2 Z \ln \frac{M_{K}^{2}}{\mu^{2}}\right)\right]+\mathcal{O}\left(e^{2} M_{\pi}^{2}\right)
\end{aligned}
$$

The current estimates (Ananthanarayan and Moussallam, 2004) of the electromagnetic LECs appearing in 
this expression imply a large deviation from Dashen's limit, $\left(\Delta_{K^{0} K^{+}}+\Delta_{\pi^{+} \pi^{0}}\right)_{\mathrm{EM}}=-1.5 \Delta_{\pi^{+} \pi^{0}}$, which implies $Q=20.7 \pm 1.2$ (Kastner and Neufeld, 2008). Such a small value of $Q$ [compared to $Q=22.7 \pm 0.8$ given by Leutwyler (1996)] was also supported by other studies $[Q=22.0 \pm 0.6$ in Bijnens and Prades (1997) and $Q \simeq 20$ in Amorós et al. (2001)]. It should be noted, however, that the rather large value $Q=23.2$ was obtained from an analysis of $\eta \rightarrow 3 \pi$ at two loops (Bijnens and Ghorbani, 2007a). On the other hand, the non-lattice determinations of the second input parameter $m_{s} / \widehat{m} \sim 24$ have remained rather stable over the last years. Combining $Q=20.7 \pm 1.2$ with $m_{s} / \widehat{m}=24.7 \pm 1.1$, Kastner and Neufeld (2008) found $R=33.5 \pm 4.3$ and finally $\delta_{\mathrm{SU}(2)}^{K^{ \pm} \pi^{0}}=0.058 \pm 0.008$.

It is worth stressing that the present precision of the decay rates and of the radiative corrections permits also an "experimental" determination of $\delta_{\mathrm{SU}(2)}^{K^{ \pm}} \pi^{0}$, which can be used as a constraint on the quark mass ratio $Q$ via the formula 4.43). Combining recent $K_{\ell 3}$ data (Antonelli et al. 2010b) with the expression

$\delta_{\mathrm{SU}(2)}^{K^{ \pm} \pi^{0}}=\frac{2 \Gamma_{K_{\ell 3}^{+}}}{\Gamma_{K_{\ell 3}^{0}}} \frac{I_{K^{0} \ell}}{I_{K+\ell}}\left(\frac{M_{K^{0}}}{M_{K^{+}}}\right)^{5}-1-\left(\delta_{\mathrm{EM}}^{K^{+} \ell}-\delta_{\mathrm{EM}}^{K^{0} \ell}\right)$

one obtains $\delta_{\mathrm{SU}(2) \exp }^{K^{ \pm} \pi^{0}}=0.054 \pm 0.008$, in perfect agreement with the value obtained from quark mass ratios.

Alternatively, one may use the $N_{f}=2+1$ lattice average (Colangelo et al. , 2011) $m_{s} / \widehat{m}=27.4 \pm 0.4$ being considerably larger than the values obtained with nonlattice methods. Combined with $Q=22.8 \pm 1.2$ from the same data compilation, Eq. 4.43 yields $\delta_{\mathrm{SU}(2)}^{K^{ \pm} \pi^{0}}=$ $0.048 \pm 0.006$, still consistent with the experimentally determined result.

\section{Form factors and phase space integrals}

Calculation of the phase space integrals $I_{K \ell}$ requires knowing the momentum dependence of the form factors. The vector form factor $f_{+}^{K \pi}(t)$ defined in Eq. 4.38 represents the p-wave projection of the crossed-channel matrix element $\left\langle 0\left|\bar{s} \gamma^{\mu} u\right| K \pi\right\rangle$ whereas the s-wave projection is described by the scalar form factor

$$
f_{0}(t)=f_{+}(t)+\frac{t}{M_{K}^{2}-M_{\pi}^{2}} f_{-}(t) .
$$

It is convenient to normalize all the form factors to $f_{+}^{K^{0} \pi^{-}}(0)$ (denoted $f_{+}(0)$ in the following). In terms of the normalized form factors $\bar{f}_{i}(t) \equiv f_{i}(t) / f_{+}(0)$, the phase space integrals read

$$
\begin{aligned}
I_{K \ell}= & \frac{2}{3} \int_{m_{\ell}^{2}}^{t_{0}} \frac{d t}{M_{K}^{8}} \bar{\lambda}^{3 / 2}\left(1+\frac{m_{\ell}^{2}}{2 t}\right)\left(1-\frac{m_{\ell}^{2}}{2 t}\right)^{2} \\
& \times\left(\bar{f}_{+}^{2}(t)+\frac{3 m_{\ell}^{2} \Delta_{K \pi}^{2}}{\left(2 t+m_{\ell}^{2}\right) \bar{\lambda}} \bar{f}_{0}^{2}(t)\right)
\end{aligned}
$$

with $\bar{\lambda}=\left(t-\left(M_{K}+M_{\pi}\right)^{2}\right)\left(t-\left(M_{K}-M_{\pi}\right)^{2}\right)$.

Traditionally, a polynomial parametrization has been used for the form factors,

$$
\bar{f}_{+, 0}(t)=1+\lambda_{+, 0}^{\prime} \frac{t}{M_{\pi^{+}}^{2}}+\frac{1}{2} \lambda_{+, 0}^{\prime \prime}\left(\frac{t}{M_{\pi^{+}}^{2}}\right)^{2}+\ldots,
$$

where $\lambda_{+, 0}^{\prime}$ and $\lambda_{+, 0}^{\prime \prime}$ are the slope and curvature, respectively. Fits to the experimental distributions of $K_{\ell 3}$ decays allow to extract the parameters $\lambda_{+}^{\prime}, \lambda_{+}^{\prime \prime}$, and $\lambda_{0}^{\prime}$. The resulting uncertainty on the phase space integrals is at the level of $0.12 \%$ for $I_{K e}$ and $0.30 \%$ for $I_{K \mu}$ (Antonelli et al. 2010b). This affects the extraction of $V_{u s}$ at the level of $0.06 \%\left(K_{e 3}\right)$ and $0.15 \%\left(K_{\mu 3}\right)$.

Other form factor parametrizations have been proposed, in which, by using physical inputs, specific relations between the slope, the curvature and all the higherorder terms of the Taylor expansion 4.49 are imposed. This allows to reduce the correlations between the fitted slope parameters: only one parameter is fitted for each form factor. Explicit examples used to analyze data include the pole parametrization, dispersive parametrizations (Abouzaid et al. 2010; Bernard et al., 2006, 2009), and the so-called $z$-parametrization (Hill, 2006).

\section{The $K_{\ell 3}$ scalar form factor}

SM predictions for the slope parameter $\lambda_{0}^{\prime}$ of the scalar form factor of $K_{\ell 3}$ decays were obtained by using different approaches. In the isospin limit, the combination of a two-loop result in chiral perturbation theory (Bijnens and Talavera, 2003) with an updated estimate of the relevant $p^{6}$ low-energy couplings based on Cirigliano et al. (2005) and Cirigliano et al. (2003b) gave the result $\lambda_{0}^{\prime}=\left(13.9_{-0.4}^{+1.3} \pm 0.4\right) \times 10^{-3}$ (Kastner and Neufeld, 2008).

Dispersive methods were employed by several authors. ${ }^{4}$ Typical numbers for the resulting scalar slope parameter are: $\lambda_{0}^{\prime}=(14.7 \pm 0.4) \times 10^{-3}$, (Jamin et al. 2006), and $\lambda_{0}^{\prime}=13.71 \times 10^{-3}$ (Bernard et al. 2011).

The low-energy theorem of Callan and Treiman (Callan and Treiman, 1966, Dashen and Weinstein, 1969) predicts the size of the scalar $K_{\ell 3}$ form factor at the (unphysical) momentum transfer $t=\Delta_{K \pi}$,

$$
f_{0}\left(\Delta_{K \pi}\right)=F_{K} / F_{\pi}+\Delta_{\mathrm{CT}}
$$

with a correction term of $\mathcal{O}\left(m_{u}, m_{d}, e^{2}\right)$. In the isospin limit $\left(m_{u}=m_{d}, e=0\right)$, and at first non-leading order, the tiny value $\Delta_{\mathrm{CT}}=-3.5 \times 10^{-3}$ was computed by Gasser and Leutwyler (1985b). A discussion of higherorder effects on this quantity can be found in Bijnens and

\footnotetext{
${ }^{4}$ See for instance Jamin et al. (2002), Jamin et al. (2004), Jamin et al. (2006), Bernard et al. (2006), Bernard and Passemar (2008), Bernard et al. (2009), Bernard et al. (2011), Abbas et al. (2010).
} 
TABLE III Experimental results for the slope parameter of the scalar $K_{\ell 3}$ form factor, in units of $10^{-3}$.

\begin{tabular}{|c|c|c|}
\hline Experiment & $\lambda_{0}^{\prime}$ & Ref. \\
\hline $\operatorname{ISTRA}+\left(K_{\mu 3}^{-}\right)$ & $17.1 \pm 2.2$ & Yushchenko et al. (2004) \\
\hline $\mathrm{KTeV}\left(K_{L \mu 3}\right)$ & $12.8 \pm 1.8$ & Alexopoulos et al. (2004) \\
\hline $\mathrm{KTeV}\left(K_{L \mu 3}+K_{L e 3}\right)$ & $13.7 \pm 1.3$ & Alexopoulos et al. (2004) \\
\hline NA48 $\left(K_{L \mu 3}\right)$ & $9.5 \pm 1.4$ & Lai et al. (2007) \\
\hline $\operatorname{KLOE}\left(K_{L \mu 3}\right)$ & $9.1 \pm 6.5$ & Ambrosino et al. (2007) \\
\hline $\operatorname{KLOE}\left(K_{L \mu 3}+K_{L e 3}\right)$ & $15.4 \pm 2.2$ & Ambrosino et al. (2007) \\
\hline
\end{tabular}

Talavera (2003) and Kastner and Neufeld (2008). Note that the constraint at the Callan-Treiman point plays an essential role in the dispersive analysis of the scalar form factor (Bernard et al., 2006: Bernard and Passemar, 2008, Jamin et al. 2004, 2006).

The effect of isospin violation and electromagnetic corrections has also been considered (Kastner and Neufeld, 2008). This introduces an additional uncertainty for the values of the slope parameters of at most $\pm 10^{-3}$, mainly due to not yet fully determined low-energy couplings. Combining the loop results given by Bijnens and Ghorbani (2007b) with an estimate of the relevant lowenergy couplings, the difference of the slope parameters of the neutral and charged kaons was found (Kastner and Neufeld, 2008) to be confined to the rather small range $0 \lesssim \lambda_{0}^{\prime}\left(K_{\ell 3}^{\mathrm{v}}\right)-\lambda_{0}^{\prime}\left(K_{\ell 3}^{ \pm}\right) \lesssim 10^{-3}$.

The present experimental situation is displayed in Table III Note that the numbers ${ }^{5}$ shown here are those where the quadratic parametrization $\sqrt{4.49}$ has been used for the simultaneous determination of the vector form factor. The $\mathrm{KTeV}$ data were also reanalyzed by Abouzaid et al. (2010) using the dispersive parametrization. The results $\lambda_{0}^{\prime}=(13.22 \pm 1.39) \times 10^{-3}\left(K_{L \mu 3}\right.$ only $)$ and $\lambda_{0}^{\prime}=(12.95 \pm 1.17) \times 10^{-3}\left(K_{L \mu 3}+K_{L e 3}\right)$ are rather close to the corresponding numbers given in Table III] The analogous procedure for the KLOE data Ambrosino et al. 2007) gave $\lambda_{0}^{\prime}=(14.0 \pm 2.1) \times 10^{-3}\left(K_{L \mu 3}+K_{L e 3}\right)$.

The experimental results for the scalar slope parameter found by ISTRA+, KTeV and KLOE are in agreement with the predictions of the SM. On the other hand, the value found by NA48 can hardly be reconciled with the theoretical numbers. Furthermore, an isospin violation of the size as it would be suggested by the simultaneous validity of the ISTRA+ and NA48 results cannot be explained within the SM (Kastner and Neufeld, 2008). A clarification of the origin of these puzzling results in $K_{\mu 3}$ decays would be highly welcome (Leutwyler, 2009).

\footnotetext{
${ }^{5}$ The ISTRA+ result has been rescaled by $M_{\pi^{+}}^{2} / M_{\pi^{0}}^{2}$.
}

5. $\mathrm{SU}(3)$ breaking effects in $f_{+}^{K^{0} \pi^{-}}(0)$

The value of the $K_{\ell 3}^{0}$ form factor at zero momentum transfer, $f_{+}^{K^{0} \pi^{-}}(0) \equiv f_{+}(0)$, is the missing theoretical ingredient for the extraction of $V_{u s}$. Within CHPT we can break up the form factor according to its expansion in quark masses:

$$
f_{+}(0)=1+f_{p^{4}}+f_{p^{6}}+\ldots .
$$

Deviations from unity (the octet symmetry limit) are of second order in SU(3) breaking Ademollo and Gatto, 1964 Behrends and Sirlin, 1960). The first correction arises at $\mathcal{O}\left(p^{4}\right)$ in CHPT: a finite one-loop contribution (Gasser and Leutwyler, 1985b, Leutwyler and Roos, 1984 ) determines $f_{p^{4}}=-0.0227$ in terms of $F_{\pi}, M_{K}$ and $M_{\pi}$, with essentially no uncertainty. The $p^{6}$ term was first estimated by Leutwyler and Roos (1984) in the quark model framework, leading to

$$
f_{+}(0)_{\mathrm{LR}}=0.961 \pm 0.008 .
$$

Within CHPT, $f_{p^{6}}$ receives contributions from twoloop diagrams, one-loop diagrams with insertion of one vertex from the $p^{4}$ effective Lagrangian, and tree-level diagrams with two insertions from $\mathcal{L}_{p^{4}}$ or one from $\mathcal{L}_{p^{6}}$ (Bijnens and Talavera, 2003, Post and Schilcher, 2002):

$$
f_{p^{6}}=f_{p^{6}}^{2-\mathrm{loop}}(\mu)+f_{p^{6}}^{L_{i} \times \text { loop }}(\mu)+f_{p^{6}}^{\text {tree }}(\mu) .
$$

Individual components depend on the chiral renormalization scale $\mu$, their sum being scale independent. Using $\mu=M_{\rho}=0.77 \mathrm{GeV}$, one has (Bijnens and Talavera, 2003) $f_{p^{6}}^{2-\text { loop }}\left(M_{\rho}\right)+f_{p^{6}}^{L_{i} \times \text { loop }}\left(M_{\rho}\right)=+0.0093 \pm 0.0005$. The $p^{6}$ constants appearing in $f_{p^{6}}^{\text {tree }}$ could be determined phenomenologically (Bijnens and Talavera, 2003), provided the experimental errors on the slope and curvature of the scalar form factor reach the level $\Delta \lambda_{0}^{\prime} \sim 0.001$ and $\Delta \lambda_{0}^{\prime \prime} \sim 0.0001$, which is unfortunately not achievable in the near future. Therefore, further theoretical input on $f_{+}(0)$ is needed.

In Cirigliano et al. (2005) a (truncated) large- $N_{C}$ estimate of $f_{p^{6}}^{\text {tree }}$ was performed. It was based on matching a meromorphic approximation to the $\langle S P P\rangle$ Green function (with poles corresponding to the lowest-lying scalar and pseudoscalar resonances) onto QCD by imposing the correct large-momentum fall-off, both off-shell and on one- and two-pion mass shells. The uncertainty was estimated by varying the matching scale in the range $\mu \in\left[M_{\eta}, 1 \mathrm{GeV}\right]$, leading to

$$
f_{+}(0)_{\mathrm{CHPT}+1 / \mathrm{N}_{\mathrm{C}}}=0.984 \pm 0.012 .
$$

Finally, starting with the work of Bećirević et al. (2005) it has been realized that lattice QCD is a powerful tool to estimate $f_{+}(0)$ at a level of accuracy interesting for phenomenological purposes. Unquenched results are now available with both $N_{f}=2+1$ and $N_{f}=2($ Boyle et al. 2008, 2010; Brommel et al., 2007, Dawson et al., 2006; 


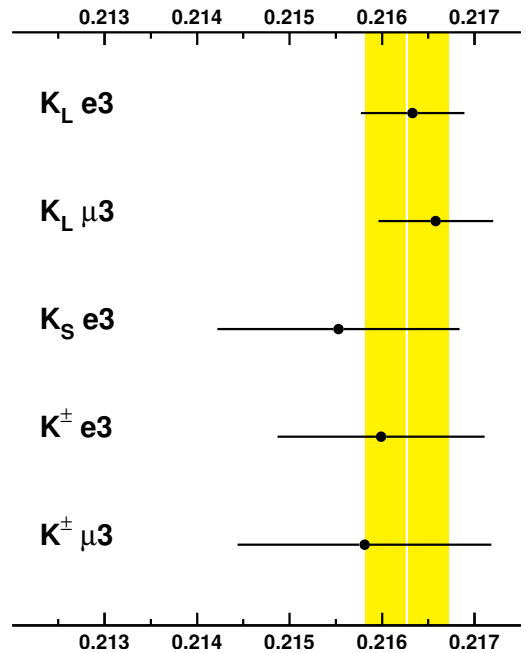

FIG. 2 Compilation of values for $\left|V_{u s}\right| f_{+}(0)$ extracted from all $K_{\ell 3}$ channels. The vertical band denotes the average. From Antonelli et al. (2010a).

Lubicz et al. 2009; Tsutsui et al., 2006). The lattice results agree quite well with the Leutwyler-Roos estimate, while the analytic approaches tend to be higher as a consequence of including the large and positive $(\sim 0.01)$ two-loop effects (Bijnens and Talavera, 2003). In coming years lattice calculations will be performed closer and closer to the physical light quark masses and improved chiral extrapolations can be expected. For our phenomenological extraction of $V_{u s}$ we take as reference value the most recent lattice result with $N_{f}=2+1 \mathrm{dy}$ namical flavors (Boyle et al. 2010), which is also the most precise result for $f_{+}(0)$,

$$
f_{+}(0)_{\mathrm{RBC} / \mathrm{UKQCD}}=0.9599(34)\left({ }_{-47}^{+31}\right)(14),
$$

where the first error is statistical, the second is due to the uncertainties in the chiral extrapolation and the third is an estimate of discretization effects. Currently the dominant systematic uncertainty arises from the extrapolation of lattice results, obtained with unphysical quark masses, to physical light quark masses (Bernard and Passemar, 2010). More progress on $f_{+}(0)$ is expected soon from other lattice collaborations.

\section{Determination of $V_{u s}$ and CKM unitarity tests}

The combination $\left|V_{u s}\right| f_{+}(0)$ can be extracted from both charged and neutral $K$ decays and its value is dominated by $K^{0}$ modes (see Fig. 2). Using the experimental averages from Antonelli et al. (2010a), one obtains

$$
\left|V_{u s}\right| f_{+}(0)=0.2163 \pm 0.0005 \text {. }
$$

Taking as a reference value for $f_{+}(0)$ the lattice result of Boyle et al. (2010), $\left|V_{u s}\right|$ is given by

$$
\left|V_{u s}\right|\left(K_{\ell 3}\right)=0.2255 \pm 0.0005_{\exp } \pm 0.0012_{\text {th }},
$$

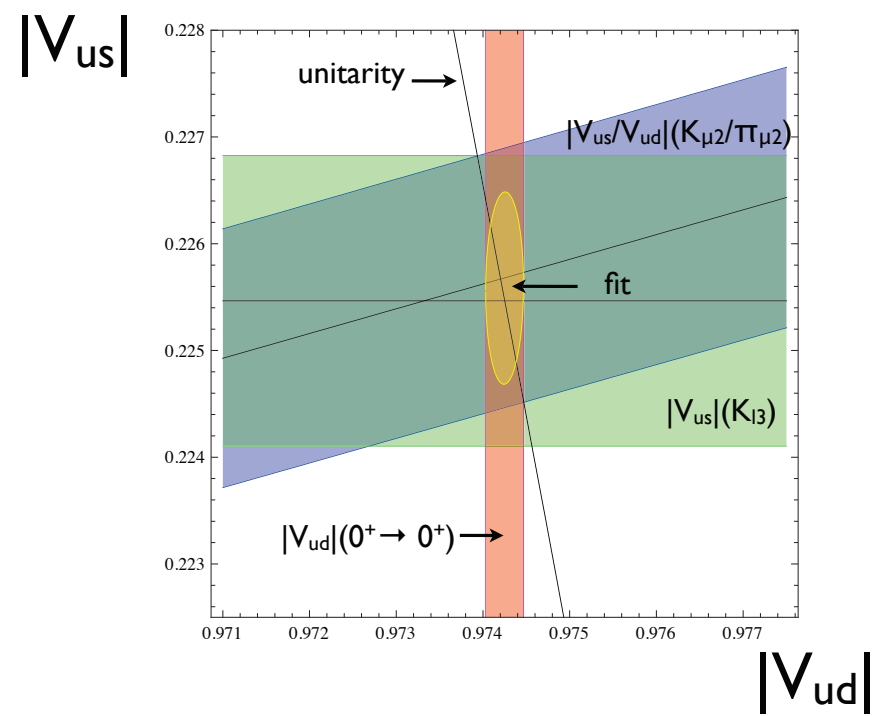

FIG. 3 Graphical representation of the current status of $\left|V_{u d}\right|,\left|V_{u s}\right|$ and the corresponding CKM unitarity test. The horizontal band represents the constraint from $K_{\ell 3}$ decays, the thin vertical band the constraint from $0^{+} \rightarrow 0^{+}$nuclear decays, the oblique band the constraint from $K_{\mu 2} / \pi_{\mu 2}$, and the ellipse is the $1 \sigma$ fit region. From Cirigliano and Neufeld (2011).

where we have explicitly displayed the current experimental and theoretical uncertainties [dominated by $\left.f_{+}(0)\right]$. A smaller value would be obtained with the analytical $f_{+}(0)$ value in Eq. (4.54). As discussed by Antonelli et al. (2010a), one can perform a fit to $\left|V_{u d}\right|$ and $\left|V_{u s}\right|$ using as input the values of $\left|V_{u s}\right|$ from $K_{\ell 3}$ decays [Eq. [4.57]], $\left|V_{u s} / V_{u d}\right|$ from $K_{\ell 2} / \pi_{\ell 2}$ [Eq. (4.17)], and $\left|V_{u d}\right|=0.97425 \pm 0.00022$ from superallowed nuclear $\beta$ transitions (Hardy and Towner, 2009). The outcome is (Cirigliano and Neufeld, 2011)

$$
\begin{aligned}
\left|V_{u d}\right| & =0.97425 \pm 0.00022 \\
\left|V_{u s}\right| & =0.2256 \pm 0.0009
\end{aligned}
$$

with $\chi^{2} /$ ndf $=0.012$ and negligible correlations between $\left|V_{u d}\right|$ and $\left|V_{u s}\right|$. Fig. 3 provides a graphical representation of the various constraints in the $\left|V_{u s}\right|-\left|V_{u d}\right|$ plane and the $1 \sigma$ fit region. These values [together with the negligible contribution from $\left|V_{u b}\right|=0.00393(36)$ (Antonelli et al. [2010b]] can be used to perform a very stringent test of CKM unitarity or, equivalently, of the universality of quark and lepton weak charged-current couplings. For the first-row unitarity sum we find

$$
\Delta_{\mathrm{CKM}}=\left|V_{u d}\right|^{2}+\left|V_{u s}\right|^{2}+\left|V_{u b}\right|^{2}-1=0.0001(6) .
$$

This constraint allows one to set bounds on the effective scale of operators that parametrize new-physics contributions to $\Delta_{\mathrm{CKM}}$ (Cirigliano et al., 2010). The effective scale is constrained to be $\Lambda>11 \mathrm{TeV}$ (90\% C.L.), which puts this low-energy constraint at the same level as the bounds from $Z$-pole measurements. 


\section{T violation in $K_{\mu 3}$ decays}

The transverse muon polarization in $K_{\mu 3}$ decays

$$
P_{T}=\frac{\vec{s}_{\mu} \cdot\left(\vec{p}_{\pi} \times \vec{p}_{\mu}\right)}{\left|\vec{p}_{\pi} \times \vec{p}_{\mu}\right|}
$$

violates $\mathrm{T}$ in the absence of final-state interactions (FSI) (D'Ambrosio and Isidori, 1998). In the case of $K_{L} \rightarrow$ $\pi^{-} \mu^{+} \nu$, with two charged particles in the final state, the electromagnetic interaction generates $\left\langle P_{T}\right\rangle_{\mathrm{FSI}} \sim 10^{-3}$ (Okun and Khriplovich, 1968). In $K_{\mu 3}^{ \pm}$decays, this effect does not exceed $10^{-5}$ (Efrosinin et al., 2000, Zhitnitsky, 1980 and T-violating effects could be important.

The SM CP-violating contribution to $P_{T}$ is very small $\sim 10^{-7}$ (Bigi and Sanda, 2000, Cheng, 1983). Therefore, the measurement of the transverse polarization of muons in $K_{\mu 3}^{ \pm}$is regarded as a sensitive probe for physics beyond the SM (Kohl, 2010, Paton et al., 2006).

The present experimental value (Abe et al., 2006)

$$
P_{T}=-0.0017 \pm 0.0023_{\text {stat }} \pm 0.0011_{\text {syst }}
$$

is consistent with no $\mathrm{T}$ violation and corresponds to the limit $\left|P_{T}\right|<0.0050$ (90\%C.L.). The sensitivity of TREK (Kohl, 2010, Paton et al., 2006) will be able to improve this current upper limit by at least a factor 20 .

D. $K_{\ell 3 \gamma}$

The radiative $K_{\ell 3 \gamma}$ decays $(\ell=e, \mu)$

$$
\begin{aligned}
K^{+}(p) & \rightarrow \pi^{0}\left(p^{\prime}\right) \ell^{+}\left(p_{\ell}\right) \nu_{\ell}\left(p_{\nu}\right) \gamma(q), \\
K^{0}(p) & \rightarrow \pi^{-}\left(p^{\prime}\right) \ell^{+}\left(p_{\ell}\right) \nu_{\ell}\left(p_{\nu}\right) \gamma(q)
\end{aligned}
$$

allow us to perform quantitative tests of CHPT, thanks to theoretical developments over the past couple of decades as well as recent and ongoing high-statistics experimental studies. The decay amplitude can be written as [we focus for definiteness on $K_{e 3 \gamma}^{0}$, the generalization is straightforward and can be found in Bijnens et al. (1993)]:

$$
\begin{aligned}
& T\left(K_{e 3 \gamma}^{0}\right)=\frac{G_{F}}{\sqrt{2}} e V_{u s}^{*} \epsilon_{\mu}(q)^{*} \\
& \quad \times\left[\left(V_{\mu \nu}-A_{\mu \nu}\right) \bar{u}\left(p_{\nu}\right) \gamma^{\nu}\left(1-\gamma_{5}\right) v\left(p_{e}\right)\right. \\
& \left.\quad+\frac{F_{\nu}}{2 p_{e} \cdot q} \bar{u}\left(p_{\nu}\right) \gamma^{\nu}\left(1-\gamma_{5}\right)\left(m_{e}-\not p_{e}-\not q\right) \gamma_{\mu} v\left(p_{e}\right)\right],
\end{aligned}
$$

where the first and second terms correspond to diagrams a) and b), respectively, in Fig. 4. The hadronic matrix elements are defined by $(J=V, A)$

$$
\begin{aligned}
J_{\mu \nu} & =i \int d x e^{i q x}\left\langle\pi^{-}\left(p^{\prime}\right)\left|T\left(V_{\mu}^{\mathrm{em}}(x) J_{\nu}^{\mathrm{w}}(0)\right)\right| K^{0}(p)\right\rangle, \\
F_{\mu} & =\left\langle\pi^{-}\left(p^{\prime}\right)\left|V_{\mu}^{\mathrm{w}}(x)\right| K^{0}(p)\right\rangle
\end{aligned}
$$

with the weak and electromagnetic currents defined in Eq. 4.23. The Ward identities $q^{\mu} V_{\mu \nu}=F_{\nu}$ and $q^{\mu} A_{\mu \nu}=0$ guarantee the gauge invariance of the total amplitude.

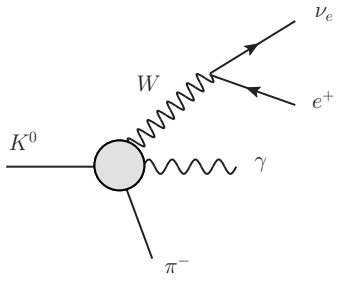

a)

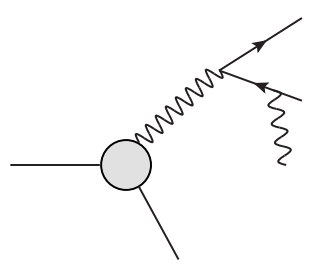

b)
FIG. 4 Diagrams describing the $K_{\ell 3 \gamma}^{0}$ amplitude.

The total amplitude can be decomposed into an "innerbremsstrahlung" (IB) and a "structure-dependent" (SD) part, both gauge invariant. IB captures the infrared singularities according to the Low (1958) theorem and the SD part contains terms of $\mathcal{O}(q)$ and higher. In Gasser et al. (2005) this decomposition was performed in such a way as to guarantee that the SD amplitude is regular in the Mandelstam plane, except for the branch points required by unitarity. In this treatment the tensor $V_{\mu \nu}$ has an IB component calculable in terms of $K_{\ell 3}$ form factors $f_{ \pm}$and a purely SD component, while the tensor $A_{\mu \nu}$ is purely SD. The SD amplitudes can be parametrized in terms of eight structure functions $V_{i}, A_{i}(i=1, \ldots, 4)$. Defining $W=p-p^{\prime}-q$, one has (Gasser et al. 2005):

$$
\begin{aligned}
A_{\mu \nu}^{\mathrm{SD}} & =i \epsilon_{\mu \nu \rho \sigma}\left(A_{1} p^{\rho} q^{\sigma}+A_{2} q^{\rho} W^{\sigma}\right) \\
& +i \epsilon_{\mu \lambda \rho \sigma} p^{\prime \lambda} q^{\rho} W^{\sigma}\left(A_{3} W_{\nu}+A_{4} p_{\nu}^{\prime}\right), \\
V_{\mu \nu}^{\mathrm{SD}} & =V_{1}\left(p_{\mu}^{\prime} q_{\nu}-g_{\mu \nu} p^{\prime} \cdot q\right)+V_{2}\left(W_{\mu} q_{\nu}-g_{\mu \nu} W \cdot q\right) \\
& +V_{3}\left(q \cdot W p_{\mu}^{\prime} W_{\nu}-p^{\prime} \cdot q W_{\mu} W_{\nu}\right) \\
& +V_{4}\left(q \cdot W p_{\mu}^{\prime} p_{\nu}^{\prime}-p^{\prime} \cdot q W_{\mu} p_{\nu}^{\prime}\right) .
\end{aligned}
$$

Early theoretical calculations of $K_{\ell 3 \gamma}$ were based on Low's theorem and current algebra (Fearing et al., 1970). Modern calculations (Bijnens et al. 1993 Gasser et al. 2005: Holstein, 1990, Kubis et al. 2007) have been performed within CHPT, which provides a natural framework to systematically expand the hadronic amplitudes $F_{\mu}, A_{\mu \nu}$, and $V_{\mu \nu}$. The chiral expansion for $V_{\mu \nu}$ contains both IB and SD terms and starts at $\mathcal{O}\left(p^{2}\right) . A_{\mu \nu}$ starts at $\mathcal{O}\left(p^{4}\right)$, with the leading contribution generated by the WZW functional (Wess and Zumino, 1971, Witten, 1983) accounting for the chiral anomaly.

The first complete analysis to $\mathcal{O}\left(p^{4}\right)$ was performed by Bijnens et al. (1993) who calculated the branching ratios for all $K_{\ell 3 \gamma}$ modes for given cuts in the photon energy and in the photon-electron opening angle in the kaon rest frame: $E_{\gamma}^{*}>E_{\gamma}^{\text {cut }}, \theta_{e \gamma}^{*}>\theta_{e \gamma}^{\text {cut }}$. Recently, the CHPT analysis was revisited and extended to $\mathcal{O}\left(p^{6}\right)$ for $K_{e 3 \gamma}^{0}$ (Gasser et al. 2005) and $K_{e 3 \gamma}^{ \pm}$decays (Kubis et al. 2007), which represent the theoretical state of the art. To $\mathcal{O}\left(p^{4}\right)$ the axial form factors $A_{i}$ are constant, while the vector form 
factors $V_{i}$ receive contributions from both LECs $\left(L_{9,10}^{r}\right)$ and loops. Since all cuts in loop functions lie far outside the physical region, the $V_{i}$ are constant to good accuracy. In order to gain control on the size of higher-order corrections, Gasser et al. (2005) and Kubis et al. (2007) performed a complete $p^{6}$ analysis of the axial terms $A_{i}$ (one loop) and determined the $\mathcal{O}\left(p^{6}\right) L_{i} \times L_{j}$ contributions to the vector terms $V_{i}$. Despite the appearance of cuts in the physical region, to $\mathcal{O}\left(p^{6}\right)$ the real parts of $V_{i}$ and $A_{i}$ are well approximated by smooth functions, with dominant uncertainties coming from the $\mathcal{O}\left(p^{6}\right)$ LECs.

The updated theoretical analysis (Gasser et al. 2005 . Kubis et al. 2007) leads to very stable predictions for the relative branching ratios defined by

$$
R_{K_{e 3 \gamma}}\left(E_{\gamma}^{\text {cut }}, \theta_{e \gamma}^{\text {cut }}\right) \equiv \frac{\Gamma_{K_{e 3 \gamma}}\left(E_{\gamma}^{*}>E_{\gamma}^{\text {cut }}, \theta_{e \gamma}^{*}>\theta_{e \gamma}^{\text {cut }}\right)}{\Gamma_{K_{e 3}}} .
$$

It turns out that this ratio is very insensitive to the details of the non-radiative $K_{\ell 3}$ form factor $f_{+}(t)$. Moreover, the SD terms contribute only a $\sim 1 \%$ correction to the IB result for $R$. The final predictions are (for representative cuts $^{6}$ for which experimental data are available):

$$
\begin{aligned}
& R_{K_{e 3 \gamma}^{L}}\left(E_{\gamma}^{\text {cut }}=30 \mathrm{MeV}, \theta_{e \gamma}^{\text {cut }}=20^{\circ}\right)=0.0096(1), \quad(4.68) \\
& R_{K_{e 3 \gamma}^{ \pm}}\left(E_{\gamma}^{\text {cut }}=10 \mathrm{MeV}, 26^{\circ}<\theta_{e \gamma}^{*}<53^{\circ}\right)=0.00559(6) .
\end{aligned}
$$

In order to measure the SD terms one needs to resort to differential decay distributions. A natural first observable to consider is the photon spectrum $d \Gamma / d E_{\gamma}^{*}$. It was found (Gasser et al., 2005; Kubis et al., 2007) that in both charged and neutral modes the SD terms correct the IB spectrum by one single function $f\left(E_{\gamma}^{*}\right)$ :

$$
\frac{d \Gamma}{d E_{\gamma}^{*}} \simeq \frac{d \Gamma_{\mathrm{IB}}}{d E_{\gamma}^{*}}+\langle X\rangle f\left(E_{\gamma}^{*}\right)
$$

where $\langle X\rangle$ is a mode-dependent linear combination of phase space averages of the SD terms $\left\langle V_{i}\right\rangle$ and $\left\langle A_{i}\right\rangle$. For the $K_{e 3 \gamma}^{0}$ mode, $\langle X\rangle=-1.2 \pm 0.4$ is dominated by $\left\langle V_{1}\right\rangle$. In contrast, in the $K_{e 3 \gamma}^{ \pm}$mode $\langle X\rangle=-2.2 \pm 0.7$ receives contributions of similar size from $\left\langle V_{1}\right\rangle$ and $\left\langle A_{1}\right\rangle$, thus making possible a detection of the effect of the chiral anomaly. More complicated angular distributions might be used to disentangle the dominant SD terms (Gasser et al., 2005, Kubis et al., 2007).

In parallel to new theoretical developments, there has been considerable experimental progress in these modes in the past decade. While we refer to Nakamura et al. (2010) for a complete experimental summary we focus here on the $K_{e 3 \gamma}$ decays. For the neutral mode, the world average is dominated by four recent measurements

\footnotetext{
${ }^{6}$ For more choices see Gasser et al. 2005) and Kubis et al. 2007).
}

(Alavi-Harati et al. 2001b; Alexopoulos et al., 2005: Ambrosino et al., 2008, Lai et al., 2005) that are not fully consistent (scale factor $S=1.9$ ):

$$
R_{K_{e 3 \gamma}^{L}}\left(E_{\gamma}^{\text {cut }}=30 \mathrm{MeV}, \theta_{e \gamma}^{\text {cut }}=20^{\circ}\right)=0.935(15) \times 10^{-2} .
$$

This PDG average (Nakamura et al. 2010) is about one sigma below the theoretical prediction given in Eq. 4.68). The most recent individual measurement from KLOE (Ambrosino et al. 2008),

$$
R_{K_{e 3 \gamma}^{L}}=\left(0.924 \pm 0.023_{\text {stat }} \pm 0.016_{\text {syst }}\right) \times 10^{-2},
$$

does not have sufficient precision to resolve the tension between the NA48 result $R=(0.964 \pm 0.013) \times 10^{-2}($ Lai et al. 2005) and the $\mathrm{KTeV}$ result $R=(0.916 \pm 0.017) \times$ $10^{-2}$ (Alexopoulos et al. 2005). By measuring the photon spectrum, the KLOE collaboration Ambrosino et al. 2008) performed a fit to the SD contribution $\langle X\rangle$, finding

$$
\langle X\rangle=-2.3 \pm 1.3_{\text {stat }} \pm 1.4_{\text {syst }},
$$

in agreement (within the large uncertainty) with the CHPT prediction $\langle X\rangle=-1.2 \pm 0.4$.

For the charged mode, the PDG average (Nakamura et al., 2010) is dominated by three measurements (Akimenko, 2007; Barmin et al., 1991; Bolotov et al., 1986), again not fully consistent (scale factor $S=1.3$ ):

$$
R_{K_{e 3 \gamma}^{ \pm}}\left(E_{\gamma}^{\text {cut }}=10 \mathrm{MeV}, 26^{\circ}<\theta_{e \gamma}^{*}<53^{\circ}\right)=0.00505(32) .
$$

This value is about one sigma below the theoretical prediction given in Eq. 4.69).

Despite the tremendous progress in $K_{\ell 3 \gamma}$ decays, from the comparison of theory and experiment we conclude that more accurate experimental data are desirable in order to perform definite tests of the CHPT predictions and to detect SD contributions.

\section{E. $K_{\ell 4}$}

$K_{\ell 4}$ is the shorthand notation for the decays

$$
\begin{aligned}
K^{+}(p) & \rightarrow \pi^{+}\left(p_{1}\right) \pi^{-}\left(p_{2}\right) \ell^{+}\left(p_{\ell}\right) \nu_{\ell}\left(p_{\nu}\right), \\
K^{+}(p) & \rightarrow \pi^{0}\left(p_{1}\right) \pi^{0}\left(p_{2}\right) \ell^{+}\left(p_{\ell}\right) \nu_{\ell}\left(p_{\nu}\right), \\
K^{0}(p) & \rightarrow \pi^{0}\left(p_{1}\right) \pi^{-}\left(p_{2}\right) \ell^{+}\left(p_{\ell}\right) \nu_{\ell}\left(p_{\nu}\right)
\end{aligned}
$$

and their charge-conjugate modes. In the isospin limit $\left(m_{u}=m_{d}, e=0\right)$, the amplitude for 4.75 is given by

$$
T=\frac{G_{F}}{\sqrt{2}} V_{u s}^{*} \bar{u}\left(p_{\nu}\right) \gamma^{\mu}\left(1-\gamma_{5}\right) v\left(p_{\ell}\right)\left(V_{\mu}-A_{\mu}\right),
$$

where the last factor contains the hadronic matrix elements of the strangeness-changing vector and axialvector currents,

$$
V_{\mu}-A_{\mu}=\left\langle\pi^{+}\left(p_{1}\right) \pi^{-}\left(p_{2}\right)\left|\bar{s} \gamma_{\mu} u-\bar{s} \gamma_{\mu} \gamma_{5} u\right| K^{+}(p)\right\rangle,
$$


with the form factor decompositions

$$
\begin{aligned}
V_{\mu} & =-\frac{H}{M_{K}^{3}} \varepsilon_{\mu \nu \rho \sigma}\left(p_{\ell}+p_{\nu}\right)^{\nu}\left(p_{1}+p_{2}\right)^{\rho}\left(p_{1}-p_{2}\right)^{\sigma}, \quad \text { (4.80) } \\
A_{\mu} & =-\frac{i}{M_{K}}\left[F\left(p_{1}+p_{2}\right)_{\mu}+G\left(p_{1}-p_{2}\right)_{\mu}+R\left(p_{\ell}+p_{\nu}\right)_{\mu}\right] .
\end{aligned}
$$

The matrix elements for the channels 4.76 and 4.77) can be obtained by isospin symmetry. The form factors $F, G, R$ and $H$ depend on the variables $s=\left(p_{1}+p_{2}\right)^{2}$, $t=\left(p_{1}-p\right)^{2}$ and $u=\left(p_{2}-p\right)^{2}$. Quite often,

$$
s=\left(p_{1}+p_{2}\right)^{2}, \quad s_{\ell}=\left(p_{\ell}+p_{\nu}\right)^{2}, \quad \cos \theta_{\pi}
$$

are used instead, where $\theta_{\pi}$ is the angle of the $\pi^{+}$in the center-of-mass system of the two charged pions relative to the dipion line of flight in the rest system of the kaon (Cabibbo and Maksymowicz, 1965, 1968).

The chiral expansion of the $K_{\ell 4}$ form factors was studied in CHPT at the one-loop level (Bijnens, 1990, Riggenbach et al., 1991) and beyond (Amorós et al. 2000a b. Bijnens et al. 1994). Comparison with experimental data yields information on several LECs.

The analysis of $K^{ \pm} \rightarrow \pi^{+} \pi^{-} e^{ \pm} \nu$ is a very efficient and clean approach to study pion-pion scattering at low energies. In the limit of isospin symmetry, one identifies the $\pi \pi$ phase shifts in the matrix element in a standard manner by performing a partial-wave expansion using unitarity and analyticity. Details of this procedure can be found in Pais and Treiman (1968a) and Berends et al. (1967, 1968). At the end, it boils down to the following parametrization of the form factors,

$$
\begin{aligned}
F & =F_{s} e^{i \delta_{s}}+F_{p} e^{i \delta_{p}} \cos \theta_{\pi}+\ldots, \\
G & =G_{p} e^{i \delta_{p}}+\ldots, \\
H & =H_{p} e^{i \delta_{p}}+\ldots,
\end{aligned}
$$

where the dots refer to neglected d-wave contributions. Note that the third axial form factor $R$ gets multiplied by a factor $m_{e}^{2} / s_{e}$ and cannot be measured in $K_{e 4}$ decays. One is therefore left with one phase difference $\delta=\delta_{s}-\delta_{p}$ and the four real form factors $F_{s}, F_{p}, G_{p}, H_{p}$.

Under the assumption of isospin symmetry, the form factors can be expanded in a series of the dimensionless invariants $q^{2}=s / 4 M_{\pi}^{2}-1$ and $s_{e} / 4 M_{\pi}^{2}$ Amorós and Bijnens, 1999). Within the currently available statistics (Batley et al. . 2010c), a constant term, two slope parameters and one curvature parameter are sufficient to describe the variation of the form factor $F_{s}$,

$$
F_{s}=f_{s}+f_{s}^{\prime} q^{2}+f_{s}^{\prime \prime} q^{4}+f_{e}^{\prime} \frac{s_{e}}{4 M_{\pi}^{2}},
$$

while two terms are needed to describe $G_{p}$,

$$
G_{p}=g_{p}+g_{p}^{\prime} q^{2} .
$$

$F_{p}$ and $H_{p}$ can be described by two constants. Based on an analysis of $1.13 \times 10^{6}$ decays, the NA48/2 collaboration reports the following results for the form factor measurements (Batley et al. 2010c):

$$
\begin{aligned}
f_{s}^{\prime} / f_{s} & =0.152 \pm 0.007 \pm 0.005, \\
f_{s}^{\prime \prime} / f_{s} & =-0.073 \pm 0.007 \pm 0.006 \\
f_{e}^{\prime} / f_{s} & =0.068 \pm 0.006 \pm 0.007 \\
f_{p} / f_{s} & =-0.048 \pm 0.003 \pm 0.004 \\
g_{p} / f_{s} & =0.868 \pm 0.010 \pm 0.010 \\
g_{p}^{\prime} / f_{s} & =0.089 \pm 0.017 \pm 0.013, \\
h_{p} / f_{s} & =-0.398 \pm 0.015 \pm 0.008,
\end{aligned}
$$

with statistical (first) and systematic (second) errors.

A preliminary branching ratio based on the NA48/2 data has recently been presented (Bloch-Devaux, 2011),

$$
\operatorname{BR}\left(K^{ \pm} \rightarrow \pi^{+} \pi^{-} e^{ \pm} \nu\right)=(4.279 \pm 0.035) \times 10^{-5},
$$

which improves by a factor of 3 the PDG average (4.09 \pm $0.10) \times 10^{-5}$ (Nakamura et al., 2010). The final analysis will allow to give precise absolute values for all decay form factors (Bloch-Devaux, 2011).

The extraction of the $\pi \pi$ scattering lengths from the phase shift measurements of $\delta=\delta_{s}-\delta_{p}$ requires additional theoretical ingredients. Forty years ago, Roy (1971) established an integral equation based on analyticity, unitarity and crossing that allows to predict the $\pi \pi$ phase values close to threshold by using experimental data above the matching point $(\sqrt{s}=0.8 \mathrm{GeV})$ and two subtraction constants $a_{0}$ and $a_{2}$, the $\mathrm{I}=0,2 \mathrm{~s}-$ wave scattering lengths. On the other hand, using measurements and the Roy equations, one can determine the corresponding values of the scattering lengths. $\mathrm{Nu}$ merical solutions of the Roy equations obtained by two groups (Ananthanarayan et al., 2001, Descotes-Genon et al. 2002) were employed for this purpose (Batley et al. 2010c).

Isospin-breaking turns out to be quite substantial in $K_{\ell 4}$ decays (Cuplov and Nehme, 2003, Nehme, 2004a b, 2005). Triggered by the precise results of NA48/2 (Batley et al. 2008b), a new theoretical procedure for the treatment of isospin-breaking on $K_{e 4}$ phase measurements was suggested by Colangelo et al. (2009). The measured phase of the $\mathrm{I}=0 \mathrm{~s}$-wave is no longer $\delta_{0}^{0}$ but

$$
\begin{aligned}
\psi_{0}= & \frac{1}{32 \pi F^{2}}\left[\left(4 \Delta_{\pi^{+} \pi^{0}}+s\right) \sigma_{ \pm}+\left(s-M_{\pi^{0}}^{2}\right)\left(1+\frac{3}{2 R}\right) \sigma_{0}\right] \\
& +\mathcal{O}\left(p^{4}\right) .
\end{aligned}
$$

In this expression, $F$ is the pion decay constant in the limit of chiral $\mathrm{SU}(2), \Delta_{\pi^{+} \pi^{0}}=M_{\pi^{ \pm}}^{2}-M_{\pi^{0}}^{2}, R$ is the quark-mass ratio defined in Eq. 44.11] and

$$
\sigma_{x}=\sqrt{1-4 M_{\pi^{x}}^{2} / s} \quad(x= \pm, 0) .
$$

Although the difference between the mass-symmetric angle $\left(\Delta_{\pi^{+} \pi^{0}}=0,1 / R=0, \sigma_{ \pm}=\sigma_{0}\right)$ and $\psi_{0}$ is rather small (10 to $15 \mathrm{mrad}$ ) over the whole range accessible in $K_{e 4}$ decays, there are non-negligible effects on the extraction of the scattering lengths (Batley et al., $2010 \mathrm{c}$ ), 


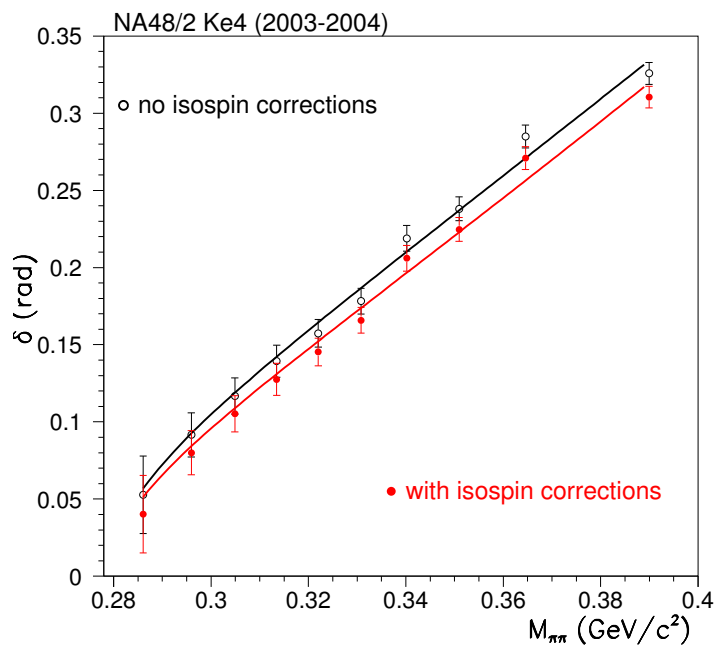

FIG. 5 Measurements of the phase shift $\delta$ without (open circles) and with (full circles) isospin mass effect corrections from NA48/2 $K_{e 4}$ data. From Batley et al. (2010c), Copyright CERN for the benefit of the NA48/2 Collaboration 2010.

as can be seen in Figs. 5 and 6 . Note that these effects had not been taken into account in the analyses of the older Geneva-Saclay (Rosselet et al., 1977) and BNL E865 (Pislak et al. , 2001, 2003) experiments.

A two-parameter fit of the NA48/2 data leads to the result (Batley et al., 2010c)

$$
\begin{aligned}
& a_{0}=0.2220 \pm 0.0128 \pm 0.0050 \pm 0.0037 \\
& a_{2}=-0.0432 \pm 0.0086 \pm 0.0034 \pm 0.0028
\end{aligned}
$$

where the errors refer to statistics, systematics and theory. Alternatively, using the additional theoretical constraint (Colangelo et al., 2000, 2001a b)

$$
\begin{aligned}
a_{2}= & -0.0444+0.236\left(a_{0}-0.220\right)-0.61\left(a_{0}-0.220\right)^{2} \\
& -9.9\left(a_{0}-0.220\right)^{3}
\end{aligned}
$$

(with a width of \pm 0.0008 ), a one-parameter fit was performed giving (Batley et al. $2010 \mathrm{c}$ )

$$
a_{0}=0.2206 \pm 0.0049 \pm 0.0018 \pm 0.0064,
$$

corresponding to the value $a_{2}=-0.0442$ from Eq. 4.90). These results should be compared with the most precise prediction to NNLO in CHPT (Colangelo et al. 2001a b):

$$
a_{0}=0.220 \pm 0.005, \quad a_{2}=-0.0444 \pm 0.0010 .
$$

A measurement of $K^{+} \rightarrow \pi^{0} \pi^{0} e^{+} \nu$ by the KEK-E740 Collaboration with a data sample of 216 events was published in Shimizu et al. (2004). However, due to large systematic errors, this result was not included in the PDG

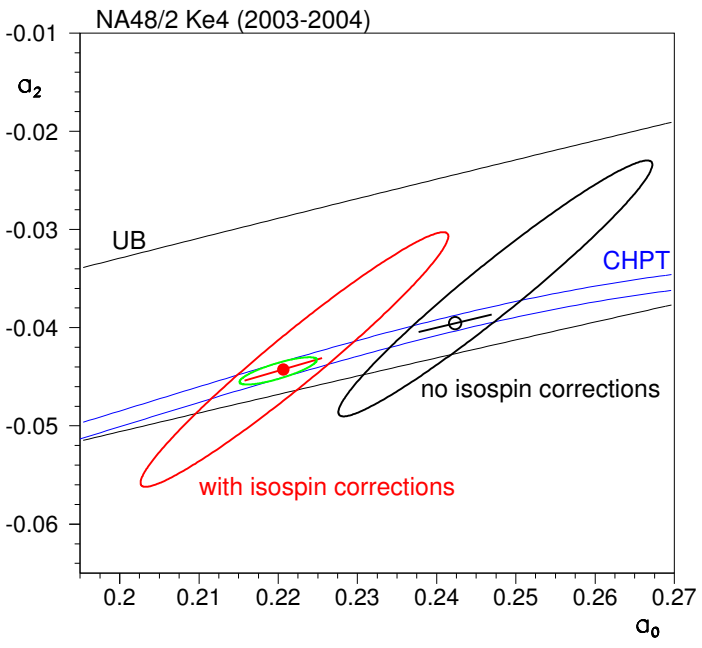

FIG. 6 Fits of the NA48/2 $K_{e 4}$ data in the $\left(a_{0}, a_{2}\right)$ plane without and with isospin mass effects. The wide band (UB) refers to the "universal band" [see Batley et al. (2010c) and Descotes-Genon et al. (2002) for details]. The large ellipses are $68 \%$ C.L. contours of the two-parameter fit leading to Eq. 4.89 and the circles the one-parameter fit of Eq. 4.91, imposing the CHPT constraint of Eq. 4.90). The small ellipse corresponds to Eq. 4.92. Adapted from Batley et al. (2010c).

fit $\operatorname{BR}\left(K^{+} \rightarrow \pi^{0} \pi^{0} e^{+} \nu\right)=(2.2 \pm 0.4) \times 10^{-5}$ (Nakamura et al., 2010), which uses data from an old low-statistics experiment (Barmin et al. 1 1988). Based on the analysis of $4.4 \times 10^{5} K_{e 4}^{\mathrm{VU}}$ events, the NA $48 / 2$ Collaboration has recently reported the preliminary branching ratio

$$
\operatorname{BR}\left(K^{ \pm} \rightarrow \pi^{0} \pi^{0} e^{ \pm} \nu\right)=(2.595 \pm 0.042) \times 10^{-5},
$$

which corresponds to a factor 10 improvement compared to the PDG value. They could also show the consistency of the $F_{s}$ form factors in the $K_{e 4}^{00}$ and $K_{e 4}^{+-}$modes.

Experimental results for $K_{L} \rightarrow \pi^{ \pm} \pi^{0} e^{\mp} \nu_{e}$ are also available (Batley et al. 2004a, Carroll et al., 1980: Makoff et al. 1993). Although the present statistics 5464 events in the experiment of Batley et al. (2004a)] is not comparable with the charged mode, the branching ratio and the form factors have been determined and a value for the chiral LEC $L_{3}$ was obtained:

$$
L_{3}=(-4.1 \pm 0.2) \times 10^{-3} \text {. }
$$

The contribution of the decay chain $K^{0} \rightarrow\left(K^{+} e^{-} \overline{\nu_{e}}\right) \rightarrow$ $\pi^{+} \pi^{0} e^{-} \overline{\nu_{e}}$ is suppressed by at least four orders of magnitude in the branching ratio (Chizhov, 2007). 
F. $K_{e 5}$

Except for the charge-conjugate modes, there are four kinematically allowed $K_{e 5}$ decays:

$$
\begin{gathered}
K^{+} \rightarrow \pi^{+} \pi^{-} \pi^{0} e^{+} \nu_{e}, \quad K^{+} \rightarrow \pi^{0} \pi^{0} \pi^{0} e^{+} \nu_{e} \\
K^{0} \rightarrow \pi^{0} \pi^{0} \pi^{-} e^{+} \nu_{e}, \quad K^{0} \rightarrow \pi^{+} \pi^{-} \pi^{-} e^{+} \nu_{e}
\end{gathered}
$$

related by the isospin relation (Blaser, 1995)

$$
\begin{aligned}
& 2 \Gamma\left(K^{+} \rightarrow \pi^{0} \pi^{0} \pi^{0} e^{+} \nu_{e}\right)= \\
& \Gamma\left(K_{L}^{0} \rightarrow \pi^{ \pm} \pi^{\mp} \pi^{ \pm} e^{\mp} \nu_{e}\right)-\Gamma\left(K_{L}^{0} \rightarrow \pi^{0} \pi^{0} \pi^{ \pm} e^{\mp} \nu_{e}\right) .
\end{aligned}
$$

At lowest order in CHPT, only the vector current contributes. Because of the small phase space, the branching ratios are suppressed: $\mathcal{O}\left(10^{-12}\right)$ for the $K^{+}$decays and $\mathcal{O}\left(10^{-11}\right)$ for the $K_{L}^{0}$ decays at leading order Blaser, 1995). Experimentally, there is only an upper bound (Nakamura et al., 2010)

$$
\mathrm{BR}\left(K^{+} \rightarrow \pi^{0} \pi^{0} \pi^{0} e^{+} \nu_{e}\right)<3.5 \times 10^{-6}
$$

\section{NONLEPTONIC DECAYS}

In this section, we first review the status of $K \rightarrow 2 \pi$ decays. NLO calculations including strong isospin breaking and electromagnetic corrections allow for the extraction of the lowest-order couplings $G_{8}, G_{27}$ in the nonleptonic weak Lagrangian 2.16 from the decay rates. A careful treatment of isospin violation is essential for a reliable determination of the s-wave $\pi \pi$ phase shift difference $\delta_{0}\left(M_{K}\right)-\delta_{2}\left(M_{K}\right)$. Despite a lot of efforts, the accuracy of theoretical predictions of the ratio $\epsilon^{\prime} / \epsilon$ still does not match the experimental precision. A NLO calculation of $K \rightarrow 3 \pi$ decays significantly improves the compatibility between theory and experiment even though some assumptions must be made about NLO LECs. Both isospin-violating and $\mathrm{CP}$-violating effects seem to be too small to be detected experimentally at this time. Somewhat unexpected, investigation of a cusp effect in the $M_{\pi^{0} \pi^{0}}$ distribution near threshold in $K \rightarrow 3 \pi$ decays has led to very precise values for the s-wave $\pi \pi$ scattering lengths.
A. $K \rightarrow \pi \pi$

The amplitudes for the two-pion decay modes can be parametrized in the form

$$
\begin{aligned}
A\left(K^{0} \rightarrow \pi^{+} \pi^{-}\right) & =A_{+-}=A_{1 / 2}+\frac{1}{\sqrt{2}}\left(A_{3 / 2}+A_{5 / 2}\right) \\
& =A_{0} e^{i \chi_{0}}+\frac{1}{\sqrt{2}} A_{2} e^{i \chi_{2}} \\
A\left(K^{0} \rightarrow \pi^{0} \pi^{0}\right) & =A_{00}=A_{1 / 2}-\sqrt{2}\left(A_{3 / 2}+A_{5 / 2}\right) \\
& =A_{0} e^{i \chi_{0}}-\sqrt{2} A_{2} e^{i \chi_{2}} \\
A\left(K^{+} \rightarrow \pi^{+} \pi^{0}\right) & =A_{+0}=\frac{3}{2}\left(A_{3 / 2}-\frac{2}{3} A_{5 / 2}\right) \\
& =\frac{3}{2} A_{2}^{+} e^{i \chi_{2}^{+}}
\end{aligned}
$$

This parametrization holds for the infrared-finite amplitudes where the Coulomb and infrared parts are removed from $A_{+-}, A_{+0}$ (Cirigliano et al. 2004a). The amplitudes $A_{\Delta I}(\Delta I=1 / 2,3 / 2,5 / 2)$ are generated by the $\Delta I$ component of the electroweak effective Hamiltonian in the limit of isospin conservation. In the SM, the $\Delta I=5 / 2$ piece is absent in the isospin limit. More precisely, $A_{5 / 2}=0$ in the absence of electromagnetic interactions and therefore $A_{2}=A_{2}^{+}$. In the limit of $\mathrm{CP}$ conservation, the amplitudes $A_{0}, A_{2}$ and $A_{2}^{+}$are real and positive by definition. In the isospin limit, the phases $\chi_{I}(I=0,2)$ can then be identified with the s-wave $\pi \pi$ scattering phase shifts $\delta_{I}\left(\sqrt{s}=M_{K}\right)$.

In the isospin limit (phase space is calculated with physical meson masses), the amplitudes $A_{0}, A_{2}$ and the phase difference $\chi_{0}-\chi_{2}$ can be obtained directly from the three $K \rightarrow \pi \pi$ branching ratios [we use the recent compilation of Antonelli et al. (2010a)]:

$$
\begin{aligned}
A_{0} & =(2.704 \pm 0.001) \times 10^{-7} \mathrm{GeV}, \\
A_{2} & =(1.210 \pm 0.002) \times 10^{-8} \mathrm{GeV}, \\
\chi_{0}-\chi_{2} & =(47.5 \pm 0.9)^{\circ} .
\end{aligned}
$$

In the presence of isospin violation and to lowest order in the chiral expansion, the amplitudes $A_{\Delta I}$ are given by (omitting isospin violation in the 27 -plet amplitudes)

$$
\begin{aligned}
A_{1 / 2}= & \frac{\sqrt{2}}{9} G_{27} F_{0}\left(M_{K^{0}}^{2}-M_{\pi^{0}}^{2}\right) \\
& +\sqrt{2} G_{8} F_{0}\left[\left(M_{K^{0}}^{2}-M_{\pi^{0}}^{2}\right)\left(1-\frac{2}{3 \sqrt{3}} \varepsilon^{(2)}\right)\right. \\
& \left.-\frac{2}{3} e^{2} F_{0}^{2}\left(g_{\text {ewk }}+2 Z\right)\right] \\
A_{3 / 2}= & \frac{10}{9} G_{27} F_{0}\left(M_{K^{0}}^{2}-M_{\pi^{0}}^{2}\right) \\
& +G_{8} F_{0}\left[\left(M_{K^{0}}^{2}-M_{\pi^{0}}^{2}\right) \frac{4}{3 \sqrt{3}} \varepsilon^{(2)}\right. \\
& \left.-\frac{2}{3} e^{2} F_{0}^{2}\left(g_{\mathrm{ewk}}+2 Z\right)\right] \\
A_{5 / 2}= & 0 .
\end{aligned}
$$


The various couplings are defined in Eqs. 2.16 and (2.18). Following Cirigliano et al. (2004a), we have expressed all amplitudes in terms of the neutral pion and kaon masses. The parameter $F_{0}$ can be identified with the pion decay constant $F_{\pi}$ at this order. The effect of strong isospin breaking is entirely due to the lowestorder $\pi^{0}-\eta$ mixing angle $\varepsilon^{(2)}$ given in Eq. 4.11. Electromagnetic interactions contribute through mass splitting (terms proportional to $Z$ ) and insertions of $g_{\text {ewk }}$. As a consequence of CPS symmetry (Bernard et al. 1985), electromagnetic corrections to the octet weak Hamiltonian do not generate a $\Delta I=5 / 2$ amplitude at lowest chiral order.

The NLO amplitudes in the isospin limit for both $K \rightarrow \pi \pi$ and $K \rightarrow 3 \pi$ were first calculated by Kambor et al. (1991). Their main conclusion was that the octet amplitude $A_{0}$ is strongly enhanced by final state interactions at NLO whereas the 27-plet amplitude $A_{2}$ is only mildly reduced. Since the generically small isospinviolating effects are enhanced in subdominant amplitudes with $\Delta I>1 / 2$ because of the $\Delta I=1 / 2$ rule, a systematic treatment of isospin violation is called for.

The first complete analysis of isospin breaking in $K \rightarrow \pi \pi$ amplitudes was carried out by Cirigliano et al. (2004a). References to earlier work can be found there. The analysis was repeated and extended to $K \rightarrow 3 \pi$ amplitudes by Bijnens and Borg (2005b). In the following, we update the analysis of Cirigliano et al. (2004a) with new experimental input (Antonelli et al. 2010a) and with new information on the LECs involved, both in the strong (Cirigliano et al., 2006) and in the electromagnetic sector (Ananthanarayan and Moussallam, 2004).

As usual at NLO in the chiral expansion, both oneloop diagrams with LO couplings and tree diagrams with a single insertion of NLO couplings must be taken into account. Including the leading isospin-breaking corrections (proportional to $G_{8}$ ), the amplitudes $A_{\Delta I}$ have the following form (Cirigliano et al. 2004a):

$$
\begin{aligned}
A_{\Delta I}= & G_{27} F_{\pi}\left(M_{K^{0}}^{2}-M_{\pi^{0}}^{2}\right) A_{\Delta I}^{(27)} \\
& +G_{8} F_{\pi}\left\{\left(M_{K^{0}}^{2}-M_{\pi^{0}}^{2}\right)\left[A_{\Delta I}^{(8)}+\varepsilon^{(2)} A_{\Delta I}^{(\varepsilon)}\right]\right. \\
& \left.-e^{2} F_{\pi}^{2}\left[A_{\Delta I}^{(\gamma)}+Z A_{\Delta I}^{(Z)}+g_{\mathrm{ewk}} A_{\Delta I}^{(g)}\right]\right\}
\end{aligned}
$$

The meaning of the amplitudes $A_{\Delta I}^{(X)}$ can be inferred from the superscript $X . A_{\Delta I}^{(8)}, A_{\Delta I}^{(27)}$ represent the octet and 27plet amplitudes in the isospin limit. $A_{\Delta I}^{(\varepsilon)}$ represents the effect of strong isospin breaking, while the electromagnetic contribution is split into a part induced by photon loops $A_{\Delta I}^{(\gamma)}$ and the parts induced by insertions of $Z$ and $g_{\text {ewk }}$ vertices $\left(A_{\Delta I}^{(Z)}\right.$ and $A_{\Delta I}^{(g)}$, respectively). The photon loops are actually infrared divergent: $A_{\Delta I}^{(\gamma)}$ are the infrared-finite (structure-dependent) terms whereas the divergent "infrared components" must be treated in combination with real photon emission. Details on this decomposition can be found in Cirigliano et al. (2004a).

Confronting the amplitudes in Eq. (5.4) with experimental rates (Antonelli et al. 2010a), one can extract the LO couplings $G_{8}, G_{27}$ and the phase difference $\chi_{0}-\chi_{2}$. Using instead the dimensionless couplings $g_{8}, g_{27}$ defined in Eq. (3.8), an update of the analysis of Cirigliano et al. (2004a) produces the results in Table IV.

\section{Comments.}

i. The reduction of the octet coupling $g_{8}$ at $\mathcal{O}\left(G_{F} p^{4}\right)$ corresponds to the enhancement of the $\Delta I=1 / 2$ amplitude by roughly $30 \%$ at NLO Kambor et al. 1991). The (rather generous) theory errors of $g_{8}$, $g_{27}$ at NLO account for the uncertainties of the various LECs involved. They should not be interpreted to include also effects of NNLO or higher. In fact, a calculation of the leading (double) chiral logarithms at NNLO (Büchler, 2006) confirmed that $\pi \pi$ rescattering generates an additional enhancement of $A_{0}$ at higher orders (Pallante and Pich, 2000, 2001). However, the values of $g_{8}, g_{27}$ displayed in Table IV are the appropriate values for nonleptonic weak amplitudes at LO or NLO, respectively.

ii. At NLO, both $g_{8}$ and $g_{27}$ receive small shifts from isospin-violating corrections. While this could be expected for $g_{8}$, it results from a cancellation of different effects in the case of $g_{27}$. On the other hand, as indicated in Table IV, the inclusion of isospin breaking reduces $g_{27}$ by roughly $10 \%$ at LO.

iii. The phase difference $\chi_{0}-\chi_{2}$ is taken as a fit parameter because the phases cannot be reliably calculated to NLO in CHPT. At NLO, the fitted phase difference is rather sensitive to isospin violation. The more interesting difference of s-wave $\pi \pi$ scattering phase shifts in the isospin limit $\delta_{0}-\delta_{2}$ at $\sqrt{s}=M_{K}$ will be discussed below.

iv. At NLO, electromagnetic corrections induce a $\Delta I=5 / 2$ amplitude:

$$
\begin{aligned}
f_{5 / 2} & \equiv \operatorname{Re} A_{2} / \operatorname{Re} A_{2}^{+}-1 \\
& =\left(8.44 \pm 0.02_{\exp } \pm 2.5_{\mathrm{th}}\right) \times 10^{-2}
\end{aligned}
$$

\section{1. $\pi \pi$ phase shifts from $K \rightarrow \pi \pi$ decays}

In the isospin limit, the phase difference $\chi_{0}-\chi_{2}$ accessible in $K \rightarrow \pi \pi$ decays equals the s-wave $\pi \pi$ phase shift difference $\delta_{0}\left(M_{K}\right)-\delta_{2}\left(M_{K}\right)$ (Watson's theorem). It has been a long-standing problem to reconcile the phase shift difference extracted from $K \rightarrow \pi \pi$ decays with other determinations of pion-pion phase shifts. This problem became especially acute after the precise determination of $\pi \pi$ phase shifts from combining dispersion theory with 
TABLE IV Weak couplings $g_{8}, g_{27}$ and phase difference $\chi_{0}-\chi_{2}$ at LO and NLO, with (IV) and without (IC) isospin violation. The theoretical uncertainties are twice the ones assigned by Cirigliano et al. (2004a).

\begin{tabular}{|c|c|c|c|c|}
\hline chiral order & isospin & $g_{8}$ & $g_{27}$ & $\chi_{0}-\chi_{2}$ \\
\hline LO & IC & 4.96 & 0.285 & $47.5^{\circ}$ \\
LO & IV & 4.99 & 0.253 & $47.8^{\circ}$ \\
NLO & IC & $3.62 \pm 0.002_{\exp } \pm 0.28_{\text {th }}$ & $0.286 \pm 0.0006_{\exp } \pm 0.028_{\text {th }}$ & $\left(47.5 \pm 0.9_{\exp }\right)^{\circ}$ \\
NLO & IV & $3.61 \pm 0.002_{\exp } \pm 0.28_{\text {th }}$ & $0.297 \pm 0.0006_{\exp } \pm 0.028_{\text {th }}$ & $\left(51.3 \pm 0.8_{\exp }\right)^{\circ}$ \\
\hline
\end{tabular}

CHPT (Colangelo et al. 2001a). We review here the present status of this problem (Cirigliano et al., 2009).

- The experimental situation has substantially improved in recent years for both the $K^{+}$and $K_{S}$ lifetimes and for the branching ratios of $K \rightarrow \pi \pi$ decays (Antonelli et al. 2010a). Compared to the analysis of Cirigliano et al. (2004a), the new experimental information reduces the phase shift difference by more than three degrees (with higher statistical significance), bringing it closer to the dispersion theoretical value (Cirigliano et al. 2008a).

- In the original analysis of Cirigliano et al. (2004a) the differences $\gamma_{I}=\chi_{I}-\delta_{I}\left(M_{K}\right)$ were calculated in CHPT although the $\chi_{I}$ and $\delta_{I}$ separately come out much too small at NLO in the chiral expansion. The analysis can be improved by relying to a lesser extent on the NLO calculation of $K \rightarrow \pi \pi$ amplitudes altogether. Using less information potentially increases the uncertainty but this is compensated by a less biased comparison with the data. The resulting estimate of isospin violation is more robust and it leads to a further decrease of the phase shift difference by nearly two degrees.

The main idea of the alternative procedure of Cirigliano et al. (2009) is to use only the isospin-violating parts of the NLO amplitudes as theory input and to determine $\delta_{0}\left(M_{K}\right)-\delta_{2}\left(M_{K}\right)$ directly from the data. In contrast to the chiral corrections for the full amplitudes, the isospin-violating (IV) corrections are much smaller and therefore more suitable for a perturbative estimate.

The amplitudes are now parametrized as

$$
\begin{aligned}
A_{+-} & =\bar{A}_{0} e^{i \delta_{0}\left(M_{K}\right)}+\frac{1}{\sqrt{2}} \bar{A}_{2} e^{i \delta_{2}\left(M_{K}\right)}+\Delta A_{+-}^{\mathrm{IV}}, \\
A_{00} & =\bar{A}_{0} e^{i \delta_{0}\left(M_{K}\right)}-\sqrt{2} \bar{A}_{2} e^{i \delta_{2}\left(M_{K}\right)}+\Delta A_{00}^{\mathrm{IV}} \\
A_{+0} & =\frac{3}{2} \bar{A}_{2} e^{i \delta_{2}\left(M_{K}\right)}+\Delta A_{+0}^{\mathrm{IV}}
\end{aligned}
$$

All isospin violation is contained in $\Delta A_{+-}^{\mathrm{IV}}, \Delta A_{00}^{\mathrm{IV}}, \Delta A_{+0}^{\mathrm{IV}}$ that can be extracted from the NLO amplitudes of Cirigliano et al. (2004a). Since isospin violation was neglected in the 27-plet amplitudes in view of the $\Delta I=1 / 2$ rule, $\Delta A_{n}^{\mathrm{IV}}(n=+-, 00,+0)$ scale linearly with the lowest-order octet coupling $g_{8}$.

The moduli of the amplitudes in the isospin limit are denoted as $\bar{A}_{0}, \bar{A}_{2}$. These amplitudes together with the phase shift difference $\delta_{0}\left(M_{K}\right)-\delta_{2}\left(M_{K}\right)$ are then determined directly from the rates. For this purpose, the moduli are written as

$$
\begin{aligned}
\left|A_{+-}\right|= & \mid \bar{A}_{0}+\frac{1}{\sqrt{2}} \bar{A}_{2} e^{i\left(\delta_{2}\left(M_{K}\right)-\delta_{0}\left(M_{K}\right)\right)} \\
& +\Delta A_{+-}^{\mathrm{IV}} e^{-i \delta_{0}\left(M_{K}\right)} \mid \\
\left|A_{00}\right|= & \mid \bar{A}_{0}-\sqrt{2} \bar{A}_{2} e^{i\left(\delta_{2}\left(M_{K}\right)-\delta_{0}\left(M_{K}\right)\right)} \\
& +\Delta A_{00}^{\mathrm{IV}} e^{-i \delta_{0}\left(M_{K}\right)} \mid, \\
\left|A_{+0}\right|= & \left|\frac{3}{2} \bar{A}_{2} e^{i\left(\delta_{2}\left(M_{K}\right)-\delta_{0}\left(M_{K}\right)\right)}+\Delta A_{+0}^{\mathrm{IV}} e^{-i \delta_{0}\left(M_{K}\right)}\right| .
\end{aligned}
$$

In order to determine $\bar{A}_{0}, \bar{A}_{2}$ and $\delta_{0}\left(M_{K}\right)-\delta_{2}\left(M_{K}\right)$ from the three rates, also the $I=0$ phase $\delta_{0}\left(M_{K}\right)$ is needed as input (Colangelo et al., 2001a):

$$
\delta_{0}\left(M_{K}\right)=(39.2 \pm 1.5)^{\circ} .
$$

From the structure of the moduli in Eq. (5.7) it is obvious that the precise value of $\delta_{0}\left(M_{K}\right)$ has little impact on the phase shift difference.

With the (updated) values of $\Delta A_{n}^{\mathrm{IV}}$ (Cirigliano et al. 2004a) and with $g_{8}=3.6$ (see Table IV], the experimental rates (Antonelli et al. 2010a) give rise to

$$
\begin{gathered}
\bar{A}_{0}=(2.7030 \pm 0.0008) \times 10^{-7} \mathrm{GeV}, \\
\bar{A}_{2}=(0.1249 \pm 0.0003) \times 10^{-7} \mathrm{GeV}, \\
\delta_{0}\left(M_{K}\right)-\delta_{2}\left(M_{K}\right)=(52.54 \pm 0.83)^{\circ},
\end{gathered}
$$

where the errors are purely experimental. The octet enhancement in $K \rightarrow \pi \pi$ decays is characterized by the amplitude ratio

$$
\frac{\bar{A}_{0}}{\bar{A}_{2}}=21.63 \pm 0.04,
$$

again with experimental error only.

There are various sources of theoretical uncertainties associated with the phase shift difference. Whereas the 
error of $\delta_{0}$ in Eq. 5.8 is completely negligible, a generous error of $20 \%$ for the overall scale $g_{8}$ of the isospinviolating amplitudes gives rise to an uncertainty $\pm 1.1^{\circ}$ for the phase difference. The major part of the error is due to unknown effects of $\mathcal{O}\left(e^{2} p^{4}\right)$ that were estimated by Cirigliano et al. (2009) in two different ways. Altogether, the final value for the phase shift difference in the isospin limit from $K \rightarrow \pi \pi$ decays is

$$
\begin{aligned}
{\left[\delta_{0}\left(M_{K}\right)\right.} & \left.-\delta_{2}\left(M_{K}\right)\right]_{K \rightarrow \pi \pi} \\
& =\left(52.5 \pm 0.8_{\exp } \pm 2.8_{\mathrm{th}}\right)^{\circ}
\end{aligned}
$$

Before comparing this result with other determinations, one should recall that the isospin limit is defined in terms of the neutral meson masses. However, a LO estimate (Cirigliano et al. 2009) suggests that expanding instead around the charged pion mass modifies the phase shift difference only by much less than a degree.

The most recent determinations of the phase shift difference from $\pi \pi$ scattering data are

$$
\begin{aligned}
& {\left[\delta_{0}\left(M_{K}\right)-\delta_{2}\left(M_{K}\right)\right]_{\pi \pi}} \\
& \quad=\left\{\begin{array}{cc}
(47.7 \pm 1.5)^{\circ} & \text { (Colangelo et al. 2001a), } \\
(50.9 \pm 1.2)^{\circ} & \text { (Kamiński et al. 2008), } \\
(47.7 \pm 0.4)^{\circ} & \text { (Batley et al. 2010c), } \\
(47.3 \pm 0.9)^{\circ} & \text { García-Martín et al. 2011). }
\end{array}\right.
\end{aligned}
$$

These values would agree perfectly well with the phase difference $\chi_{0}-\chi_{2}$ in Eq. $(5.2)$, obtained in the isospin limit. However, due to the large ratio $A_{0} / A_{2}$ in $K \rightarrow$ $2 \pi$ decays, isospin-breaking corrections to the dominant $\Delta I=1 / 2$ amplitude generate sizable contributions to $A_{2}$, modifying also the amplitude phases. The updated determination of the phase shift difference from $K \rightarrow$ $2 \pi$ decays in Eq. 5.11) Cirigliano et al., 2009) turns out to be in reasonable agreement with the $\pi \pi$ results in Eq. (5.12), although with a larger uncertainty.

\section{2. $\epsilon^{\prime} / \epsilon$}

The CP-violating ratio $\epsilon^{\prime} / \epsilon$ constitutes a fundamental test for our understanding of flavor-changing phenomena. $\epsilon$ and $\epsilon^{\prime}$ parametrize different sources of CP violation in $K_{L} \rightarrow \pi \pi$ :

$$
\begin{gathered}
\eta_{+-} \equiv \frac{A\left(K_{L} \rightarrow \pi^{+} \pi^{-}\right)}{A\left(K_{S} \rightarrow \pi^{+} \pi^{-}\right)}=\epsilon+\epsilon^{\prime} \\
\eta_{00} \equiv \frac{A\left(K_{L} \rightarrow \pi^{0} \pi^{0}\right)}{A\left(K_{S} \rightarrow \pi^{0} \pi^{0}\right)}=\epsilon-2 \epsilon^{\prime} .
\end{gathered}
$$

The dominant effect from CP violation in $K^{0}-\overline{K^{0}}$ mixing is contained in $\epsilon$, while $\epsilon^{\prime}$ accounts for direct $\mathrm{CP}$ violation in the decay amplitudes. The present experimental world average (Abouzaid et al. 2011a; Alavi-Harati et al., 1999, 2003a; Barr et al. 1993, Batley et al. 2002; Burkhardt et al., 1988, Fanti et al., 1999, Gibbons et al., 1993: Lai et al. 2001),

$$
\operatorname{Re}\left(\epsilon^{\prime} / \epsilon\right)=\frac{1}{3}\left(1-\left|\frac{\eta_{00}}{\eta_{+-}}\right|\right)=(16.8 \pm 1.4) \times 10^{-4}
$$

demonstrates the existence of direct $\mathrm{CP}$ violation in $\mathrm{K}$ decays.

When CP violation is turned on, the amplitudes $A_{0}$, $A_{2}, A_{2}^{+}$acquire imaginary parts. To first order in $\mathrm{CP}$ violation, $\epsilon^{\prime}$ is given by

$$
\epsilon^{\prime}=-\frac{i}{\sqrt{2}} e^{i\left(\chi_{2}-\chi_{0}\right)} \frac{\operatorname{Re} A_{2}}{\operatorname{Re} A_{0}}\left[\frac{\operatorname{Im} A_{0}}{\operatorname{Re} A_{0}}-\frac{\operatorname{Im} A_{2}}{\operatorname{Re} A_{2}}\right] .
$$

Since $\operatorname{Im} A_{I}$ is $\mathrm{CP}$-odd the quantities $\operatorname{Re} A_{I}$ and $\chi_{I}$ are only needed in the CP limit $(I=0,2)$. $\epsilon^{\prime}$ is suppressed by the small ratio $\operatorname{Re} A_{2} / \operatorname{Re} A_{0} \approx 1 / 22$. The phase $\phi_{\epsilon}^{\prime}=$ $\chi_{2}-\chi_{0}+\pi / 2=(42.5 \pm 0.9)^{\circ}$ is very close to the so-called superweak phase (Nakamura et al., 2010)

$$
\phi_{\epsilon} \approx \tan ^{-1}\left(\frac{2\left(M_{K_{L}}-M_{K_{S}}\right)}{\Gamma_{K_{S}}-\Gamma_{K_{L}}}\right)=(43.51 \pm 0.05)^{\circ},
$$

implying that $\cos \left(\phi_{\epsilon}^{\prime}-\phi_{\epsilon}\right) \approx 1$.

To obtain the theoretical SM prediction for $\epsilon^{\prime}$, the $\mathrm{CP}$-conserving amplitudes $\operatorname{Re} A_{I}$ are set to their experimentally determined values. This procedure avoids the large uncertainties associated with the hadronic matrix elements of the four-quark operators in $\mathcal{L}_{\text {eff }}^{\Delta S=1}$, in particular $\operatorname{Re} A_{0}$ that involves several octet operators with a complicated mixing under the renormalization group. Thus, one only needs a first-principle calculation of the CP-odd amplitudes $\operatorname{Im} A_{0}$ and $\operatorname{Im} A_{2}$; the first one is completely dominated by the strong penguin operator $Q_{6}$, while the leading contribution to the second one comes from the electromagnetic penguin $Q_{8}$. Fortunately, those are precisely the operators that are expected to be better approximated through our large- $N_{C}$ estimate of LECs.

Equation (5.15) involves a delicate balance between the two isospin contributions. A naive estimate of $\operatorname{Im} A_{I}$ at lowest order in the chiral expansion, i.e., using the tree-level formulae in Eq. (5.3), results in a large numerical cancellation leading to unrealistically low values of $\epsilon^{\prime} / \epsilon$ around $7 \times 10^{-4}$ (Bosch et al. 2000 ; Buchalla et al. 1996; Buras et al. 2001; Buras and Jamin, 2004, Ciuchini et al. $1999,1993,1994,1998)$. The true SM prediction is then very sensitive to the precise values of the two contributing amplitudes (Bertolini et al., 2000; Hambye et al. 2000). The one-loop CHPT corrections generate an important enhancement $(\sim 35 \%)$ of the isoscalar amplitude ( $\pi \pi$ rescattering) and a reduction of $A_{2}$, destroying the accidental lowest-order cancellation and bringing the SM prediction of $\epsilon^{\prime} / \epsilon$ in good agreement with the experimental measurement (Pallante and Pich, 2000, 2001, Pallante et al. 2001).

Owing to the large ratio $\operatorname{Re} A_{0} / \operatorname{Re} A_{2}$, isospin violation plays also an important role in $\epsilon^{\prime} / \epsilon$. Small IV corrections proportional to the large octet coupling $G_{8}$ feed into the 
small amplitude $A_{2}$ generating relatively large contributions, which can modify the predicted value of $\epsilon^{\prime}$ in a sizable way. A systematic analysis of isospin-breaking corrections in $\epsilon^{\prime}$ was undertaken in Cirigliano et al. (2003c) where references to earlier work can be found. To first order in isospin violation, one finds

$\epsilon^{\prime}=-\frac{i}{\sqrt{2}} e^{i\left(\chi_{2}-\chi_{0}\right)} \omega_{+}\left[\frac{\operatorname{Im} A_{0}^{(0)}}{\operatorname{Re} A_{0}^{(0)}}\left(1+\Delta_{0}+f_{5 / 2}\right)-\frac{\operatorname{Im} A_{2}}{\operatorname{Re} A_{2}^{(0)}}\right]$

where

$$
\begin{aligned}
& \omega_{+}=\operatorname{Re} A_{2}^{+} / \operatorname{Re} A_{0}, \\
& \Delta_{0}=\frac{\operatorname{Im} A_{0}}{\operatorname{Im} A_{0}^{(0)}} \frac{\operatorname{Re} A_{0}^{(0)}}{\operatorname{Re} A_{0}}-1
\end{aligned}
$$

and $f_{5 / 2}$ is defined in Eq. (5.5). The superscript (0) on the amplitudes denotes the isospin limit.

$\operatorname{Im} A_{2}$ is itself first order in isospin breaking. One usually separates the electromagnetic penguin contribution to $\operatorname{Im} A_{2}$ from the isospin-breaking effects generated by other four-quark operators:

$$
\operatorname{Im} A_{2}=\operatorname{Im} A_{2}^{\mathrm{emp}}+\operatorname{Im} A_{2}^{\text {non-emp }} .
$$

A discussion of this scheme-dependent separation in the framework of CHPT can be found in Cirigliano et al. (2003c). Splitting off the electromagnetic penguin contribution to $\operatorname{Im} A_{2}$ in this way, one can write $\epsilon^{\prime}$ in a more familiar form as

$$
\epsilon^{\prime}=-\frac{i}{\sqrt{2}} e^{i\left(\chi_{2}-\chi_{0}\right)} \omega_{+}\left[\frac{\operatorname{Im} A_{0}^{(0)}}{\operatorname{Re} A_{0}^{(0)}}\left(1-\Omega_{\mathrm{eff}}\right)-\frac{\operatorname{Im} A_{2}^{\mathrm{emp}}}{\operatorname{Re} A_{2}^{(0)}}\right],
$$

where

$$
\begin{aligned}
\Omega_{\mathrm{eff}} & =\Omega_{\mathrm{IV}}-\Delta_{0}-f_{5 / 2} \\
\Omega_{\mathrm{IV}} & =\frac{\operatorname{Re} A_{0}^{(0)}}{\operatorname{Re} A_{2}^{(0)}} \times \frac{\operatorname{Im} A_{2}^{\mathrm{non}-\mathrm{emp}}}{\operatorname{Im} A_{0}^{(0)}} .
\end{aligned}
$$

The quantity $\Omega_{\text {eff }}$ includes all effects to leading order in isospin breaking and it generalizes the more traditional parameter $\Omega_{\mathrm{IV}}$. Although $\Omega_{\mathrm{IV}}$ is in principle enhanced by the large ratio $\operatorname{Re} A_{0}^{(0)} / \operatorname{Re} A_{2}^{(0)}$, the actual numerical analysis shows all three terms in Eq. (5.21) to be relevant when both strong and electromagnetic isospin violation are included (Cirigliano et al. 2003c).

The numerical analysis of Cirigliano et al. (2003c) found large cancellations among the different contributions to $\Omega_{\text {eff }}$. A well-known example are the contributions of strong isospin violation via $\pi^{0}-\eta$ mixing where the sum of $\eta$ and $\eta^{\prime}$ exchange generates an $\Omega_{\mathrm{IV}}$ of the order of $25 \%$. However, already at the level of $\pi^{0}-\eta$ mixing alone, a complete NLO calculation (Ecker et al. 2000b) produces a destructive interference in $\Omega_{\mathrm{IV}}$, with $\Omega_{\mathrm{IV}}=(15.9 \pm 4.5) \times 10^{-2}$. Inclusion of electromagnetic effects slightly increases $\Omega_{\mathrm{IV}}$ and generates sizable $\Delta_{0}$ and $f_{5 / 2}$, which interfere destructively with $\Omega_{\mathrm{IV}}$ to produce the final result (Cirigliano et al. 2003c):

$$
\Omega_{\mathrm{eff}}=(6.0 \pm 7.7) \times 10^{-2} .
$$

The small value obtained for $\Omega_{\text {eff }}$ reinforces the dominance of the gluonic penguin operator $Q_{6}$ in $\epsilon^{\prime}$. Taking this into account and updating all other inputs, the SM prediction for $\epsilon^{\prime} / \epsilon$ turns out to be (Pallante et al. 2001; Pich, 2004)

$$
\operatorname{Re}\left(\epsilon^{\prime} / \epsilon\right)=\left(19 \pm 2_{-6}^{+9} \pm 6\right) \times 10^{-4},
$$

in excellent agreement with the experimental measurement shown in Eq. (5.14). The first error was estimated by varying the renormalization scale $\mu$ between $M_{\rho}$ and $m_{c}$. The uncertainty induced by $m_{s}$, which was taken in the range $m_{s}(2 \mathrm{GeV})=110 \pm 20 \mathrm{MeV}$, is indicated by the second error.

The most critical step is the matching between the short- and long-distance descriptions, which was done at leading order in $1 / N_{C}$. Since all next-to-leading ultraviolet (OPE) and infrared (CHPT) logarithms have been taken into account, our educated guess for the theoretical uncertainty associated with subleading contributions is $\sim 30 \%$ (third error).

The control of non-logarithmic corrections at NLO in $1 / N_{C}$ remains a challenge for future investigations. Several dispersive analyses (Bijnens et al., 2001, 2004; Cirigliano et al., 2001, 2003a: Cirigliano and Golowich, 2000, 2002, Donoghue and Golowich, 2000; Knecht et al. 1999b, 2001; Narison, 2001) and lattice calculations (Blum et al., 2003; Boucaud et al., 2005, Noaki et al., 2003) of $\left\langle Q_{8}\right\rangle \equiv\left\langle 2 \pi\left|Q_{8}\right| K\right\rangle$ already exist (most of them in the chiral limit). Taking the chiral corrections into account, those results are compatible with the value used in Eq. (5.24). Unfortunately, the hadronic matrix element $\left\langle Q_{6}\right\rangle \equiv\left\langle 2 \pi\left|Q_{6}\right| K\right\rangle$ is more difficult to compute. Two analytical estimates in the chiral limit, using the so-called minimal hadronic approximation (Hambye et al., 2003 ) and the X-boson approach (Bijnens and Prades, 1999. 2000a), find large $1 / N_{C}$ corrections to $\left\langle Q_{6}\right\rangle$. It would be interesting to understand the physics behind those contributions and to study whether corrections of similar size are present for physical values of the quark masses.

Lattice calculations of $\left\langle Q_{6}\right\rangle$ are still not very reliable and give contradictory results, often with the wrong sign (Bhattacharya et al., 2005, Blum et al. 2003, Noaki et al., 2003 Pekurovsky and Kilcup 2001). The usual procedure in lattice simulations has been to calculate $K \rightarrow \pi$ and $K \rightarrow$ vacuum matrix elements and use lowest-order CHPT to recover the physical $K \rightarrow 2 \pi$ amplitudes. In addition to all usual lattice artifacts (finite volume and lattice spacing, quenched approximation, unphysical masses, etc.), this misses completely the crucial role of final-state interactions in the isoscalar octet amplitude. Thus, a truly major effort is needed to compute the $K \rightarrow 2 \pi$ matrix elements directly. Some progress in this direction has been achieved, relating the physical $K \rightarrow 2 \pi$ amplitudes to the corresponding matrix 
elements in a finite Euclidean volume, which are better suited for lattice simulations (Lellouch and Lüscher, 2001, Lin et al. 2001). So far, this technique has been implemented in simulations of the $\Delta I=3 / 2 \quad K^{+} \rightarrow \pi^{+} \pi^{0}$ amplitude with promising results (Boucaud et al. 2005 , Goode and Lightman, 2010, Kim and Sachrajda, 2010, Liu, 2010). A first estimate of the $A_{0}$ amplitude at unphysical kinematics has been recently reported (Blum et al. 2011).

More work is needed to reduce the present uncertainty quoted in Eq. (5.24). This is a difficult task, but progress in this direction may be expected in the next few years.

\section{3. $\mathrm{CP}$ violation in $K^{0}-\overline{K^{0}}$ mixing}

Since $\operatorname{Re}\left(\epsilon^{\prime} / \epsilon\right) \ll 1$, the ratios $\eta_{+-}$and $\eta_{00}$ provide a direct measurement of $|\epsilon|$ (Nakamura et al., 2010):

$$
|\epsilon|=\frac{1}{3}\left(2\left|\eta_{+-}\right|+\left|\eta_{\text {oo }}\right|\right)=(2.228 \pm 0.011) \times 10^{-3},
$$

in perfect agreement with the semileptonic asymmetry

$$
\begin{gathered}
\frac{\Gamma\left(K_{L} \rightarrow \pi^{-} \ell^{+} \nu\right)-\Gamma\left(K_{L} \rightarrow \pi^{+} \ell^{-} \bar{\nu}\right)}{\Gamma\left(K_{L} \rightarrow \pi^{-} \ell^{+} \nu\right)+\Gamma\left(K_{L} \rightarrow \pi^{+} \ell^{-} \bar{\nu}\right)}=\frac{2 \operatorname{Re}(\epsilon)}{1+|\epsilon|^{2}} \\
=(3.32 \pm 0.06) \times 10^{-3}
\end{gathered}
$$

The theoretical prediction can be written in the form (Buchalla et al. 1996, Buras and Guadagnoli, 2008)

$$
\begin{aligned}
|\epsilon|=C_{\epsilon} k_{\epsilon} \hat{B}_{K} A^{2} \lambda^{6} \bar{\eta} \times & \left\{A^{2} \lambda^{4}(1-\bar{\rho}) \eta_{t t} S_{0}\left(x_{t}\right)\right. \\
& \left.+\eta_{c t} S_{0}\left(x_{c}, x_{t}\right)-\eta_{c c} S_{0}\left(x_{c}\right)\right\}
\end{aligned}
$$

where $x_{i}=m_{i}^{2} / M_{W}^{2}, S_{0}\left(x_{i}, x_{j}\right)$ and $S_{0}\left(x_{i}\right)$ are the Inami and Lim (1981) box functions,

$$
C_{\epsilon}=\frac{G_{F}^{2} F_{K}^{2} M_{K^{0}} M_{W}^{2}}{3 \sqrt{2} \pi^{2}\left(M_{K_{L}}-M_{K_{S}}\right)}=3.7 \times 10^{4},
$$

$\eta_{t t}^{\mathrm{NLO}}=0.5765 \pm 0.0065, \eta_{c c}^{\mathrm{NLO}}=1.43 \pm 0.23$ and $\eta_{c t}^{\mathrm{NNLO}}=$ $0.496 \pm 0.047$ are short-distance QCD corrections (Brod and Gorbahn, 2010, Buras et al., 1990, Herrlich and Nierste, 1996) and $k_{\epsilon}=0.94 \pm 0.02$ accounts for small longdistance contributions (Buras et al., 2010).

The renormalization-group-invariant parameter $\hat{B}$ measures the hadronic matrix element $\left\langle\bar{K}^{0}\left|Q_{\Delta S=2}\right| K^{0}\right\rangle$ in units of its vacuum saturation approximation. In the large $-N_{C}$ limit, $\hat{B}=3 / 4$ (Buras and Gérard, 1986 . Gaiser et al. (1981). The most precise lattice determinations, obtained with $2+1$ active flavors, quote $\hat{B}=$ $0.724 \pm 0.030$ (Aubin et al. 2010) and $\hat{B}=0.749 \pm 0.027$ (Aoki et al. 2011).

Eq. (5.27) provides a parabolic constraint in the plane $\bar{\rho}-\bar{\eta}$ that is included in the SM unitarity triangle fits (Bona et al. 2006, Charles et al., 2005). The recent precise lattice value for $\hat{B}$ introduces some tension in the fit. Determining $\bar{\eta}$ and $\bar{\rho}$ from other observables, it implies $|\epsilon|=(1.90 \pm 0.26) \times 10^{-3}$ (Brod and Gorbahn, 2010), slightly smaller than (5.25).
B. $K \rightarrow 3 \pi$

There are five CP-conserving decays to three pions (the $K^{-}$decays are not listed separately):

$$
\begin{aligned}
A_{000}^{L} & =A\left(K_{L}(k) \rightarrow \pi^{0}\left(p_{1}\right) \pi^{0}\left(p_{2}\right) \pi^{0}\left(p_{3}\right)\right), \\
A_{+-0}^{L} & =A\left(K_{L}(k) \rightarrow \pi^{+}\left(p_{1}\right) \pi^{-}\left(p_{2}\right) \pi^{0}\left(p_{3}\right)\right), \\
A_{+-0}^{S} & =A\left(K_{S}(k) \rightarrow \pi^{+}\left(p_{1}\right) \pi^{-}\left(p_{2}\right) \pi^{0}\left(p_{3}\right)\right), \\
A_{00+} & =A\left(K^{+}(k) \rightarrow \pi^{0}\left(p_{1}\right) \pi^{0}\left(p_{2}\right) \pi^{+}\left(p_{3}\right)\right), \\
A_{++-} & =A\left(K^{+}(k) \rightarrow \pi^{+}\left(p_{1}\right) \pi^{+}\left(p_{2}\right) \pi^{-}\left(p_{3}\right)\right) .
\end{aligned}
$$

For the kinematics one uses the variables

$$
s_{1}=\left(k-p_{1}\right)^{2}, s_{2}=\left(k-p_{2}\right)^{2}, s_{3}=\left(k-p_{3}\right)^{2}
$$

with

$$
\begin{aligned}
s_{0} & =\frac{1}{3}\left(s_{1}+s_{2}+s_{3}\right) \\
& =\frac{1}{3}\left(M_{K}^{2}+M_{\pi^{1}}^{2}+M_{\pi^{2}}^{2}+M_{\pi^{3}}^{2}\right),
\end{aligned}
$$

where the masses are those of the particles appearing in the decay under consideration.

In terms of the Dalitz plot variables

$$
x=\frac{s_{2}-s_{1}}{M_{\pi^{+}}^{2}}, \quad y=\frac{s_{3}-s_{0}}{M_{\pi^{+}}^{2}},
$$

the amplitudes are often expanded as (Devlin and Dickey, 1979)

$$
\begin{aligned}
A_{000}^{L}= & 3\left(\alpha_{1}+\alpha_{3}\right)+3\left(\zeta_{1}-2 \zeta_{3}\right)\left(y^{2}+\frac{1}{3} x^{2}\right) \\
A_{+-0}^{L}= & \left(\alpha_{1}+\alpha_{3}\right)-\left(\beta_{1}+\beta_{3}\right) y \\
& +\left(\zeta_{1}-2 \zeta_{3}\right)\left(y^{2}+\frac{1}{3} x^{2}\right) \\
& +\left(\xi_{1}-2 \xi_{3}\right)\left(y^{2}-\frac{1}{3} x^{2}\right) \\
A_{+-0}^{S}= & \frac{2}{3} \sqrt{3} \gamma_{3} x-\frac{4}{3} \xi_{3}^{\prime} x y, \\
A_{00+}= & \left(-\alpha_{1}+\frac{1}{2} \alpha_{3}\right)+\left(\beta_{1}-\frac{1}{2} \beta_{3}-\sqrt{3} \gamma_{3}\right) y \\
& -\left(\zeta_{1}+\zeta_{3}\right)\left(y^{2}+\frac{1}{3} x^{2}\right) \\
& -\left(\xi_{1}+\xi_{3}+\xi_{3}^{\prime}\right)\left(y^{2}-\frac{1}{3} x^{2}\right) \\
& +\left(\xi_{1}+\xi_{3}-\xi_{3}^{\prime}\right)\left(y^{2}-\frac{1}{3} x^{2}\right) . \\
& -\left(2 \zeta_{1}+2 \zeta_{3}\right)\left(y^{2}+\frac{1}{3} x^{2}\right) \\
A_{++-}= & \left.-2 \alpha_{1}+\alpha_{3}\right)+\left(-\beta_{1}+\frac{1}{2} \beta_{3}-\sqrt{3} \gamma_{3}\right) y \\
&
\end{aligned}
$$

To NLO in the chiral expansion, the $K \rightarrow 3 \pi$ amplitudes were first calculated by Kambor et al. (1991) in the 
isospin limit. Their analysis was repeated and updated by Bijnens et al. (2003), confirming the main observation of Kambor et al. (1992, 1991) that inclusion of NLO corrections significantly improves the LO current algebra amplitudes. One main reason for the much better agreement with experimental data is that the quadratic slope parameters $\zeta_{1}, \ldots, \xi_{3}^{\prime}$ vanish at LO. On the other hand, NLO CHPT only provides the leading contributions for these quadratic slope parameters, which moreover depend on a number of LECs. Some assumptions about the combinations of NLO LECs occurring in the amplitudes must be made for a comparison with experiment.

Experiments on $K \rightarrow 3 \pi$ decays provide information on the rates and on the Dalitz plot distributions. The latter are conventionally expanded up to second order in $x, y$ (assuming again $\mathrm{CP}$ conservation):

$$
\left|\frac{A\left(s_{1}, s_{2}, s_{3}\right)}{A\left(s_{0}, s_{0}, s_{0}\right)}\right|^{2}=1+g y+h y^{2}+k x^{2},
$$

except for $K_{S} \rightarrow \pi^{+} \pi^{-} \pi^{0}$ [see Eq. [5.33]]. The experimental data available at the end of 2004 were confronted with NLO CHPT by Bijnens and Borg (2004, 2005a b), including also isospin-violating and radiative corrections. Assuming that the additional electromagnetic LECs at NLO in the Lagrangian (2.18) all vanish at a certain scale, their conclusion was that isospin breaking in $K \rightarrow 3 \pi$ is in general small. That analysis seems worth repeating, not only because of better knowledge of LECs reviewed in Sec. III, but also because of the more precise experimental information on the Dalitz plot slopes in Eq. (5.34), in particular for the $K^{ \pm}$modes from NA48/2 [see Batley et al. (2010a) and references therein].

\section{CP violation in $K \rightarrow 3 \pi$ decays}

The decay $K_{S} \rightarrow 3 \pi^{0}$ violates CP. In analogy to $K^{0} \rightarrow$ $2 \pi$ in Eq. (5.13), one defines the amplitude ratio

$$
\eta_{000}=\left.\frac{A_{000}^{S}}{A_{000}^{L}}\right|_{x=y=0}=\epsilon+\epsilon_{000}^{\prime} .
$$

The parameter $\epsilon_{000}^{\prime}$ is a measure of direct CP violation. To lowest order in CHPT, there is a simple relation between $\epsilon_{000}^{\prime}$ and $\epsilon^{\prime}$ (D'Ambrosio and Isidori, 1998; Li and Wolfenstein, 1980, which implies

$$
\left|\epsilon_{000}^{\prime}\right| \ll|\epsilon| .
$$

Therefore, it will be very difficult to detect direct $\mathrm{CP}$ violation in this decay. In fact, $K_{S} \rightarrow 3 \pi^{0}$ has not been observed at all so far. The accurate theoretical prediction

$$
\operatorname{BR}\left(K_{S} \rightarrow 3 \pi^{0}\right)=1.9 \times 10^{-9}
$$

should be compared with the best upper bound (Ambrosino et al. 2005)

$$
\operatorname{BR}\left(K_{S} \rightarrow 3 \pi^{0}\right)<1.2 \times 10^{-7} \quad(90 \% \text { C.L. }),
$$

\begin{tabular}{|c|c|c|c|}
\hline$A_{g}^{C}$ & $A_{g}^{N}$ & Ref. & \\
\hline$(-2.4 \pm 1.2) \times 10^{-5}$ & $(1.1 \pm 0.7) \times 10^{-5}$ & Gámiz et al. & (2003) \\
\hline$(-1.5 \pm 2.2) \times 10^{-4}$ & $(1.8 \pm 1.8) \times 10^{-4}$ & Batley et al. & $(2007)$ \\
\hline
\end{tabular}

TABLE V Dalitz slope asymmetries for $K^{ \pm} \rightarrow 3 \pi$ decays: comparison between theory (Gámiz et al. 2003 ) and experiment (Batley et al. 2007).

corresponding to

$$
\left|\eta_{000}\right|<0.018 .
$$

In the search for direct $\mathrm{CP}$ violation in $K$ decays, the three-pion decays of charged kaons have played a prominent role. In addition to rate asymmetries, both theory and experiment have paid special attention to asymmetries in the linear Dalitz plot parameter $g$ defined in Eq. (5.34).

After a number of conflicting estimates [reviewed by D'Ambrosio and Isidori (1998)], the theoretical state-ofthe-art Dalitz plot asymmetries come from a NLO CHPT calculation of Gámiz et al. (2003) [see also Prades (2008); Prades et al. (2007)]. In fact, this calculation also involves an estimate of the dominant contributions to the absorptive parts of the relevant amplitudes of $\mathcal{O}\left(G_{8} p^{6}\right)$.

The linear Dalitz plot slopes $g_{C, N}^{ \pm}$refer to the decays of $K^{ \pm}$into three charged pions $(C)$, and one charged and two neutral pions $(N)$, respectively. The CP-violating quantities of interest are the slope asymmetries

$$
A_{g}^{C, N}=\frac{g_{C, N}^{+}-g_{C, N}^{-}}{g_{C, N}^{+}+g_{C, N}^{-}} .
$$

These asymmetries depend at $\mathrm{LO}$ on the imaginary parts of the LECs $G_{8}$ and $g_{\text {ewk }}$ [Eqs. 2.16 and 2.18] only. Gámiz et al. (2003) found that $A_{g}^{C}$ is relatively insensitive to NLO LECs whereas $A_{g}^{N}$ is less stable. The theoretical results are compared with the most precise experimental data in Table $\mathrm{V}$.

To a good approximation, $\epsilon^{\prime} / \epsilon$ also depends on the imaginary parts of the LO LECs $G_{8}$ and $g_{\text {ewk }}$ only (see Sec. V.A.2). Therefore, one can establish bounds for $A_{a}^{C}$ within the SM, using the experimental value for $\epsilon^{\prime} / \epsilon$ (Prades, 2008).

\section{2. $\pi \pi$ scattering lengths from $K \rightarrow 3 \pi$ decays near threshold}

Because of the $\pi^{+}-\pi^{0}$ mass difference there is a cusp in the $M_{\pi^{0} \pi^{0}}$ distribution at $M_{\pi^{0} \pi^{0}}=2 M_{\pi^{+}}$in $K \rightarrow 3 \pi$ decays with two $\pi^{0}$ in the final state. It was first seen in $K^{ \pm} \rightarrow \pi^{ \pm} \pi^{0} \pi^{0}$ (Batley et al. 2006, 2009a), more recently also in $K_{L} \rightarrow 3 \pi^{0}$ (Abouzaid et al., 2008a). It is due to the charge exchange scattering of pions in the final state (Budini and Fonda, 1961, Cabibbo, 2004)

$$
K^{ \pm} \rightarrow \pi^{ \pm}\left(\pi^{+} \pi^{-}\right)^{*} \rightarrow \pi^{ \pm} \pi^{0} \pi^{0} .
$$




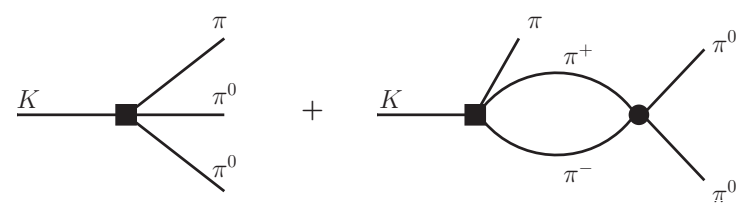

FIG. 7 Interference between tree and one-loop amplitudes generates a cusp at $M_{\pi^{0} \pi^{0}}^{2}=4 M_{\pi^{+}}^{2}$ in $K \rightarrow 3 \pi$ decays.

The interference between tree and one-loop amplitudes in Fig. 7 generates the cusp via the square-root singularity at $M_{\pi^{0} \pi^{0}}^{2}=4 M_{\pi^{+}}^{2}$. From Eq. (5.41) the effect is seen to be mainly sensitive to the combination of $\pi \pi$ scattering lengths

$$
a_{0}-a_{2} \sim A\left(\pi^{+} \pi^{-} \rightarrow \pi^{0} \pi^{0}\right) \text { thresh. }
$$

Various approaches have been pursued to extract the $\pi \pi$ scattering lengths from $K \rightarrow 3 \pi$ near threshold.

i. Following the original approach of Cabibbo (2004), based on unitarity and analyticity, a systematic expansion of the singular terms of the $M_{\pi^{0} \pi^{0}}$ distribution in powers of the scattering lengths was performed by Cabibbo and Isidori (2005).

ii. In a related method, unitarity and analyticity were combined with CHPT (Gámiz et al. 2007).

iii. A two-loop dispersive representation of $K \rightarrow 3 \pi$ amplitudes in the presence of isospin breaking is under construction (Kampf et al. 2009).

iv. In the most advanced approach, based on a nonrelativistic effective field theory (NRQFT), the $K \rightarrow$ $3 \pi$ amplitudes are expanded in powers of the scattering lengths and of the pion momenta in the $K$ rest frame (Bissegger et al., 2008; Colangelo et al. 2006; Gasser et al., 2011).

In the NRQFT approach of the Bern-Bonn group, the power counting involves a small parameter $\varepsilon$ that characterizes the size of pion three-momenta: $|\vec{p}| / M_{\pi}=\mathcal{O}(\varepsilon)$. In contrast to standard CHPT, the scattering lengths are not predicted but extracted from the data. The decay amplitudes are then given by a two-fold expansion in $\varepsilon$ and in the scattering lengths (denoted below generically as $a$ ). At any given order in $a$ and $\varepsilon$, only a finite number of graphs contribute because each loop is suppressed by one order in $\varepsilon$.

NRQFT is manifestly Lorentz invariant and therefore frame independent. Analyticity and unitarity are guaranteed as in standard CHPT. However, unlike in standard CHPT, the amplitudes are valid to all orders in the quark masses. They have been calculated up to $\mathcal{O}\left(\varepsilon^{4}, a \varepsilon^{5}, a^{2} \varepsilon^{2}\right)$ (Bissegger et al. 2008, Colangelo et al. 2006).

A major advantage of the NRQFT approach is that photons can be incorporated in a straightforward manner, allowing for the systematic inclusion of electromagnetic corrections (Bissegger et al. 2009). In the Coulomb

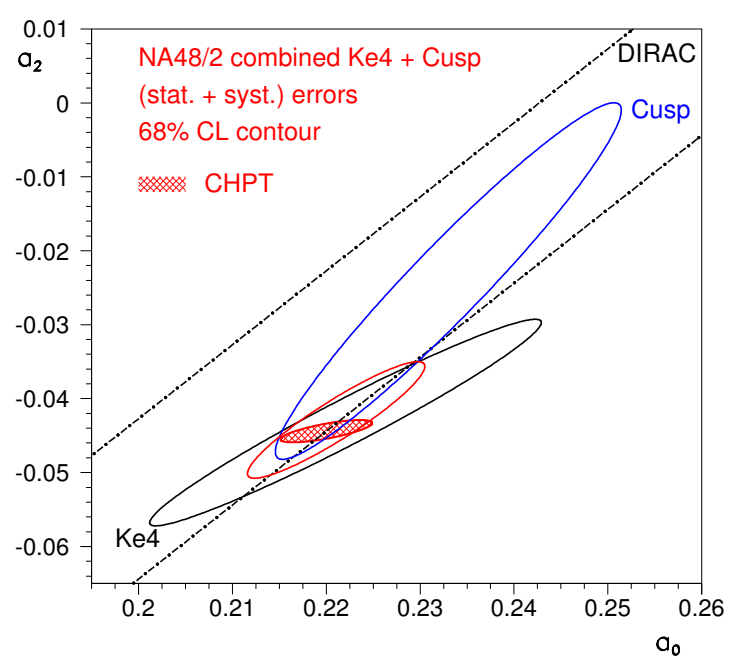

FIG. 8 NA48/2 $K_{e 4}$ and cusp results from two-parameter fits in the $\left(a_{0}, a_{2}\right)$ plane. The smallest contour corresponds to the combination of NA48/2 results. The cross-hatched ellipse is the CHPT prediction 4.92 of Colangelo et al. (2001a b). The dash-dotted lines correspond to the recent result from DIRAC (Adeva et al., 2011 ). We thank Brigitte Bloch-Devaux for updating the original figure from Batley et al. (2010c).

gauge, only transverse photons appear as internal lines in diagrams whereas the Coulomb photons give rise to a non-local vertex via the equations of motion. Of course, also real photon emission must be included, in particular to cancel infrared divergences. However, the nonrelativistic power counting then shows that finite bremsstrahlung effects are small near threshold.

The production of pionium, bound states of charged pions, upsets the nonrelativistic counting. A certain region around the cusp is therefore excluded from the data analysis such that one-photon exchange of $\mathcal{O}\left(a e^{2}\right)$ is sufficient.

The decay spectra were calculated to $\mathcal{O}\left(e^{2} \varepsilon^{4}\right)$ for all $K \rightarrow 3 \pi$ channels and, in addition, to $\mathcal{O}\left(e^{2} a \varepsilon^{2}\right)$ for the channels of main interest, $K^{+} \rightarrow \pi^{+} \pi^{0} \pi^{0}$ and $K_{L} \rightarrow 3 \pi^{0}$ (Bissegger et al. 2009). The radiatively corrected amplitudes were used in the analysis of NA48/2 data by Batley et al. (2009a) to extract the scattering lengths. Fig. 8 shows a comparison of the cusp analysis of Batley et al. (2009a) and the $K_{e 4}$ studies of Batley et al. (2010c). The smallest contour corresponds to the combination of the two methods. The result of the DIRAC experiment (Adeva et al., 2011) and the CHPT prediction of Colangelo et al. (2001a b) are shown as well. The corresponding numerical results are displayed in Table VI. The agreement between theory and experiment is impressive. 
TABLE VI Experimental and theoretical results for s-wave $\pi \pi$ scattering lengths. First line: cusp analysis of NA48/2 (Batley et al. 2009a) using the Bern-Bonn framework (Bissegger et al., 2008, Colangelo et al., 2006). The external error is due to the uncertainty in the ratio of the amplitudes for $K^{+} \rightarrow \pi^{+} \pi^{+} \pi^{-}$and $K^{+} \rightarrow \pi^{+} \pi^{0} \pi^{0}$. Second line: $K_{e 4}$ analysis of NA48/2 (Batley et al. 2010c). Third line: analysis of Colangelo et al. (2001a) on the basis of Roy equations and CHPT.

\begin{tabular}{|c|c|c|c|}
\hline & $a_{0}-a_{2}$ & $a_{0}$ & $a_{2}$ \\
\hline Batley et al. (2009a) & $0.2571(48)_{\text {stat }}(25)_{\text {syst }}(14)_{\text {ext }}$ & & $-0.0241(129)_{\text {stat }}(94)_{\text {syst }}(18)_{\text {ext }}$ \\
\hline Batley et al. (2010c) & & $0.2220(128)_{\text {stat }}(50)_{\text {syst }}(37)_{\text {th }}$ & $-0.0432(86)_{\text {stat }}(34)_{\text {syst }}(28)_{\text {th }}$ \\
\hline Colangelo et al. (2001a) & $0.264 \pm 0.004$ & $0.220 \pm 0.005$ & $-0.0444 \pm 0.0010$ \\
\hline
\end{tabular}
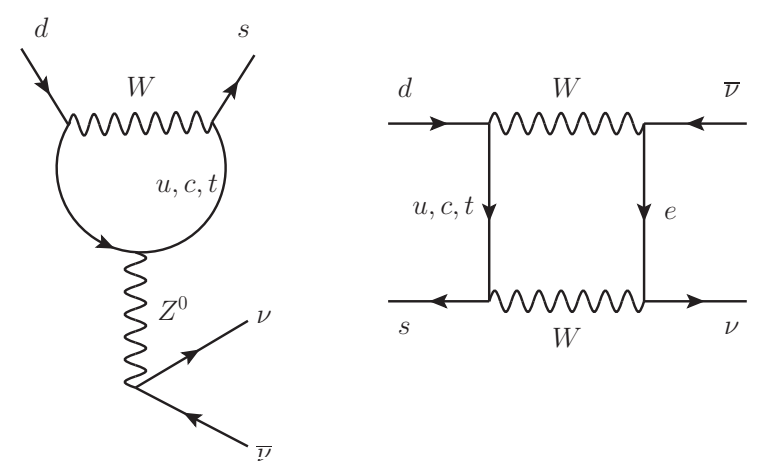

FIG. 9 Z-penguin and box contributions to $K \rightarrow \pi \nu \bar{\nu}$.

\section{RARE AND RADIATIVE DECAYS}

Kaon decays mediated by FCNC fall in the set of rare and radiative decays. These modes are suppressed in the SM and their main interest, other than their own understanding, relies on the possible observation of New Physics effects. Most of these processes are dominated by long-distance contributions, such as $K \rightarrow \gamma \gamma, K \rightarrow \gamma \gamma^{*}$, $K \rightarrow \pi \gamma^{*}$ and others. However, there are also processes governed by short-distance amplitudes, such as $K \rightarrow \pi \nu \bar{\nu}$.

Long-distance dominated decays have been studied in the chiral framework. The leading contributions, mostly $\mathcal{O}\left(p^{4}\right)$, have been evaluated within CHPT. In many of the processes estimates of the dominant NLO corrections have also been carried out.

\section{A. $K \rightarrow \pi \nu \bar{\nu}, \pi \pi \nu \bar{\nu}$}

The rare decays $K_{L} \rightarrow \pi^{0} \nu \bar{\nu}$ and $K^{ \pm} \rightarrow \pi^{ \pm} \nu \bar{\nu}$ can be predicted with a precision surpassing any other FCNC process involving quarks, thus making them a clean place to look for non-standard signals. The analysis of these processes makes use of the full arsenal of effective field theory, from short-distance effective Hamiltonians to CHPT. We outline below the main steps of the anal- ysis and then quote the final results for the branching ratios, with a brief discussion of the uncertainties.

Let us begin with a qualitative discussion that highlights the main features of these modes. In the SM, the $K \rightarrow \pi \nu \bar{\nu}$ decays proceed through FCNC amplitudes generated at the quark level by Z-penguin and box diagrams (see Fig. 9). Separating the contributions according to the intermediate up-type quark running inside the loops, the quark-level amplitude has the structure

$$
\begin{aligned}
A(s \rightarrow & d \nu \bar{\nu}) \sim \sum_{q=u, c, t} \lambda_{q} X_{\mathrm{SM}}\left(x_{q}\right) \\
& \sim \frac{m_{t}^{2}}{M_{W}^{2}} \lambda_{t}+\frac{m_{c}^{2}}{M_{W}^{2}} \ln \frac{M_{W}}{m_{c}} \lambda_{c}+\frac{\Lambda_{\mathrm{QCD}}^{2}}{M_{W}^{2}} \lambda_{u}
\end{aligned}
$$

where $\lambda_{q}=V_{q d} V_{q s}^{*}$ and $x_{q}=m_{q}^{2} / M_{W}^{2}$. The equation above reflects the quadratic ("hard") nature of the GIM mechanism. This in turn implies that the topquark contribution, which carries a large CP-violating phase, accounts for $\sim 68 \%$ of $A(s \rightarrow d \nu \bar{\nu})$ while the charm- and up-quark contributions amount to $\sim 29 \%$ and $\sim 3 \%$, respectively. So we see that the power-like GIM mechanism implies a large suppression of the $u$ quark (long-distance) contribution and complete dominance of the $\mathrm{CP}$-violating contribution by the $t$-quark term. These properties are often summarized by stating that the $K \rightarrow \pi \nu \bar{\nu}$ decays are "short-distance" dominated. The short-distance domination also implies that to a good approximation the interaction is described at low energy by one single local operator, $Q_{13}$ in Eq. 2.9, whose hadronic matrix element can be related to the form factors appearing in $K_{\ell 3}$ decays. All these features make it possible to predict $K \rightarrow \pi \nu \bar{\nu}$ rates very accurately.

The above qualitative discussion can be put on solid footing by systematically employing effective-theory techniques. As usual in the application to weak decays, three steps are required. (i) Determine the effective Lagrangian $\mathcal{L}_{\text {eff }}$ at the weak scale $\mu \sim M_{W}$ by integrating out the heavy gauge bosons and the top quark. In this case, the diagrams of Fig. 9 generate the operator $Q_{13}$. The Wilson coefficient $C_{13}$ is determined entirely in terms of $\lambda_{t}$ and $x_{t}$, and it now includes NLO QCD effects (Buchalla and Buras, 1999, Misiak and Urban, 1999) and two-loop electroweak corrections (Brod et al., 2011; 
Buchalla and Buras, 1998). (ii) Evolve $\mathcal{L}_{\text {eff }}$ down to a low hadronic scale using renormalization group techniques. In this step charm-loop contributions (the $c$ quark is still an active degree of freedom) generate corrections to $C_{13}$ proportional to $\lambda_{c} x_{c} \ln x_{c}$. These are known up to and including NNLO QCD effects (Buchalla and Buras, 1994; Buras et al., 2005, 2006) and NLO electroweak corrections (Brod and Gorbahn, 2008). At the charm threshold $\mu \sim m_{c}$ one integrates out the charm quark. At the current level of precision, one needs to keep not only the leading dimension-six operator, but also dimension-eight operators (Falk et al. 2001), whose relative importance is suppressed by $M_{K}^{2} / m_{c}^{2} \sim 15 \%$. (iii) Calculate hadronic matrix elements of the operators appearing in $\mathcal{L}_{\text {eff }}$ at a low scale $\mu \sim 1 \mathrm{GeV} . \mathcal{L}_{\text {eff }}$ contains the purely semileptonic operator $Q_{13}$, the dimension-eight operators generated at the charm threshold, and the $|\Delta S|=1$ fourquark operators, which generate long-distance contributions to $K \rightarrow \pi \nu \bar{\nu}$ (they correspond to the $u$-quark contributions to Z-penguin and box diagrams). The matrix element of $Q_{13}$ can be related to $K_{\ell 3}$ form factors (Marciano and Parsa, 1996). This analysis was recently updated by Mescia and Smith (2007) to include first-order isospin-breaking effects to NLO in CHPT as well as longdistance radiative corrections for the $K^{ \pm}$decay. Concerning the long-distance contributions and dimensioneight operators, the relevant matrix elements were calculated in CHPT (Isidori et al., 2005; Lu and Wise, 1994).

Owing to the CP properties of the $K_{2}^{0}$ state, the operator $Q_{13}$ only contributes to $K_{L} \rightarrow \pi^{0} \nu \bar{\nu}$ through a violation of the CP symmetry. The transition amplitude is completely dominated by direct $\mathrm{CP}$ violation, the contribution from $K^{0}-\overline{K^{0}}$ mixing being only of the order of $1 \%$. There exist tiny CP-conserving contributions through higher-order short-distance operators and longdistance corrections, but their effect on the branching ratio is negligible (Buchalla and Isidori, 1998).

Putting all the ingredients together, the predicted SM rates for $K \rightarrow \pi \nu \bar{\nu}$ decays can be written as

$$
\begin{aligned}
\operatorname{BR}\left(K_{L} \rightarrow \pi^{0} \nu \bar{\nu}\right) & =\kappa_{L}\left(\frac{\operatorname{Im} \lambda_{t}}{\lambda^{5}} X\right)^{2}\left(1-\delta_{\epsilon}\right), \\
\operatorname{BR}\left(K^{+} \rightarrow \pi^{+} \nu \bar{\nu}\right) & =\kappa_{+}\left(1+\Delta_{\mathrm{EM}}\right)\left[\left(\frac{\operatorname{Im} \lambda_{t}}{\lambda^{5}} X\right)^{2}\right. \\
+\left(\frac{\operatorname{Re} \lambda_{t}}{\lambda^{5}} X\right. & \left.\left.+\frac{\operatorname{Re} \lambda_{c}}{\lambda}\left(P_{c}+\delta P_{c, u}\right)\right)^{2}\right]
\end{aligned}
$$

where $\delta_{\epsilon}=\sqrt{2}|\epsilon|\left[1+P_{c} /\left(A^{2} \lambda\right)-\rho\right] / \eta$ contains the small $K^{0}-\overline{K^{0}}$ mixing contribution (Buchalla and Buras, 1996), with $\lambda=V_{u s}, A, \rho$ and $\eta$ the Wolfenstein CKM parameters. The overall factors $\kappa_{L,+}$ encode the hadronic matrix element related to $K_{\ell 3}$ data (Mescia and Smith, 2007):

$$
\begin{aligned}
& \kappa_{L}=(2.231 \pm 0.013) \times 10^{-10}(\lambda / 0.225)^{8}, \\
& \kappa_{+}=(5.173 \pm 0.025) \times 10^{-11}(\lambda / 0.225)^{8} .
\end{aligned}
$$

They are now dominated by experimental uncertainties in $K_{\ell 3}$. Defining the infrared-safe photon-inclusive rate by the cut $E_{\gamma}^{\mathrm{cms}}<20 \mathrm{MeV}$, the electromagnetic correction takes the value $\Delta_{\mathrm{EM}}=-0.003$ (Mescia and Smith, 2007). The top-quark contribution is given by $X=1.469 \pm 0.017$ (Brod et al., 2011; Buras et al., 2005, 2006), with an uncertainty due to the parametric error in $m_{t}$, matching scale $\mu_{t}$, and higher-order electroweak effects. The dimension-six charm contribution has the value $P_{c}=0.38 \pm 0.04$, with error dominated by the parametric uncertainty in $m_{c}$. Finally, the long-distance ( $u$ quark) and dimension-eight charm contributions can be lumped in $\delta P_{c, u}=0.04 \pm 0.02$ (Isidori et al., 2005). Taking the CKM matrix elements from global fits, one arrives at (Brod et al., 2011, Buras et al., 2005, 2006):

$$
\begin{aligned}
\operatorname{BR}\left(K_{L} \rightarrow \pi^{0} \nu \bar{\nu}\right) & =(2.4 \pm 0.4) \times 10^{-11}, \\
\operatorname{BR}\left(K^{+} \rightarrow \pi^{+} \nu \bar{\nu}\right) & =(0.78 \pm 0.08) \times 10^{-10} .
\end{aligned}
$$

In both cases the uncertainty is largely parametrical ( $\sim 80 \%$ for the $K_{L}$ mode and $\sim 70 \%$ for the $K^{+}$mode), due to CKM input, $m_{c}, m_{t}$ and $\alpha_{s}\left(M_{Z}\right)$. As the determination of CKM parameters improves in the next few years, we can expect to reach accuracies better than $10 \%$. In summary, from a theoretical perspective $K \rightarrow \pi \nu \bar{\nu}$ decays offer the cleanest window on non-standard contributions to the $s \rightarrow d$ transitions (complementary to B meson studies). Moreover, within the general context of flavor physics, $K \rightarrow \pi \nu \bar{\nu}$ decays are perhaps the most promising place to look for non-standard signals, due to their SM-specific suppression induced by CKM factors $\left[A(s \rightarrow d \nu \bar{\nu}) \propto V_{u s}^{5}\right]$. Even if one considers SM extensions with the same CKM suppression factor, $K \rightarrow \pi \nu \bar{\nu}$ decays still provide an excellent probe, because the SM contributions can be predicted very accurately.

On the experimental side, the charged kaon mode was observed (Artamonov et al. 2008), while only an upper bound on the neutral mode has been achieved (Ahn et al. 2008, 2010):

$$
\begin{aligned}
\operatorname{BR}\left(K^{+} \rightarrow \pi^{+} \nu \bar{\nu}\right) & =\left(1.73_{-1.05}^{+1.15}\right) \times 10^{-10} \\
\operatorname{BR}\left(K_{L} \rightarrow \pi^{0} \nu \bar{\nu}\right) & <2.6 \times 10^{-8} \quad(90 \% \text { C.L. }) .
\end{aligned}
$$

New experiments are under development at CERN (Spadaro, 2011) and J-PARC (Watanabe, 2010) for charged and neutral modes, respectively. These experiments aim to reach $\mathcal{O}(100)$ events (assuming SM rates), thus beginning to seriously probe the new-physics potential of these rare $K$ decays. Increased sensitivities could be obtained through the recent P996 proposal for a $K^{+} \rightarrow \pi^{+} \nu \bar{\nu}$ experiment at Fermilab and the higher kaon fluxes available at Project-X (Tschirhart, 2011).

Finally, let us mention that the decays $K_{L, S} \rightarrow \pi \pi \nu \bar{\nu}$ and $K^{ \pm} \rightarrow \pi^{ \pm} \pi^{0} \nu \bar{\nu}$ share the same feature of shortdistance domination as the corresponding single-pion modes, and could therefore provide another probe of the underlying $s \rightarrow d \nu \bar{\nu}$ transition within and beyond the SM. The calculation of the decay amplitudes requires taking the matrix element of the current $\bar{s} \gamma_{\mu}\left(1-\gamma_{5}\right) d$ between the kaon and two-pion states, which can be extracted from the measured $K_{\ell 4}$ decays using isospin sym- 
metry (Chiang and Gilman, 2000 Littenberg and Valencia, 1996), or directly calculated in CHPT (Geng et al., 1994). Theoretical predictions can be summarized as follows (Littenberg and Valencia, 1996):

$$
\begin{aligned}
& \operatorname{BR}\left(K_{L} \rightarrow\right.\left.\pi^{+} \pi^{-} \nu \bar{\nu}\right) \simeq \\
& 1.8 \times\left[(1.37-\rho)^{2}+0.17 \eta^{2}\right] \times 10^{-13} \\
& \operatorname{BR}\left(K_{L} \rightarrow\right.\left.\pi^{0} \pi^{0} \nu \bar{\nu}\right) \simeq(1.37-\rho)^{2} \times 10^{-13} \\
& \operatorname{BR}\left(K^{ \pm} \rightarrow \pi^{ \pm} \pi^{0} \nu \bar{\nu}\right) \simeq 7 \times\left[(1.37-\rho)^{2}+\eta^{2}\right] \times 10^{-15} .
\end{aligned}
$$

Experimental searches are still far above the expected SM rates and have reached the following $90 \%$ C.L. limits:

$$
\begin{aligned}
\operatorname{BR}\left(K^{+} \rightarrow \pi^{+} \pi^{0} \nu \bar{\nu}\right) & <4.3 \times 10^{-5} \text { Adler et al. 2001), } \\
\operatorname{BR}\left(K_{L} \rightarrow \pi^{0} \pi^{0} \nu \bar{\nu}\right) & <8.1 \times 10^{-7} \text { (Ogata et al. 2011). }
\end{aligned}
$$

\section{B. $K \rightarrow \gamma^{(*)} \gamma^{(*)}$}

The amplitude for a transition of the type

$$
K(p) \longrightarrow \gamma^{*}\left(q_{1}\right) \gamma^{*}\left(q_{2}\right)
$$

is determined by a tensor amplitude $M^{\mu \nu}\left(q_{1}, q_{2}\right)$ that has the most general form compatible with gauge invariance:

$$
\begin{aligned}
M^{\mu \nu}= & {\left[g^{\mu \nu}-\frac{q_{1}^{\mu} q_{1}^{\nu}}{q_{1}^{2}}-\frac{q_{2}^{\mu} q_{2}^{\nu}}{q_{2}^{2}}+\frac{q_{1} \cdot q_{2}}{q_{1}^{2} q_{2}^{2}} q_{1}^{\mu} q_{2}^{\nu}\right] M_{K}^{2} a\left(q_{1}^{2}, q_{2}^{2}\right) } \\
+ & {\left[q_{2}^{\mu} q_{1}^{\nu}-q_{1} \cdot q_{2}\left(\frac{q_{1}^{\mu} q_{1}^{\nu}}{q_{1}^{2}}+\frac{q_{2}^{\mu} q_{2}^{\nu}}{q_{2}^{2}}-\frac{q_{1} \cdot q_{2}}{q_{1}^{2} q_{2}^{2}} q_{1}^{\mu} q_{2}^{\nu}\right)\right] } \\
& \times b\left(q_{1}^{2}, q_{2}^{2}\right) \\
+ & i \varepsilon^{\mu \nu \rho \sigma} q_{1 \rho} q_{2 \sigma} c\left(q_{1}^{2}, q_{2}^{2}\right) .
\end{aligned}
$$

Thus, for instance, the amplitude into two real photons is given by $A=M^{\mu \nu}\left(q_{1}, q_{2}\right) \varepsilon_{\mu}\left(q_{1}\right) \varepsilon_{\nu}\left(q_{2}\right)$. Bose symmetry implies that the invariant amplitudes $a\left(q_{1}^{2}, q_{2}^{2}\right), b\left(q_{1}^{2}, q_{2}^{2}\right)$ and $c\left(q_{1}^{2}, q_{2}^{2}\right)$ are symmetric functions of their arguments. If $\mathrm{CP}$ is conserved the amplitudes $a$ and $b$ contribute to $K_{1}^{0}\left(K_{S}\right) \rightarrow \gamma^{*} \gamma^{*}$ while the amplitude $c$ determines $K_{2}^{0}\left(K_{L}\right) \rightarrow \gamma^{*} \gamma^{*}$. When one of the photons is on-shell ( $q_{1}^{2}=0$ for instance), $M^{\mu \nu}$ is described by two invariant amplitudes,

$$
\begin{aligned}
M^{\mu \nu}= & \left(q_{2}^{\mu} q_{1}^{\nu}-q_{1} \cdot q_{2} g^{\mu \nu}\right) b\left(0, q_{2}^{2}\right) \\
& +i \varepsilon^{\mu \nu \rho \sigma} q_{1 \rho} q_{2 \sigma} c\left(0, q_{2}^{2}\right),
\end{aligned}
$$

which also remains valid for both photons on-shell.

These processes are dominated by long-distance dynamics, in particular those with both photons on-shell or when off-shell photons produce lepton pairs. Shortdistance amplitudes give only tiny contributions.

Since the photon does not couple directly to neutral particles, the only possible local contributions should come from field strength tensors, which are absent at $\mathcal{O}\left(p^{2}\right)$. Moreover, the $\mathcal{O}\left(p^{4}\right)$ Lagrangians $\mathcal{L}_{G_{8} p^{4}}^{\Delta S=1}$ and $\mathcal{L}_{G_{27} p^{4}}^{\Delta S=1}$ in Eq. 2.16 only contain two operators with $F^{\mu \nu}$
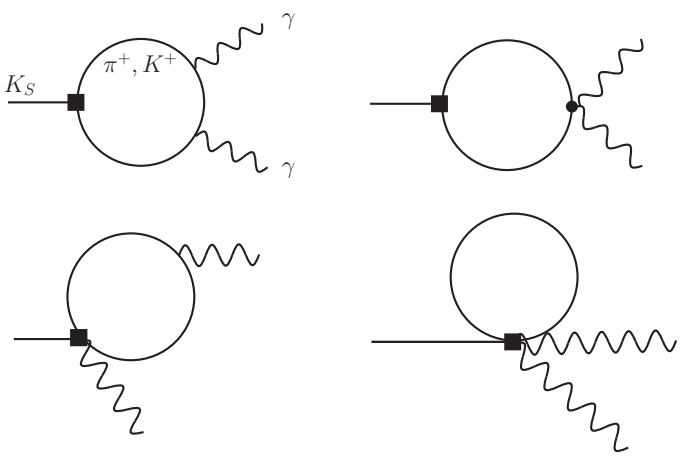

FIG. 10 Lowest-order $\left[\mathcal{O}\left(p^{4}\right)\right]$ contributions to $K_{S} \rightarrow \gamma \gamma$.

terms, but they are coupled to at least two charged particles. Therefore, the leading $K \rightarrow \gamma^{*} \gamma^{*}$ contribution is generated by a non-local $\mathcal{O}\left(p^{4}\right)$ loop amplitude that is necessarily finite. In the following and unless explicitly stated we assume CP invariance.

\section{1. $K_{S} \rightarrow \gamma \gamma$}

The photons produced in this decay have parallel polarizations $\left(F_{\mu \nu} F^{\mu \nu}\right)$ and, up to one loop, there is no short-distance contribution due to Furry's theorem (Gaillard and Lee, 1974b). This decay arises, at leading order, from a finite two-pion one-loop amplitude with one vertex from $\mathcal{L}_{G_{8} p^{2}}^{\Delta S=1}$ or $\mathcal{L}_{G_{27} p^{2}}^{\Delta S=1}$ (D'Ambrosio and Espriu 1986 Goity, 1987), as shown in Fig. 10. Its contribution is

$$
b^{(4)}(0,0)=\frac{2}{\pi} \alpha F_{0}\left(G_{8}+\frac{2}{3} G_{27}\right)\left(1-r_{\pi}^{2}\right) F\left(\frac{1}{r_{\pi}^{2}}\right),
$$

where $r_{\mathrm{P}}=M_{P} / M_{K}$ and $F(x)$ is given in Eq. (A1). The charged-kaon loop contribution vanishes because of the factor $\left(1-r_{K}^{2}\right)=0$.

The decay width is given by

$$
\Gamma\left(K_{S} \rightarrow \gamma \gamma\right)=\frac{M_{K}^{3}}{64 \pi}|b(0,0)|^{2} .
$$

Taking the LO values of the couplings $G_{8}$ and $G_{27}$, Eq. 6.14 results in $\operatorname{BR}\left(K_{S} \rightarrow \gamma \gamma\right)=2.0 \times 10^{-6}$, which compares rather well with the present experimental world average (Nakamura et al. 2010)

$$
\operatorname{BR}\left(K_{S} \rightarrow \gamma \gamma\right)=(2.63 \pm 0.17) \times 10^{-6} .
$$

Although the full $\mathcal{O}\left(p^{6}\right)$ amplitude has not been calculated, the dominant effects were implemented through (i) the inclusion of unitarity corrections from $K_{S} \rightarrow \pi \pi \rightarrow$ $\pi^{+} \pi^{-} \rightarrow \gamma \gamma$ (Kambor and Holstein, 1994), and (ii) a local contribution introducing an unknown coupling constant (Buchalla et al. 2003),

$$
b^{(4+6)}(0,0)=\frac{2 \alpha F_{0}}{M_{K}^{2}} B\left(M_{K}^{2}\right)+\frac{4 \alpha G_{8}}{\pi F_{0}} M_{K}^{2}\left(1-r_{\pi}^{2}\right) a_{1},
$$




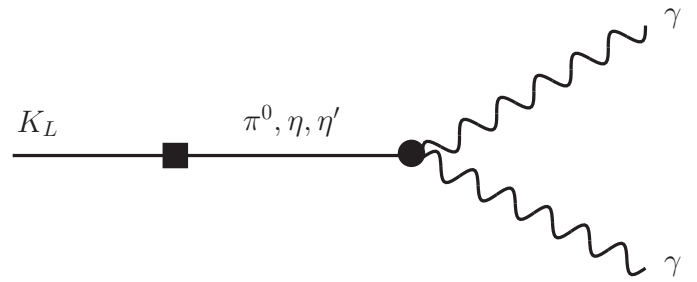

FIG. 11 Dominant contribution to $K_{L} \rightarrow \gamma \gamma$.

where $B(s)$ can be found in Kambor and Holstein (1994). Including the 27-plet contribution in $B(s)$, the experimental rate 6.16) implies $a_{1}=(-1.2 \pm 1.3) \times 10^{-3}$, showing that unitarity corrections are enough to reproduce the measured branching fraction.

\section{2. $K_{L} \rightarrow \gamma \gamma$}

This decay produces photons with perpendicular polarizations $\left(\varepsilon_{\mu \nu \rho \sigma} F^{\mu \nu} F^{\rho \sigma}\right)$ and then it is the amplitude $c(0,0)$ in Eq. 6.13 that contributes. Owing to its GIM suppression, the short-distance amplitude only gives a few-percent contribution to the full width (Gaillard and Lee, 1974b; Herrlich and Kalinowski, 1992, Ma and Pramudita, 1981; Pramudita, 1988). Accordingly this decay is also dominated by long-distance dynamics.

The dominant contribution, shown in Fig. 11, is given by the weak transition of the kaon into a non-flavored pseudoscalar meson and its corresponding decay into two photons (Ma and Pramudita, 1981), the latter being determined by the anomalous Lagrangian $\mathcal{L}_{\mathrm{WZW}}$ in Eq. (2.15). At lowest order in the chiral SU(3) expansion $\left[\mathcal{O}\left(p^{4}\right)\right]$, only $\pi^{0}$ and $\eta_{8}$ propagate. However, the amplitude vanishes exactly due to the Gell-Mann-Okubo mass relation (Gell-Mann, 1961, Okubo, 1962). Therefore, the decay starts at $\mathcal{O}\left(p^{6}\right)$ where the singlet $\eta_{1}$ state is also included:

$$
c^{(6)}(0,0)=-\frac{2}{\pi} \alpha F_{0}\left(G_{8}-G_{27}\right) F_{2}(\hat{\rho}, \xi, \theta),
$$

where

$$
\begin{aligned}
F_{2}=\frac{1}{1-r_{\pi}^{2}}+\frac{1}{3\left(1-r_{\eta}^{2}\right)}[(1+\xi) \cos \theta+2 \sqrt{2} \hat{\rho} \sin \theta] \\
\times\left[\frac{F_{\pi}}{F_{\eta_{8}}} \cos \theta-2 \sqrt{2} \frac{F_{\pi}}{F_{\eta_{0}}} \sin \theta\right] \\
-\frac{1}{3\left(1-r_{\eta^{\prime}}^{2}\right)}[2 \sqrt{2} \hat{\rho} \cos \theta-(1+\xi) \sin \theta] \\
\times\left[\frac{F_{\pi}}{F_{\eta_{8}}} \sin \theta+2 \sqrt{2} \frac{F_{\pi}}{F_{\eta_{0}}} \cos \theta\right] .
\end{aligned}
$$

Here $\theta$ is the mixing angle between $\eta_{8}$ and $\eta_{1}$ states:

$$
\left(\begin{array}{c}
\eta \\
\eta^{\prime}
\end{array}\right)=\left(\begin{array}{cc}
\cos \theta & -\sin \theta \\
\sin \theta & \cos \theta
\end{array}\right)\left(\begin{array}{l}
\eta_{8} \\
\eta_{1}
\end{array}\right) .
$$

Although there is still some discussion on the value of $\theta$, we take $\theta=-20^{\circ}$ arising in the large- $N_{C}$ analyses (Herrera-Siklódy et al. 1998 , Kaiser and Leutwyler, 1998). $\xi$ parametrizes the amount of SU(3) breaking (Donoghue et al., 1986):

$$
\xi=\sqrt{3} \frac{\left\langle\eta_{8}\left|\mathcal{L}^{|\Delta S|=1}\right| K_{2}^{0}\right\rangle}{\left\langle\pi^{0}\left|\mathcal{L}^{|\Delta S|=1}\right| K_{2}^{0}\right\rangle}-1,
$$

while $\hat{\rho}$ carries the information of the breaking of nonet symmetry through the weak interactions of the singlet at $\mathcal{O}\left(p^{2}\right)$ (Donoghue et al. 1984):

$$
\hat{\rho}=-\sqrt{\frac{3}{8}} \frac{\left\langle\eta_{1}\left|\mathcal{L}^{|\Delta S|=1}\right| K_{2}^{0}\right\rangle}{\left\langle\pi^{0}\left|\mathcal{L}^{|\Delta S|=1}\right| K_{2}^{0}\right\rangle}
$$

If $\mathrm{SU}(3)$ and nonet symmetries are exact we have $\xi=0$ and $\hat{\rho}=1$. Finally, $F_{\pi}, F_{\eta_{8}}$ and $F_{\eta_{1}}$ are the decay constants of the pion, $\eta_{8}$ and $\eta_{1}$, respectively. The values of the symmetry-breaking parameters are still not precisely known. We take $F_{\eta_{8}} / F_{\pi}=1.34$ and $F_{\eta_{1}} / F_{\pi}=1.0$ from Kaiser and Leutwyler (1998) [see also Feldmann and Kroll (2002)]. The SU(3) breaking parameter was estimated to be $\xi \simeq 0.17$ (Donoghue et al., 1986), but this value was challenged with the claim that it cancels with an additional $s \rightarrow d g g$ contribution (He et al. 2003). Hence we will consider, conservatively, $\xi \sim 0.0-0.2$. Finally, dominance of the pion pole seems to require a small breaking of nonet symmetry, $\hat{\rho} \simeq 0.8$ (Cheng, 1990, D'Ambrosio and Portolés, 1998a).

The decay width is given by Eq. 6.15) with the function $c(0,0)$ instead of $b(0,0)$. If we consider the experimental determination (Nakamura et al. 2010 )

$$
\operatorname{BR}\left(K_{L} \rightarrow \gamma \gamma\right)=(5.47 \pm 0.04) \times 10^{-4},
$$

a value of $\theta=-20^{\circ}$ accommodates $\xi=0$ with a value of $\hat{\rho} \simeq 0.7$ while $\xi=0.2$ requires $\hat{\rho} \simeq 0.8$.

\section{3. $K_{S} \rightarrow \gamma \ell^{+} \ell^{-}$}

The amplitude for processes with one off-shell photon decaying into a lepton pair is given by

$$
A=\frac{e}{q_{2}^{2}} M^{\mu \nu}\left(q_{1}, q_{2}\right) \varepsilon_{\mu}^{*}\left(q_{1}\right) \bar{u}(k) \gamma_{\nu} v\left(k^{\prime}\right),
$$

with $q_{2}=k+k^{\prime}$. At $\mathcal{O}\left(p^{4}\right)$ the amplitude $b\left(0, q_{2}^{2}\right)$ in Eq. (6.13) is uniquely determined by a one-loop calculation of the $K_{1}^{0} \rightarrow \gamma \gamma^{*}$ transition (Ecker et al., 1988, Sehgal, 1973). The dominant octet contribution is

$$
b^{(4)}\left(0, q_{2}^{2}\right)=\frac{4}{\pi} G_{8} \alpha F_{0}\left(1-r_{\pi}^{2}\right) H(z),
$$

where $z=q_{2}^{2} / M_{K}^{2}$ and $H(z)$ is given in Eq. A3. The result for the spectrum in $q_{2}^{2}$ is usually normalized to the two-photon width as

$$
\frac{1}{\Gamma_{\gamma \gamma}} \frac{d \Gamma}{d z}=\frac{2}{z}(1-z)^{3}\left|\frac{H(z)}{H(0)}\right|^{2} \frac{1}{\pi} \operatorname{Im} \Pi(z),
$$


with $\operatorname{Im} \Pi(z)$ the electromagnetic spectral function associated to the lepton pair:

$$
\frac{1}{\pi} \operatorname{Im} \Pi(z)=\frac{\alpha}{3 \pi}\left(1+2 \frac{r_{\ell}^{2}}{z}\right) \sqrt{1-4 \frac{r_{\ell}^{2}}{z}} \theta\left(z-4 r_{\ell}^{2}\right)
$$

where $r_{\ell}=m_{\ell} / M_{K} . \quad \Gamma_{\gamma \gamma} \equiv \Gamma\left(K_{S} \rightarrow \gamma \gamma\right)$ is given by $b^{(4)}(0,0)$ in Eq. 6.14 [notice that $H(0)=-\frac{1}{2} F\left(1 / r_{\pi}^{2}\right)$ ].

Integrating the spectrum, one predicts the ratios

$$
\frac{\Gamma\left(K_{S} \rightarrow \gamma \ell^{+} \ell^{-}\right)}{\Gamma\left(K_{S} \rightarrow \gamma \gamma\right)}= \begin{cases}1.2 \times 10^{-2} & (\ell=e) \\ 2.8 \times 10^{-4} & (\ell=\mu)\end{cases}
$$

These processes have not been measured yet.

\section{4. $K_{L} \rightarrow \gamma \ell^{+} \ell^{-}$}

As in the $K_{S}$ decay, the normalized spectrum is given by Eq. 6.26 with the ratio $H(z) / H(0)$ substituted by

$$
f(z) \equiv \frac{c\left(0, q_{2}^{2}\right)}{c(0,0)}=1+b z+\mathcal{O}\left(z^{2}\right) .
$$

Being of higher order in CHPT, our knowledge of the form factor $f(z)$ is unfortunately rather limited. The decay mechanism in Fig. 11 generates an unambiguous contribution from the electromagnetic form factor of the $\pi^{0}$ $\left(\eta, \eta^{\prime}\right)$, which amounts to a slope $b_{V}=M_{K}^{2} / M_{\rho}^{2} \simeq 0.41$. However, there are other possible contributions that have been analyzed only within explicit models (Bergström et al., 1983, 1990; D'Ambrosio and Portolés, 1997; Ecker et al., 1990, Sehgal, 1973), giving slopes in the range $b \simeq 0.8-1.0$.

The experimental data can be fitted with a simple parametrization such as (D'Ambrosio et al., 1998b)

$$
\left.f(z)\right|_{\mathrm{DIP}}=1+\alpha_{\mathrm{DIP}} \frac{z}{z-M_{\rho}^{2} / M_{K}^{2}},
$$

which provides the slope $b^{\text {DIP }}=-\alpha_{\text {DIP }} M_{K}^{2} / M_{\rho}^{2}$. A recent determination by the $\mathrm{KTeV}$ collaboration Abouzaid et al. 2007b) for the electron case finds $\alpha_{\mathrm{DIP}}=-1.73 \pm$ $0.05\left(b^{\mathrm{DIP}}=0.71 \pm 0.02\right)$, while $\alpha_{\mathrm{DIP}}=-1.54 \pm 0.10$ $\left(b_{\mathrm{DIP}}=0.63 \pm 0.04\right)$ was previously extracted from the muon mode (Alavi-Harati et al. 2001c).

\section{5. $K_{L} \rightarrow \ell_{1}^{+} \ell_{1}^{-} \ell_{2}^{+} \ell_{2}^{-}$}

The dominant long-distance amplitude for this process is driven by the creation of two lepton pairs:

$$
A=\frac{4 \pi \alpha}{q_{1}^{2} q_{2}^{2}} M^{\mu \nu}\left(q_{1}, q_{2}\right) \bar{u}\left(k_{1}\right) \gamma_{\mu} v\left(k_{1}^{\prime}\right) \bar{u}\left(k_{2}\right) \gamma_{\nu} v\left(k_{2}^{\prime}\right),
$$

with $q_{i}=k_{i}+k_{i}^{\prime}$. If $\mathrm{CP}$ is conserved only the amplitude $c\left(q_{1}, q_{2}\right)$ in Eq. 6.12 contributes. The invariant spectrum in the variables $z_{i}=q_{i}^{2} / M_{K}^{2}$, normalized to $\Gamma_{\gamma \gamma} \equiv \Gamma\left(K_{L} \rightarrow \gamma \gamma\right)$, is given by

$$
\begin{aligned}
\frac{1}{\Gamma_{\gamma \gamma}} \frac{d^{2} \Gamma}{d z_{1} d z_{2}}= & \frac{2}{z_{1} z_{2}}\left(\frac{\alpha}{3 \pi}\right)^{2}\left|f\left(z_{1}, z_{2}\right)\right|^{2} \lambda^{3 / 2}\left(1, z_{1}, z_{2}\right) \\
& \times \prod_{i=1,2}\left(1+2 \frac{r_{\ell_{i}}^{2}}{z_{i}}\right) \sqrt{1-4 \frac{r_{\ell_{i}}^{2}}{z_{i}}},
\end{aligned}
$$

where $f\left(z_{1}, z_{2}\right)=c\left(q_{1}^{2}, q_{2}^{2}\right) / c(0,0)$. A parametrization suitable to analyze the data is provided by (D'Ambrosio et al. 1998b)

$$
\begin{aligned}
\left.f\left(z_{1}, z_{2}\right)\right|_{\mathrm{DIP}}= & 1+\alpha_{\mathrm{DIP}} \sum_{i=1,2} \frac{z_{i}}{z_{i}-M_{\rho}^{2} / M_{K}^{2}} \\
& +\beta_{\mathrm{DIP}} \prod_{i=1,2} \frac{z_{i}}{z_{i}-M_{\rho}^{2} / M_{K}^{2}} .
\end{aligned}
$$

The channels $\ell_{1}=\ell_{2}=e$ and $\ell_{1}=e, \ell_{2}=\mu$ have already been observed experimentally. The first one is only sensitive to the parameter $\alpha_{\text {DIP }}$ due to the low invariant mass of the $e^{+} e^{-}$pairs (Alavi-Harati et al. $2001 \mathrm{~d}$ ). Although the second channel should be more suitable to extract the parameter $\beta_{\mathrm{DIP}}$, in practice that is still not feasible due to poor statistics Alavi-Harati et al. $2003 \mathrm{~b}$ ). Consequently, $\beta_{\mathrm{DIP}}=0$ is assumed and $\alpha_{\mathrm{DIP}}$ is extracted. The most precise determination is obtained for the channel with muons giving $\alpha_{\text {DIP }}=-1.59 \pm 0.37$, in good agreement with the results from $K_{L} \rightarrow \gamma \mu^{+} \mu^{-}$. The world average is dominated by the $K_{L} \rightarrow \gamma e^{+} e^{-}$ mode and is given by $\alpha_{\text {DIP }}=-1.69 \pm 0.08$. A complete lowest-order calculation of QED radiative corrections was also performed (Barker et al. , 2003). Its impact on the slope $\alpha_{\text {DIP }}$ could amount to a $15 \%$ correction, well within the present error.

The high- $q^{2}$ behaviour of the function $f\left(z_{1}, z_{2}\right)$ in the model of D'Ambrosio et al. (1998b) enforces a constraint on its parameters that is violated only in a very mild way, namely the sum rule $1+2 \alpha_{\text {DIP }}+\beta_{\text {DIP }}=0$. Hence, from the world average value of $\alpha_{\text {DIP }}$, we can estimate the phenomenologically elusive second parameter: $\beta_{\mathrm{DIP}} \simeq 2.4$.

\section{C. $K \rightarrow \ell^{+} \ell^{-}$} by

The most general amplitude for this process is given

$$
A\left(K \rightarrow \ell^{+} \ell^{-}\right)=\bar{u}(k)\left(i B+C \gamma_{5}\right) v\left(k^{\prime}\right) .
$$

If $\mathrm{CP}$ is conserved the amplitude $B$ (p-wave $\left.{ }^{3} P_{0}\right)$ determines $K_{1}^{0} \rightarrow \ell^{+} \ell^{-}$while $K_{2}^{0} \rightarrow \ell^{+} \ell^{-}$proceeds via the s-wave ${ }^{1} S_{0}$ amplitude $C$. The associated width is

$$
\Gamma\left(K \rightarrow \ell^{+} \ell^{-}\right)=\frac{M_{K}}{8 \pi} \beta_{\ell}\left(\beta_{\ell}^{2}|B|^{2}+|C|^{2}\right),
$$

with $\beta_{\ell}=\sqrt{1-4 m_{\ell}^{2} / M_{K}^{2}}$. 


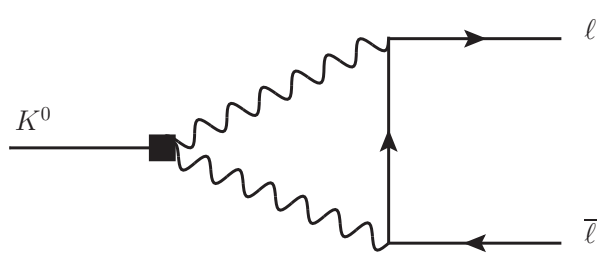

FIG. 12 Two-photon contribution to $K^{0} \rightarrow \ell^{+} \ell^{-}$.

\section{1. $K_{S} \rightarrow \ell^{+} \ell^{-}$}

The main contribution comes from the amplitude $B_{\gamma \gamma}$ that gives the transition $K_{S} \rightarrow \gamma^{*} \gamma^{*} \rightarrow \ell^{+} \ell^{-}$(Ecker and Pich, 1991). This two-loop amplitude is finite because chiral symmetry forbids any CP-invariant local contribution at this order. The result can be written as

$$
\begin{aligned}
R_{S}^{\ell} & =\frac{\Gamma\left(K_{S} \rightarrow \ell^{+} \ell^{-}\right)}{\Gamma\left(K_{S} \rightarrow \gamma \gamma\right)} \\
& =\frac{\alpha^{2} \beta_{\ell}^{2} m_{\ell}^{2}}{2 \pi^{2}|H(0)|^{2} M_{K}^{2}}\left|I_{\ell, \text { disp }}+i I_{\ell, \text { abs }}\right|^{2},
\end{aligned}
$$

where $H(0)$ follows from Eq. (A3) and $I_{\ell \text {,disp }}\left(I_{\ell, \text { abs }}\right)$ indicate the dispersive (absorptive) parts of the two-loop diagrams. The $\ell=e$ case is dominated by the absorptive contribution: $I_{e, \text { disp }} \simeq 1.4, I_{e, \text { abs }} \simeq-35$. This gives $R_{S}^{e}=7.8 \times 10^{-9}$ corresponding to

$$
\operatorname{BR}\left(K_{S} \rightarrow e^{+} e^{-}\right)=2.1 \times 10^{-14}
$$

to be compared with the recent bound by Ambrosino et al. (2009b):

$$
\operatorname{BR}\left(K_{S} \rightarrow e^{+} e^{-}\right)<9 \times 10^{-9} \quad(90 \% \text { C.L. }) .
$$

For $\ell=\mu$ there is a slight dominance of the dispersive contribution, $I_{\mu, \text { disp }} \simeq-2.8, I_{\mu, \text { abs }} \simeq 1.2$, which implies $R_{S}^{\mu}=1.9 \times 10^{-6}$ and

$$
\operatorname{BR}\left(K_{S} \rightarrow \mu^{+} \mu^{-}\right)=5.1 \times 10^{-12},
$$

to be compared with the (almost 30-year-old) bound (Gjesdal et al. 1973)

$$
\left.\operatorname{BR}\left(K_{S} \rightarrow \mu^{+} \mu^{-}\right)<3.2 \times 10^{-7} \quad \text { (90\% C.L. }\right) .
$$

There exists a small short-distance contribution to this decay through the $\mathrm{CP}$-violating component of the s-wave amplitude $C$ in Eq. 6.35, which in the SM was estimated for the muon case as (Buchalla et al., 1996, Isidori and Unterdorfer, 2004)

$$
C_{\mathrm{sd}}=-\frac{G_{F} \alpha\left(M_{Z}\right)}{\pi \sin ^{2} \theta_{W}} \sqrt{2} m_{\mu} F_{K} \operatorname{Im}\left(V_{t s}^{*} V_{t d}\right) Y\left(x_{t}\right),
$$

where $Y\left(x_{t}\right)$ is given in Buchalla et al. (1996). Using the Wolfenstein parametrization, the short-distance contribution to the branching ratio is given by

$$
\begin{aligned}
\operatorname{BR}\left(K_{S} \rightarrow \mu^{+} \mu^{-}\right)_{\mathrm{sd}} & =1.4 \times 10^{-12}\left|\frac{V_{c b}}{0.041}\right|^{4}\left|\frac{\lambda}{0.225}\right|^{2} \bar{\eta}^{2} \\
& \simeq 1.7 \times 10^{-13},
\end{aligned}
$$

an order of magnitude short of the CP-invariant contribution.

\section{2. $K_{L} \rightarrow \ell^{+} \ell^{-}$}

If $\mathrm{CP}$ invariance is preserved this decay is given by the amplitude $C$ in Eq. (6.34). It is convenient to normalize the rate to the $K_{L} \rightarrow \gamma \gamma$ mode:

$$
\begin{aligned}
R_{L}^{\ell} & =\frac{\Gamma\left(K_{L} \rightarrow \ell^{+} \ell^{-}\right)}{\Gamma\left(K_{L} \rightarrow \gamma \gamma\right)} \\
& =2 \beta_{\ell}\left(\frac{\alpha}{\pi} r_{\ell}\right)^{2}\left(\left|F_{\ell, \text { disp }}\right|^{2}+\left|F_{\ell, \text { abs }}\right|^{2}\right)
\end{aligned}
$$

The absorptive amplitude gets contributions from several available on-shell states (Martin et al. 1970), but the $\gamma \gamma$ intermediate state dominates completely (Fig. 12):

$$
F_{\ell, \text { abs }}=\frac{\pi}{2 \beta_{\ell}} \ln \left(\frac{1-\beta_{\ell}}{1+\beta_{\ell}}\right) \text {. }
$$

The dispersive part arises from the one-loop diagram $K_{L} \rightarrow \gamma^{*} \gamma^{*} \rightarrow \mu^{+} \mu^{-}$and local CHPT terms to absorb the loop divergence (Gómez Dumm and Pich, 1998, Isidori and Unterdorfer, 2004, Knecht et al. 1999a):

$$
\begin{aligned}
F_{\ell, \text { disp }}= & \frac{1}{4 \beta_{\ell}} \ln ^{2}\left(\frac{1-\beta_{\ell}}{1+\beta_{\ell}}\right)+\frac{1}{\beta_{\ell}} \operatorname{Li}_{2}\left(\frac{\beta_{\ell}-1}{\beta_{\ell}+1}\right)+\frac{\pi^{2}}{12 \beta_{\ell}} \\
& +3 \ln \frac{m_{\ell}}{\mu}+\chi(\mu),
\end{aligned}
$$

where the local contribution can be split into its longdistance and short-distance components:

$$
\chi(\mu)=\chi_{\gamma \gamma}(\mu)+\chi_{s d} .
$$

$\chi_{\gamma \gamma}(\mu)$ compensates the scale dependence of the one-loop amplitude, while $\chi_{s d}$ accounts for the short-distance contribution $\bar{s} d \rightarrow \mu^{+} \mu^{-}$.

The $K_{L} \rightarrow \mu^{+} \mu^{-}$decay is well established experimentally. The measured rate (Nakamura et al. 2010$)$

$$
R_{L}^{\mu}=(1.25 \pm 0.02) \times 10^{-5}
$$

appears to be nearly saturated by the absorptive contribution $6.44,\left.R_{L}^{\mu}\right|_{\text {abs }}=1.195 \times 10^{-5}$. The difference between these two numbers provides an experimental measurement of the modulus of the dispersive amplitude and, therefore, up to a two-fold ambiguity, of the local term:

$$
\chi\left(M_{\rho}\right)=\left\{\begin{array}{l}
3.75 \pm 0.20 \\
1.52 \pm 0.20
\end{array} .\right.
$$

The $K_{L} \rightarrow e^{+} e^{-}$rate is then predicted to be $R_{L}^{e}=$ $(1.552 \pm 0.014) \times 10^{-8}$ or $(1.406 \pm 0.013) \times 10^{-8}$, respectively. Both values are in agreement with the present experimental result $\left.R_{L}^{e}\right|_{\exp }=(1.65 \pm 0.91) \times 10^{-8}$ brose et al. 1998), obtained with only four events. Incidentally, this is at present the tiniest branching ratio ever measured: $\operatorname{BR}\left(K_{L} \rightarrow e^{+} e^{-}\right)=9_{-4}^{+6} \times 10^{-12}$. 
The corresponding branching ratio for the muon case is $\operatorname{BR}\left(K_{L} \rightarrow \mu^{+} \mu^{-}\right)=(6.84 \pm 0.11) \times 10^{-9}$ Ambrose et al. 2000).

At $\mathcal{O}\left(p^{6}\right)$ in the chiral expansion, the $K_{L} \rightarrow \gamma \gamma$ amplitude is given by the $\pi^{0}, \eta, \eta^{\prime}$ exchange mechanism shown in Fig. 11. The details of the weak transition vertex cancel out in the ratio $R_{L}^{\ell}$. Therefore, removing the shortdistance contribution $\chi_{s d}$, Eqs. 6.43 to 6.45 can be directly applied to the electromagnetic $2 \gamma$ decays of the neutral unflavored pseudoscalar mesons. Moreover, the local CHPT contributions are also the same. Thus, from the measured $\pi^{0} \rightarrow e^{+} e^{-}$and $\eta \rightarrow \mu^{+} \mu^{-}$rates one can determine $\chi_{\gamma \gamma}(\mu)$ (Gómez Dumm and Pich, 1998), again up to a two-fold ambiguity. One gets $\chi_{\gamma \gamma}\left(M_{\rho}\right)=0.8 \pm 1.0$ or $-20.7 \pm 1.0$ from $\pi^{0} \rightarrow e^{+} e^{-}$, and $\chi_{\gamma \gamma}\left(M_{\rho}\right)=5.5 \pm 0.9$ or $-0.8 \pm 0.9$ from $\eta \rightarrow \mu^{+} \mu^{-}$. The second $\pi^{0} \rightarrow e^{+} e^{-}$ solution is clearly excluded. The first one agrees very well with the negative $\eta \rightarrow \mu^{+} \mu^{-}$solution, but it is only $3.2 \sigma$ away from the positive one. Keeping the two alternative possibilities and following the PDG average prescription, we get

$$
\chi_{\gamma \gamma}\left(M_{\rho}\right)=\left\{\begin{array}{c}
-0.1 \pm 0.7 \\
3.4 \pm 2.3
\end{array},\right.
$$

to be compared with the theoretical estimate $\chi_{\gamma \gamma}\left(M_{\rho}\right)=$ $-0.3 \pm 0.9$ (Knecht et al. 1999a), evaluated within lowestmeson dominance in the large- $N_{C}$ framework. An alternative theoretical estimate was obtained (D'Ambrosio et al., 1998b; Isidori and Unterdorfer, 2004) performing the $2 \gamma$ loop integration with the form factor (6.33), with $\alpha_{\text {DIP }}=-\left(1+\beta_{\text {DIP }}\right) / 2=-1.69 \pm 0.08$ as determined from the radiative decays. One gets in this way $\chi_{\gamma \gamma}\left(M_{\rho}\right)=3.3 \pm 1.3$, also compatible with 6.49).

The short-distance contribution to $K_{L} \rightarrow \mu^{+} \mu^{-}$is well known at NLO (Buchalla et al. 1996) and a NNLO evaluation of the charm-quark contribution $Y_{\mathrm{NL}}$ was carried out more recently (Gorbahn and Haisch, 2006). The relative sign with respect to the long-distance amplitude can be fixed unambiguously in the large- $N_{C}$ limit Gómez Dumm and Pich, 1998, Isidori and Unterdorfer, 2004):

$$
\begin{aligned}
\chi_{s d}^{\mathrm{SM}} & =4.965 \times 10^{3}\left[\operatorname{Re}\left(\lambda_{t}\right) Y\left(x_{t}\right)+\operatorname{Re}\left(\lambda_{c}\right) Y_{\mathrm{NL}}\right] \\
& =-1.82 \pm 0.04 .
\end{aligned}
$$

Adding the long-distance component 6.49), one gets $\chi\left(M_{\rho}\right)=-1.9 \pm 0.7$ or $1.6 \pm 2.3$, to be compared with Eq. (6.48). Although the present uncertainties are still too large to make a meaningful test of $\chi_{s d}$, a better understanding of the long-distance amplitude could well uncover new-physics contributions.

The longitudinal polarization $P_{L}$ of either muon in the decay $K_{L} \rightarrow \mu^{+} \mu^{-}$is a measure of CP violation (Pais and Treiman, 1968b: Sehgal, 1969). Within the SM, the main source for $P_{L}$ is indirect CP violation due to $K^{0}-$ $\overline{K^{0}}$ mixing. The longitudinal polarization arises from the interference of both amplitudes in Eq. 6.34):

$$
P_{L}=\frac{N_{R}-N_{L}}{N_{R}+N_{L}}=-\frac{M_{K} \beta_{\mu}^{2}}{4 \pi \Gamma} \operatorname{Im}\left(B C^{*}\right),
$$

where $N_{R}, N_{L}$ are the numbers of outgoing $\mu^{-}$with positive or negative helicity, respectively and $\Gamma$ is the full decay width.

Within the SM, one expects (Ecker and Pich, 1991)

$$
\left|P_{L}\right|=(2.6 \pm 0.4) \times 10^{-3} .
$$

This polarization has not been measured yet.

D. $K \rightarrow \pi \gamma \gamma^{(*)}$

The general amplitude for $K(k) \rightarrow \pi(p) \gamma\left(q_{1}\right) \gamma\left(q_{2}\right)$,

$$
A(K \rightarrow \pi \gamma \gamma)=\varepsilon_{\mu}^{*}\left(q_{1}\right) \varepsilon_{\nu}^{*}\left(q_{2}\right) M^{\mu \nu}\left(k, q_{1}, q_{2}\right),
$$

contains four Lorentz structures:

$$
\begin{aligned}
M^{\mu \nu}= & \frac{A(z, y)}{M_{K}^{2}}\left(q_{2}^{\mu} q_{1}^{\nu}-q_{1} \cdot q_{2} g^{\mu \nu}\right) \\
+ & \frac{2 B(z, y)}{M_{K}^{4}}\left(-k \cdot q_{1} k \cdot q_{2} g^{\mu \nu}-q_{1} \cdot q_{2} k^{\mu} k^{\nu}\right. \\
& \left.\quad+k \cdot q_{1} q_{2}^{\mu} k^{\nu}+k \cdot q_{2} k^{\mu} q_{1}^{\nu}\right) \\
+ & \frac{C(z, y)}{M_{K}^{2}} \varepsilon^{\mu \nu \rho \sigma} q_{1 \rho} q_{2 \sigma} \\
+ & \frac{D(z, y)}{M_{K}^{4}}\left[\varepsilon^{\mu \nu \rho \sigma}\left(k \cdot q_{2} q_{1 \rho}+k \cdot q_{1} q_{2 \rho}\right) k_{\sigma}\right. \\
& \left.+\left(k^{\mu} \varepsilon^{\nu \alpha \beta \gamma}+k^{\nu} \varepsilon^{\mu \alpha \beta \gamma}\right) k_{\alpha} q_{1 \beta} q_{2 \gamma}\right],
\end{aligned}
$$

where $z=\left(q_{1}+q_{2}\right)^{2} / M_{K}^{2}$ and $y=k \cdot\left(q_{1}-q_{2}\right) / M_{K}^{2}$. Bose symmetry requires the invariant amplitudes $A(z, y)$, $B(z, y)$ and $C(z, y)$ to be even in $y$, while $D(z, y)$ is odd. In the limit where $\mathrm{CP}$ is conserved, $A$ and $B$ contribute only to $K_{L} \rightarrow \pi^{0} \gamma \gamma$, while $C$ and $D$ contribute to $K_{S} \rightarrow$ $\pi^{0} \gamma \gamma$. All of them are involved in $K^{+} \rightarrow \pi^{+} \gamma \gamma$.

The double differential rate for unpolarized photons is

$$
\begin{aligned}
\frac{d^{2} \Gamma}{d y d z}=\frac{M_{K}}{2^{9} \pi^{3}} & \left\{z^{2}\left(|A+B|^{2}+|C|^{2}\right)\right. \\
+ & {\left.\left[y^{2}-\frac{1}{4} \lambda\left(1, r_{\pi}^{2}, z\right)\right]^{2}\left(|B|^{2}+|D|^{2}\right)\right\} . }
\end{aligned}
$$

The physical region is given by $0 \leq|y| \leq \lambda^{1 / 2}\left(1, r_{\pi}^{2}, z\right) / 2$ and $0 \leq z \leq\left(1-r_{\pi}\right)^{2}$.

The processes $K \rightarrow \pi \gamma \gamma$ have no tree-level contributions of $\mathcal{O}\left(p^{2}\right)$. At $\mathcal{O}\left(p^{4}\right)$ the amplitudes $B$ and $D$ are still zero, since there are not enough powers of momenta to generate the gauge structure. Therefore, $B$ and $D$ arise only at $\mathcal{O}\left(p^{6}\right)$. Notice that both $B$ and $D$ lead to contributions also for small $z$.

The amplitudes for $K \rightarrow \pi \gamma \gamma^{*}$ have a related but much more involved structure than with both photons on-shell. We comment briefly on these processes. 

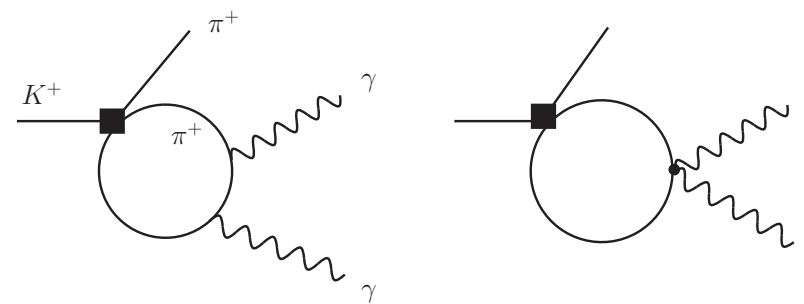

FIG. 13 Unitarity contribution from $K^{+} \rightarrow \pi^{+} \pi^{+} \pi^{-}$to $K^{+} \rightarrow \pi^{+} \gamma \gamma$.

1. $K^{+} \rightarrow \pi^{+} \gamma \gamma$

The leading contribution of $\mathcal{O}\left(p^{4}\right)$ to the dominant octet amplitude for $K^{+} \rightarrow \pi^{+} \gamma \gamma$ was determined in Ecker et al. (1988):

$$
\begin{aligned}
& A^{(4)}(z)=\frac{G_{8} M_{K}^{2} \alpha}{2 \pi z} {\left[\left(z+1-r_{\pi}^{2}\right) F\left(z / r_{\pi}^{2}\right)\right.} \\
&\left.+\left(z-1+r_{\pi}^{2}\right) F(z)-\hat{c} z\right]
\end{aligned}
$$

where $F(z)$ is defined in Eq. A1 and

$$
\hat{c}=128 \pi^{2}\left[3\left(L_{9}+L_{10}\right)+N_{14}-N_{15}-2 N_{18}\right] / 3
$$

in terms of the LECs in Eqs. 22.15, 2.16). Note that the loop contribution is finite and, consequently, the counterterm combination is scale independent. The subdominant 27-plet contribution to the amplitude $A^{(4)}(z)$ was also determined (Gérard et al., 2005). In addition, starting at $\mathcal{O}\left(p^{4}\right)$ but going beyond it,

$$
C(z)=\frac{G_{8} M_{K}^{2} \alpha}{\pi}\left[\frac{z-r_{\pi}^{2}}{z-r_{\pi}^{2}+i r_{\pi} \frac{\Gamma_{\pi 0}}{M_{K}}}-\frac{3 z-2-r_{\pi}^{2}}{3\left(z-r_{\eta}^{2}\right)}\right],
$$

where $r_{\eta}=M_{\eta} / M_{K}$ and $\Gamma_{\pi^{0}} \equiv \Gamma\left(\pi^{0} \rightarrow \gamma \gamma\right)$. Notice that the imaginary part of the $\eta$ pole has not been included. This amplitude is generated by the WessZumino-Witten functional in Eq. 2.15 through the sequence $K^{+} \rightarrow \pi^{+}\left(\pi^{0}, \eta\right) \rightarrow \pi^{+} \gamma \gamma$. The $\eta^{\prime}$ contribution, though fairly suppressed by its mass, was also considered within U(3) CHPT (Gérard et al. 2005).

Although there is no complete evaluation to $\mathcal{O}\left(p^{6}\right)$, the most important contributions have been estimated. The unitarity corrections from $K^{+} \rightarrow \pi^{+} \pi^{+} \pi^{-}$, shown in Fig. 13 were determined by D'Ambrosio and Portolés (1996). They contribute to both amplitudes $A(z, y)$ and $B(z)$, where $A$ becomes $y$-dependent. Local non-resonant contributions were also studied and a naive chiral dimensional estimate indicates that they are small. Vectorresonance contributions to the $\mathcal{O}\left(p^{6}\right)$ LECs are introduced through their effect on the amplitude $B$ (Cohen et al. 1993):

$$
a_{V}^{+}=-\frac{\pi}{2 G_{8} M_{K}^{2} \alpha} \lim _{z \rightarrow 0} B_{V}(z) .
$$

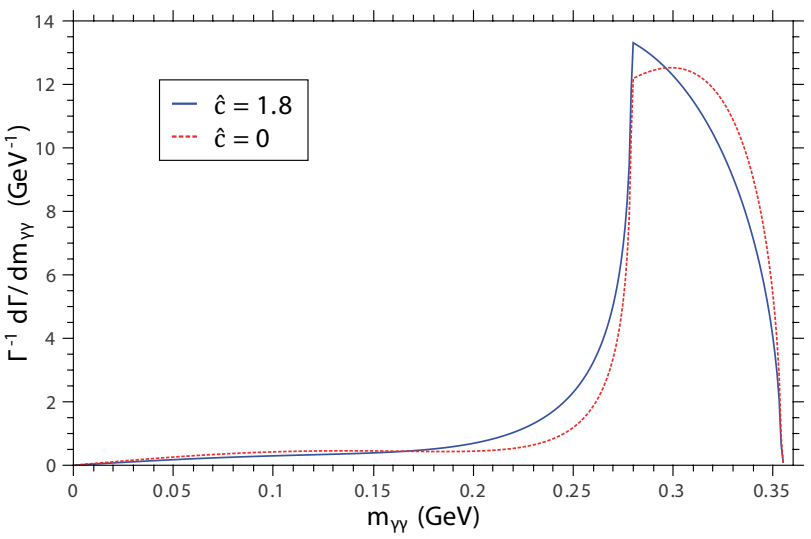

FIG. 14 Predicted spectra in the diphoton invariant mass at different values of $\hat{c}$ for $K^{+} \rightarrow \pi^{+} \gamma \gamma$.

The vector-exchange contribution to $A$ can also be written in terms of the parameter $a_{V}^{+}$, if we assume that these local amplitudes are generated through strong resonance exchange supplemented with a weak transition in the external legs (Ecker et al. 1990):

$$
A_{V}=G_{8} M_{K}^{2} \alpha a_{V}^{+}\left(3+r_{\pi}^{2}-z\right) / \pi
$$

These vector-exchange contributions were estimated by D'Ambrosio and Portolés (1997) and found to be very small: $a_{V}^{+}=-0.2 \pm 0.3$.

The branching ratio of this decay and its spectrum were measured by Kitching et al. (1997):

$$
\operatorname{BR}\left(K^{+} \rightarrow \pi^{+} \gamma \gamma\right)=(1.1 \pm 0.3) \times 10^{-6} .
$$

Hence it is possible to determine the value of $\hat{c}$ in Eq. 6.56). From the analysis of the normalized spectrum at $\mathcal{O}\left(p^{4}\right)$ the value $\hat{c}=1.6 \pm 0.6\left(\chi^{2} /\right.$ dof $\left.=0.9\right)$ is obtained. Including the unitarity corrections and neglecting local $\mathcal{O}\left(p^{6}\right)$ contributions, the fit improves $\left(\chi^{2} /\right.$ dof $\left.=0.7\right)$ yielding $\hat{c}=1.8 \pm 0.6$. The diphoton invariant-mass distribution is shown in Fig. 14 for different values of $\hat{c}$. The spectrum observed by NA48/2 is shown in Fig. 15.

The electromagnetic penguin operators induce a CPviolating charge asymmetry in $K^{ \pm} \rightarrow \pi^{ \pm} \gamma \gamma$. This asymmetry is estimated to be tiny within the SM (Ecker et al. 1988; Gao, 2003).

\section{2. $K_{S} \rightarrow \pi^{0} \gamma \gamma$}

The leading contribution to this decay is given by $K^{0} \rightarrow \pi^{0}\left(\pi^{0}, \eta\right) \rightarrow \pi^{0} \gamma \gamma$, where the first part is dominated by the octet weak transition in the Lagrangian (2.16) and the second part arises from the Wess-ZuminoWitten anomalous term in Eq. (2.15). The amplitude starts at $\mathcal{O}\left(p^{4}\right)$ but includes also higher orders (Ecker 


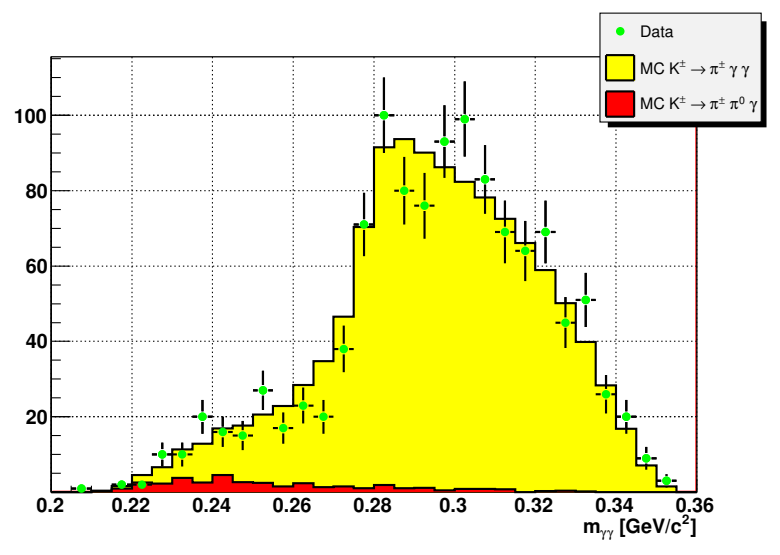

FIG. 15 Spectrum for $K^{+} \rightarrow \pi^{+} \gamma \gamma$ in the diphoton invariant mass by NA48/2. From Morales (2008).

et al. 1987b):

$C(z)=\frac{G_{8} M_{K}^{2} \alpha}{\pi}\left[\frac{2-z-r_{\pi}^{2}}{z-r_{\pi}^{2}+i r_{\pi} \frac{\Gamma_{\pi 0}}{M_{K}}}-\frac{F_{\pi}\left(2-3 z+r_{\pi}^{2}\right)}{3 F_{\eta}\left(z-r_{\eta}^{2}\right)}\right]$,

keeping $F_{\pi} \neq F_{\eta}$ to account for chiral corrections. An amplitude $D(z, y)$ would arise at higher chiral orders. As in the $K^{ \pm}$decay, the $\eta^{\prime}$ contribution was also included by Gérard et al. (2005). It is seen, however, that the amplitude is dominated by the pion pole.

In order to eliminate the overwhelming background from $K_{S} \rightarrow \pi^{0} \pi^{0}$, one restricts the kinematical region to $z>0.2$. The chiral prediction gives

$$
\operatorname{BR}\left(K_{S} \rightarrow \pi^{0} \gamma \gamma\right)_{z>0.2}=3.8 \times 10^{-8}
$$

that compares well with the experimental measurement (Lai et al., 2004)

$$
\operatorname{BR}\left(K_{S} \rightarrow \pi^{0} \gamma \gamma\right)_{z>0.2}=(4.9 \pm 1.8) \times 10^{-8} .
$$

\section{3. $K_{L} \rightarrow \pi^{0} \gamma \gamma$}

The relevance of this channel extends beyond its own interest as it provides a $\mathrm{CP}$-conserving contribution via the two-photon cut to the decay $K_{L} \rightarrow \pi^{0} \ell^{+} \ell^{-}$ (Donoghue et al., 1987; Ecker et al., 1988; Heiliger and Sehgal 1993a Sehgal 1988) that competes with direct and indirect $\mathrm{CP}$-violating contributions.

The absence of $\mathcal{O}\left(p^{4}\right)$ counterterms for this process indicates that the only contribution is a finite one-loop result at this order (Cappiello and D'Ambrosio, 1988, Ecker et al. 1987b Sehgal, 1990). The octet part of the amplitude $A(z)$ is

$$
\begin{aligned}
A^{(4)}(z)=\frac{G_{8} M_{K}^{2} \alpha}{\pi z} & {\left[\left(z-r_{\pi}^{2}\right) F\left(z / r_{\pi}^{2}\right)\right.} \\
& \left.-\left(z-1-r_{\pi}^{2}\right) F(z)\right],
\end{aligned}
$$

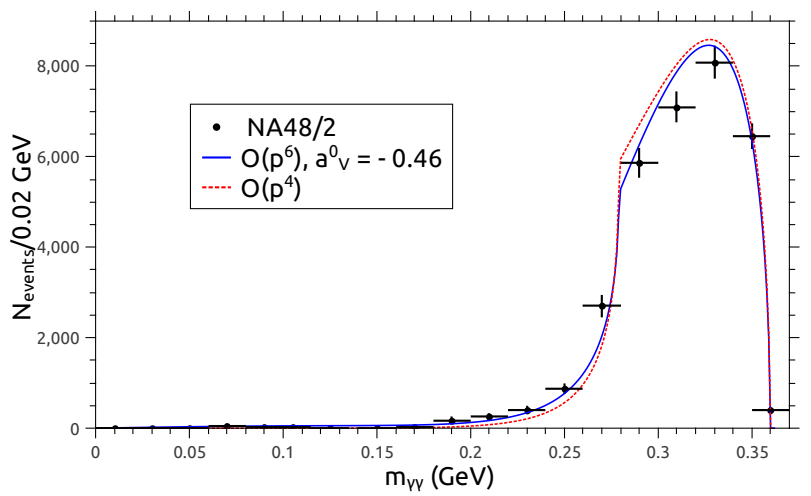

FIG. 16 Comparison of the normalized spectra in the diphoton invariant mass for $K_{L} \rightarrow \pi^{0} \gamma \gamma$ at $\mathcal{O}\left(p^{4}\right)$ and $\mathcal{O}\left(p^{6}\right)$ in CHPT. The data are from Lai et al. (2002).

where $F(z)$ is given in Eq. A1). This result gives rise to $\operatorname{BR}\left(K_{L} \rightarrow \pi^{0} \gamma \gamma\right)=6.8 \times 10^{-1}$, significantly smaller than the present PDG average (Nakamura et al., 2010)

$$
\operatorname{BR}\left(K_{L} \rightarrow \pi^{0} \gamma \gamma\right)=(1.27 \pm 0.03) \times 10^{-6} .
$$

This indicates that higher-order chiral corrections should be considered.

The unitarity corrections from $K_{L} \rightarrow \pi^{+} \pi^{-} \pi^{0}$ contributing at $\mathcal{O}\left(p^{6}\right)$ (Cappiello et al. 1993 , Cohen et al. $1993)$ give rise to both $A$ and $B$ amplitudes. The inclusion of the 27-plet contribution was carried out by Cappiello et al. (1993). As in the $K^{ \pm}$case, those amplitudes also get local contributions from both vector exchange and non-resonant parts. From dimensional analysis the latter seem to be negligible, but the situation of vector resonance contributions is still not settled. This is parametrized by $a_{V}^{0}$, defined as in Eq. 6.59), that was estimated theoretically to take the value $a_{V}^{0}=-0.7 \pm 0.3$ (D'Ambrosio and Portolés, 1997). The measured spectrum clearly favors a non-zero value for this parameter: NA48 (Lai et al., 2002) gets $a_{V}^{0}=-0.46 \pm 0.05$ while $\mathrm{KTeV}$ (Abouzaid et al. 2008b) obtains $a_{V}^{0}=$ $-0.31 \pm 0.09$. The predicted spectrum is compared with the measured distribution in Fig. 16 .

On the basis of the experimental situation at the time, Gabbiani and Valencia (2002) argued that the complete set of three $\mathcal{O}\left(p^{6}\right)$ counterterms is needed to describe both branching ratio and spectrum. However, the latest experimental analyses (Abouzaid et al., 2008b; Lai et al., 2002 find a satisfactory representation in terms of $a_{V}^{\mathrm{v}}$ only, supporting the dominance of vector exchange.

\section{4. $K \rightarrow \pi \gamma \ell^{+} \ell^{-}$}

These decays are obviously mediated by $K \rightarrow \pi \gamma \gamma^{*}$ and are closely related to the case with both photons onshell. The study of $K^{+} \rightarrow \pi^{+} \gamma \ell^{+} \ell^{-}$and $K_{L} \rightarrow \pi^{0} \gamma \ell^{+} \ell^{-}$ was carried out by Donoghue and Gabbiani (1997, 1998) and Gabbiani (1999). 
The decay amplitude has a more complex Lorentz structure than in Eq. (6.54). Nevertheless, at $\mathcal{O}\left(p^{4}\right)$ these decays have the same properties as $K^{+} \rightarrow \pi^{+} \gamma \gamma$ and $K_{L} \rightarrow \pi^{0} \gamma \gamma$, i.e., both have finite one-loop contributions, the charged channel has the same scale-independent counterterm $\hat{c}$ and the neutral one has no local contributions. Taking $\hat{c}=1.8$, one obtains at this order:

$$
\begin{aligned}
\operatorname{BR}\left(K^{+} \rightarrow \pi^{+} \gamma e^{+} e^{-}\right) & =1.4 \times 10^{-8}, \\
\operatorname{BR}\left(K_{L} \rightarrow \pi^{0} \gamma e^{+} e^{-}\right) & =1.0 \times 10^{-8} .
\end{aligned}
$$

These predictions have to be compared with the recent experimental measurements (Batley et al. 2008a)

$$
\mathrm{BR}\left(K^{+} \rightarrow \pi^{+} \gamma e^{+} e^{-}\right)=(1.29 \pm 0.13) \times 10^{-8}
$$

and (Abouzaid et al. 2007a)

$$
\mathrm{BR}\left(K_{L} \rightarrow \pi^{0} \gamma e^{+} e^{-}\right)=(1.62 \pm 0.17) \times 10^{-8} .
$$

As can be seen, the agreement is rather good in the charged-kaon channel.

Unitarity corrections from $K \rightarrow \pi \pi \pi$, extending beyond $\mathcal{O}\left(p^{4}\right)$, were performed for both decays. We comment on them in turn.

- $K^{+} \rightarrow \pi^{+} \gamma e^{+} e^{-}$: Local $\mathcal{O}\left(p^{6}\right)$ counterterms were found to be negligible in $K^{+} \rightarrow \pi^{+} \gamma \gamma$. However, neglecting them also in this case and using $\hat{c}=1.8$, one finds $\operatorname{BR}\left(K^{+} \rightarrow \pi^{+} \gamma e^{+} e^{-}\right)=1.7 \times 10^{-8}$, which disagrees with the experimental result. Indeed, the analysis of the spectrum (for $m_{e e \gamma}>260 \mathrm{MeV}$ ) carried out in Batley et al. (2008a) gets $\hat{c}=0.90 \pm 0.45$. Hence local contributions of $\mathcal{O}\left(p^{6}\right)$ and beyond might be non-negligible after all.

- $K_{L} \rightarrow \pi^{0} \gamma e^{+} e^{-}$: Together with unitarity corrections, Donoghue and Gabbiani (1997) included explicit contributions from vector resonances. Their prediction $\operatorname{BR}\left(K_{L} \rightarrow \pi^{0} \gamma e^{+} e^{-}\right)=2.3 \times 10^{-8}$ is in slight disagreement with the experimental measurement quoted above. Abouzaid et al. (2007a) concluded that employing a value of $a_{V}^{v}=-0.46$ (in accordance with $K_{L} \rightarrow \pi^{0} \gamma \gamma$ ), the branching ratio goes down to $\operatorname{BR}\left(K_{L} \rightarrow \pi^{0} \gamma e^{+} e^{-}\right)=1.51 \times 10^{-8}$, in better agreement with the measured width.

\section{E. $K \rightarrow \pi \ell^{+} \ell^{-}$}

The FCNC transitions $K \rightarrow \pi \ell^{+} \ell^{-}(\ell=e, \mu)$ are dominated by single virtual-photon exchange $\left(K \rightarrow \pi \gamma^{*}\right)$ if allowed by CP invariance as in $K_{S}$ and $K^{ \pm}$decays. This contribution is CP violating for $K_{L} \rightarrow \pi^{0} \ell^{+} \ell^{-}$and consequently this process has become a point of reference for studying the CP-violating sector of the SM.

\section{1. $K_{S}, K^{ \pm} \rightarrow \pi \ell^{+} \ell^{-}$}

The amplitude for $K(k) \rightarrow \pi(p) \ell^{+}\left(p_{+}\right) \ell^{-}\left(p_{-}\right)$is determined by an electromagnetic transition form factor

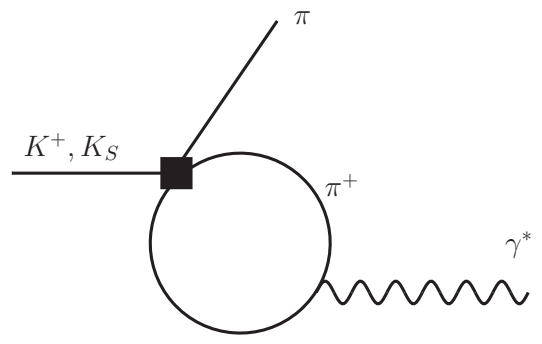

FIG. 17 Unitarity $K \rightarrow \pi \pi \pi$ contribution to $K^{+}, K_{S} \rightarrow \pi \gamma^{*}$.

in the presence of the nonleptonic weak interactions (D'Ambrosio et al., 1998a; Ecker et al., 1987a):

$$
A=-\frac{G_{F} \alpha}{4 \pi} V(z)(k+p)^{\mu} \bar{u}_{\ell}\left(p_{-}\right) \gamma_{\mu} v_{\ell}\left(p_{+}\right),
$$

where $z=q^{2} / M_{K}^{2}$ and $q=k-p$. The spectrum in the dilepton invariant mass is given by

$$
\frac{d \Gamma}{d z}=\frac{G_{F}^{2} \alpha^{2} M_{K}^{5}}{12 \pi(4 \pi)^{4}} \bar{\lambda}^{3 / 2} \sqrt{1-4 \frac{r_{\ell}^{2}}{z}}\left(1+2 \frac{r_{\ell}^{2}}{z}\right)|V(z)|^{2},
$$

where $\bar{\lambda} \equiv \lambda\left(1, z, r_{\pi}^{2}\right)$ and $4 r_{\ell}^{2} \leq z \leq\left(1-r_{\pi}\right)^{2}$.

Because of gauge invariance $V(z)=0$ at $\mathcal{O}\left(p^{2}\right)$. The first evaluation at $\mathcal{O}\left(p^{4}\right)$ of the dominant octet amplitude was carried out by Ecker et al. (1987a):

$$
V_{+}(z)=-\frac{G_{8}}{G_{F}}\left[\Phi(z)+\Phi\left(z / r_{\pi}^{2}\right)+w_{+}\right]
$$

for $K^{ \pm} \rightarrow \pi^{ \pm} \gamma^{*}$, and

$$
V_{S}(z)=\frac{G_{8}}{G_{F}}\left[2 \Phi(z)+w_{S}\right]
$$

for $K_{S} \rightarrow \pi^{0} \gamma^{*}$. Here

$$
\Phi(z)=\frac{5}{18}-\frac{4}{3 z}+\frac{4}{3 z}\left(1-\frac{z}{4}\right) G(z),
$$

where $G(z)$ is given in Eq. A2). In terms of the LECs in Eqs. 2.15 and 2.16, the local $\mathcal{O}\left(p^{4}\right)$ contributions are

$$
\begin{aligned}
& w_{+}=\frac{64 \pi^{2}}{3}\left(N_{14}^{r}-N_{15}^{r}+3 L_{9}^{r}\right)+\frac{1}{3} \ln \frac{\mu^{2}}{M_{K} M_{\pi}}, \\
& w_{S}=\frac{32 \pi^{2}}{3}\left(2 N_{14}^{r}+N_{15}^{r}\right)+\frac{1}{3} \ln \frac{\mu^{2}}{M_{K}^{2}} .
\end{aligned}
$$

Although a complete study to $\mathcal{O}\left(p^{6}\right)$ has not been performed yet, the unitarity corrections from $K \rightarrow \pi \pi \pi$, shown in Fig. 17, were determined by D'Ambrosio et al. (1998a). The full result can be decomposed in a polynomial contribution (linear in $z$ up to this order) plus the unitarity loop corrections,

$$
V_{i}(z)=a_{i}+b_{i} z+V_{i}^{\pi \pi}(z) \quad(i=+, S),
$$


the latter being given by

$$
V_{i}^{\pi \pi}(z)=\frac{\alpha_{i}+\beta_{i}\left(z-z_{0}\right) / r_{\pi}^{2}}{G_{F} M_{K}^{2} r_{\pi}^{2}}\left[1+\frac{z}{r_{V}^{2}}\right]\left[\Phi\left(z / r_{\pi}^{2}\right)+\frac{1}{6}\right],
$$

with $z_{0}=r_{\pi}^{2}+1 / 3, r_{V}=M_{\rho} / M_{K}$ and

$$
\begin{aligned}
\alpha_{+} & =\beta_{1}-\frac{1}{2} \beta_{3}+\sqrt{3} \gamma_{3}, & \beta_{+} & =2\left(\xi_{1}+\xi_{3}-\xi_{3}^{\prime}\right), \\
\alpha_{S} & =-\frac{4}{\sqrt{3}} \gamma_{3}, & \beta_{S} & =\frac{8}{3} \xi_{3}^{\prime},
\end{aligned}
$$

in terms of the parameters in Eq. (5.33). The parametrization (6.76) includes the $\mathcal{O}\left(p^{4}\right)$ contributions. The polynomial part incorporates the local counterterms in Eq. 6.75 through $a_{+}^{(4)}=G_{8} / G_{F}\left(1 / 3-w_{+}\right)$and $a_{S}^{(4)}=-G_{8} / G_{F}\left(1 / 3-w_{S}\right)$, and tiny contributions to the slopes $b_{i}^{(4)}$ from the $\mathcal{O}\left(p^{4}\right)$ kaon loop.

$V_{i}(z)$ in Eq. 6.76) is expected to be an excellent approximation to the complete form factor of $\mathcal{O}\left(p^{6}\right)$. It only assumes that all contributions except the two-pion intermediate state can be well approximated by a linear polynomial for small values of $z$. The predicted rates can then be expressed in terms of $a_{i}$ and $b_{i}$ in Eq. (6.76):

$$
\begin{aligned}
\mathrm{BR}_{K^{ \pm}}^{e}=[ & 0.15-3.31 a_{+}-0.90 b_{+}+60.51 a_{+}^{2} \\
& \left.+16.36 a_{+} b_{+}+1.77 b_{+}^{2}\right] \times 10^{-8}, \\
\mathrm{BR}_{K^{ \pm}}^{\mu}=[ & 1.19-19.97 a_{+}-6.56 b_{+}+120.16 a_{+}^{2} \\
& \left.+69.42 a_{+} b_{+}+10.59 b_{+}^{2}\right] \times 10^{-9}, \\
\mathrm{BR}_{K_{S}}^{e}=[ & 0.01-0.55 a_{S}-0.17 b_{S}+43.76 a_{S}^{2} \\
& \left.+11.83 a_{S} b_{S}+1.28 b_{S}^{2}\right] \times 10^{-10}, \\
\mathrm{BR}_{K_{S}}^{\mu}=[ & 0.07-3.96 a_{S}-1.34 b_{S}+86.90 a_{S}^{2} \\
& \left.+50.21 a_{S} b_{S}+7.66 b_{S}^{2}\right] \times 10^{-11} .
\end{aligned}
$$

If $a_{i}, b_{i} \sim 1$ as expected, the polynomial contribution dominates over the unitarity-cut loop corrections coming from $K \rightarrow \pi \pi \pi$. In particular, due to the strong suppression of $K_{S} \rightarrow \pi^{+} \pi^{-} \pi^{0}$, the latter are tiny for the $K_{S}$ decay. Thus, these decays are very sensitive to the chiral LECs.

The values of $a_{+}$and $b_{+}$have been fitted from the $K^{+} \rightarrow \pi^{+} \ell^{+} \ell^{-}$spectra. One finds $a_{+}=-0.578 \pm 0.016$ and $b_{+}=-0.779 \pm 0.066$ for $\ell=e$ (Batley et al., 2009b), while the muonic mode gives $a_{+}=-0.575 \pm 0.039$ and $b_{+}=-0.813 \pm 0.145$ (Batley et al. 2011a). The resulting experimental branching ratios are

$$
\begin{aligned}
\operatorname{BR}\left(K^{ \pm} \rightarrow \pi^{ \pm} e^{+} e^{-}\right) & =(3.14 \pm 0.10) \times 10^{-7}, \\
\operatorname{BR}\left(K^{ \pm} \rightarrow \pi^{ \pm} \mu^{+} \mu^{-}\right) & =(9.62 \pm 0.25) \times 10^{-8} .
\end{aligned}
$$

In Figs. 18 and 19 we show the spectra of $K^{+} \rightarrow \pi^{+} \ell^{+} \ell^{-}$ $(\ell=e, \mu)$ and the comparison with the theoretical predictions for the chiral form factors $(6.76)$ and for a linear form factor [without $V_{+}^{\pi \pi}(z)$ in Eq. [6.76]].

For the $K_{S}$ decay only branching ratios are available. By using the ratio $b_{i} / a_{i}=1 / r_{V}^{2}$ given by vector meson

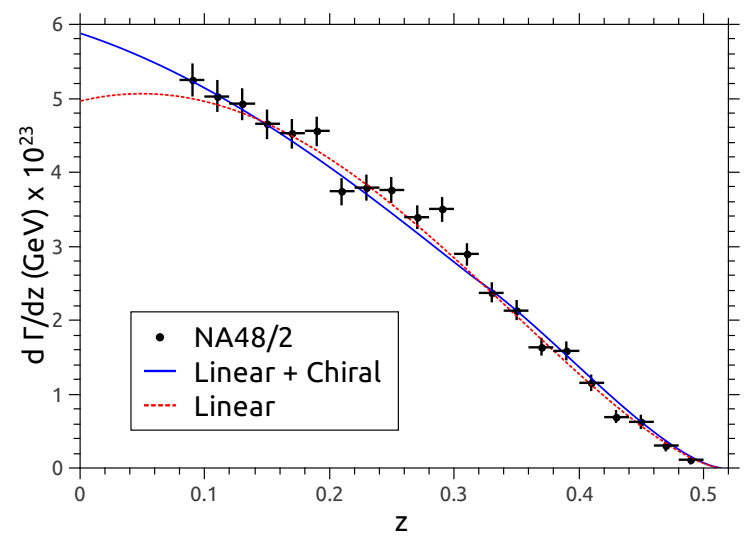

FIG. 18 Spectra for $K^{+} \rightarrow \pi^{+} e^{+} e^{-}$in the dilepton invariant mass. The data are from Batley et al. (2009b).

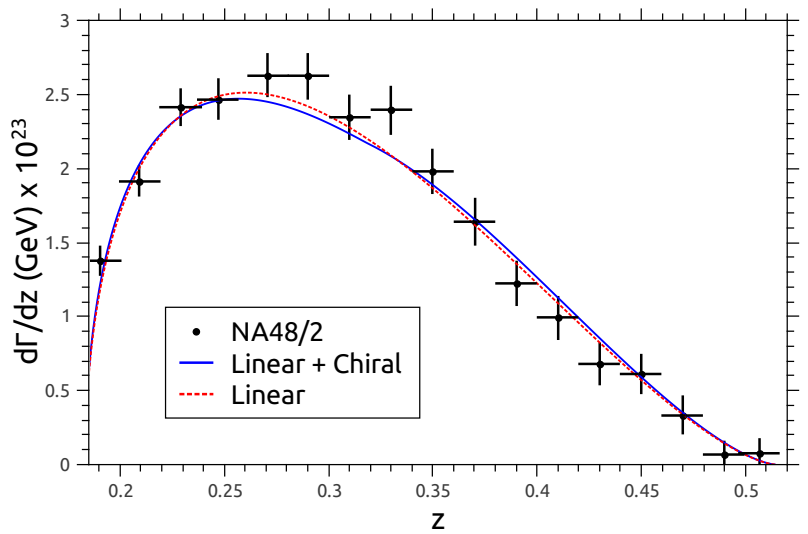

FIG. 19 Spectra for $K^{+} \rightarrow \pi^{+} \mu^{+} \mu^{-}$in the dilepton invariant mass. The data are from Batley et al. (2011a).

dominance (D'Ambrosio et al. 1998a), it is found that $\left|a_{S}\right|=1.06_{-0.21}^{+0.20}$ for the electron (Batley et al., 2003$)$ and $\left|a_{S}\right|=1.54_{-0.32}^{+0.40}$ for the muon case (Batley et al. $2004 \mathrm{~b}$ ). Notice though that the vector meson dominance ratio $b_{i} / a_{i}$ quoted above fails in the charged kaon decay. The measured rates are

$$
\begin{aligned}
\operatorname{BR}\left(K_{S} \rightarrow \pi^{0} e^{+} e^{-}\right) & =\left(5.8_{-2.4}^{+2.9}\right) \times 10^{-9}, \\
\operatorname{BR}\left(K_{S} \rightarrow \pi^{0} \mu^{+} \mu^{-}\right) & =\left(2.9_{-1.2}^{+1.5}\right) \times 10^{-9},
\end{aligned}
$$

where the large errors reflect the low statistics.

Other parametrizations going beyond $\mathcal{O}\left(p^{4}\right)$ have also been put forward, focusing on the polynomial part of $V(z)$. Friot et al. (2004b) assume a minimal narrowresonance structure, while a related vector meson dominance approach is introduced in Dubničková et al. (2008). Unfortunately, the size of present data samples is insufficient to distinguish between models.

In addition to the dominant $K \rightarrow \pi \gamma^{*}$ amplitude, the form factor $V(z)$ receives a short-distance contribution from the operator $Q_{7 V}$ in Eq. (2.9). Although negligible in the branching ratios and spectra, its interfer- 


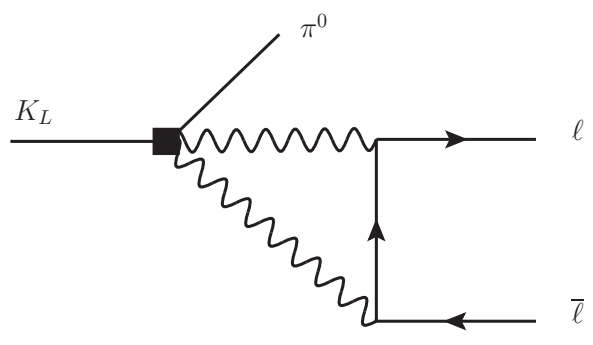

FIG. 20 CP-conserving contribution to $K_{L} \rightarrow \pi^{0} \ell^{+} \ell^{-}$.

ence with the long-distance amplitude leads to a CPviolating charge asymmetry in $K^{ \pm} \rightarrow \pi^{ \pm} \ell^{+} \ell^{-}$(Ecker et al. . 1988). This asymmetry is tiny within the SM (D'Ambrosio et al. 1998a, D'Ambrosio and Gao, 2002).

2. $K_{L} \rightarrow \pi^{0} \ell^{+} \ell^{-}$

$K_{L} \rightarrow \pi^{0}\left(\ell^{+} \ell^{-}\right)_{J=1}$ with the lepton pair in a vector or axial-vector state is CP violating and, accordingly, also $K_{L} \rightarrow \pi^{0} \gamma^{*}$. There are three main contributions to the $K_{L} \rightarrow \pi^{0} \ell^{+} \ell^{-}$decay that, in principle, could be of the same order of magnitude (Donoghue and Gabbiani, 1995; Ecker et al., 1988):

i. A direct CP-violating transition induced by the short-distance Lagrangian 2.5. The relevant contributions come from the operators $Q_{7 V}$ and $Q_{7 A}$ in Eq. 2.9 and are proportional to $\operatorname{Im} \lambda_{t}$.

ii. An indirect $\mathrm{CP}$-violating amplitude due to the $K^{0}-\overline{K^{0}}$ oscillation, proportional to the CPviolating parameter $\epsilon$ :

$$
\left.A\left(K_{L} \rightarrow \pi^{0} \ell^{+} \ell^{-}\right)\right|_{\mathrm{CPV}-\mathrm{ind}}=\epsilon A\left(K_{S} \rightarrow \pi^{0} \ell^{+} \ell^{-}\right) .
$$

iii. A CP-conserving contribution from $K_{L} \rightarrow \pi^{0} \gamma \gamma$ through $\gamma \gamma \rightarrow \ell^{+} \ell^{-}$rescattering, as indicated in Fig. 20.

Let us first consider the CP-violating contributions (Buchalla et al. 2003; D'Ambrosio et al. 1998a, Flynn and Randall, 1989b; Heiliger and Sehgal, 1993a, Isidori et al., 2004). Together with a vector contribution $V(z)$, as in Eq. 6.70), from indirect CP violation and the $Q_{7 V}$ amplitude, the $Q_{7 A}$ operator generates axial-vector and pseudoscalar terms:

$$
\begin{aligned}
V^{\mathrm{ind}}(z) & \simeq \pm \epsilon\left[a_{S}+b_{S} z\right] \\
V^{\mathrm{dir}}(z) & =i \frac{4 \pi y_{7 V}}{\sqrt{2} \alpha} \operatorname{Im} \lambda_{t} f_{+}^{K \pi}(z), \\
A(z) & =i \frac{4 \pi y_{7 A}}{\sqrt{2} \alpha} \operatorname{Im} \lambda_{t} f_{+}^{K \pi}(z), \\
P(z) & =-i \frac{8 \pi y_{7 A}}{\sqrt{2} \alpha} \operatorname{Im} \lambda_{t} f_{-}^{K \pi}(z),
\end{aligned}
$$

where $f_{ \pm}^{K \pi}(z)$ are the $K_{\ell 3}$ form factors. In $V^{\text {ind }}(z)$ we have neglected the tiny unitarity corrections from $K_{S} \rightarrow \pi^{+} \pi^{-} \pi^{0}$ and have made explicit a possible ambiguity in the relative sign with respect to the shortdistance contributions.

The pseudoscalar amplitude is helicity suppressed and can be neglected in the electron case. Using $b_{S} / a_{S}=$ $1 / r_{V}^{2}$, the total CP-violating contribution to the rate is (Buchalla et al., 2003; Buras et al., 1994a)

$$
\begin{aligned}
& \operatorname{BR}\left(K_{L} \rightarrow \pi^{0} e^{+} e^{-}\right)_{\mathrm{CPV}}= \\
& 10^{-12} \times\left[15.7\left|a_{S}\right|^{2} \pm 6.2\left|a_{S}\right|\left(\frac{\operatorname{Im} \lambda_{t}}{10^{-4}}\right)+2.4\left(\frac{\operatorname{Im} \lambda_{t}}{10^{-4}}\right)^{2}\right]
\end{aligned}
$$

For the muon case (including the dependence on $m_{\mu}$ ), one obtains (Isidori et al. 2004 )

$$
\begin{aligned}
& \operatorname{BR}\left(K_{L} \rightarrow \pi^{0} \mu^{+} \mu^{-}\right)_{\mathrm{CPV}}= \\
& 10^{-12} \times\left[3.7\left|a_{S}\right|^{2} \pm 1.6\left|a_{S}\right|\left(\frac{\operatorname{Im} \lambda_{t}}{10^{-4}}\right)+1.0\left(\frac{\operatorname{Im} \lambda_{t}}{10^{-4}}\right)^{2}\right]
\end{aligned}
$$

In both cases the error in the numerical coefficients is estimated to be $10 \%-20 \%$. For an expected value of $a_{S} \sim 1$, the largest contribution comes from the indirect $\mathrm{CP}$-violating component, particularly in the case $\ell=e$. Based on several assumptions on the matching between short- and long-distance dynamics, Buchalla et al. (2003) concluded that a positive interference between the indirect and direct $\mathrm{CP}$-violating components is the most natural setting. Using the values of $\left|a_{S}\right|$ extracted from the analyses of $K_{S} \rightarrow \pi^{0} \ell^{+} \ell^{-}$and considering a positive interference, one finds

$$
\begin{aligned}
\operatorname{BR}\left(K_{L} \rightarrow \pi^{0} e^{+} e^{-}\right)_{\mathrm{CPV}} & =(3.1 \pm 0.9) \times 10^{-11} \\
\mathrm{BR}\left(K_{L} \rightarrow \pi^{0} \mu^{+} \mu^{-}\right)_{\mathrm{CPV}} & =(1.4 \pm 0.5) \times 10^{-11}
\end{aligned}
$$

Now we consider the CP-conserving contribution from $K_{L} \rightarrow \pi^{0} \gamma \gamma$. Neglecting the $y$ dependence of the $2 \gamma$ amplitudes $A, B$ in Eq. 6.54, the combination $A+B$ gives $|\gamma \gamma\rangle_{J=0}$ and the amplitude $B$ provides $|\gamma \gamma\rangle_{J=2}$. However, the transition to $\left|\ell^{+} \ell^{-}\right\rangle$is helicity suppressed $\left(\propto m_{\ell}\right)$ for the initial $J=0$ state (Donoghue et al. 1987). Accordingly, for $\ell=e$ only the amplitude $B$ arising beyond $\mathcal{O}\left(p^{4}\right)$ in the chiral counting] could give a non-negligible contribution, while for $\ell=\mu$ both amplitudes can be significant (Ecker et al., 1988, Flynn and Randall, 1989a; Isidori et al. 2004; Morozumi and Iwasaki, 1989).

Through a naive dimensional analysis of the amplitude $B(z)$, Ecker et al. (1988) concluded that the CPconserving contribution to $K_{L} \rightarrow \pi^{0} e^{+} e^{-}$was much smaller than the $\mathrm{CP}$-violating one, making this decay a good testing ground of the SM. This was later confirmed by a two-loop calculation, including unitarity corrections from $K_{L} \rightarrow \pi^{+} \pi^{-} \pi^{0}$ and ignoring $\mathcal{O}\left(p^{6}\right)$ local contributions (Isidori et al. , 2004), which found values below $10^{-12}$ for the CP-conserving $K_{L} \rightarrow \pi^{0} e^{+} e^{-}$rate. A local contribution to $B(z)$, parametrized through the coupling 
$a_{V}^{0}$ [see Eq. 6.59] ] determined from $K_{L} \rightarrow \pi^{0} \gamma \gamma$ data, results in smaller values around $10^{-13}$. The contribution from $B(z)$ turns out to be very small because of phase space and angular momentum suppression. Therefore, the $K_{L} \rightarrow \pi^{0} e^{+} e^{-}$decay is dominantly $\mathrm{CP}$ violating.

The CP-conserving contribution to the muon channel,

$$
\operatorname{BR}\left(K_{L} \rightarrow \pi^{0} \mu^{+} \mu^{-}\right)_{\mathrm{CPC}}=(5.2 \pm 1.6) \times 10^{-12},
$$

is of the same order but slightly smaller than the CPviolating component (6.86). The interference between $\mathrm{CP}$-conserving and $\mathrm{CP}$-violating amplitudes can then generate a sizable transverse polarization of the muons that could be within reach of the next generation of experiments (Ecker et al. 1988).

The experimental upper bounds on these decays are getting close to the SM predictions. At 90\% C.L., we have (Alavi-Harati et al. 2004)

$$
\operatorname{BR}\left(K_{L} \rightarrow \pi^{0} e^{+} e^{-}\right)<2.8 \times 10^{-10}
$$

and (Alavi-Harati et al., 2000b)

$$
\operatorname{BR}\left(K_{L} \rightarrow \pi^{0} \mu^{+} \mu^{-}\right)<3.8 \times 10^{-10} .
$$

F. $K \rightarrow \pi \pi \gamma^{(*)}$

The amplitude for $K\left(p_{K}\right) \rightarrow \pi_{1}\left(p_{1}\right) \pi_{2}\left(p_{2}\right) \gamma(q)$

$$
A(K \rightarrow \pi \pi \gamma)=\varepsilon_{\mu}^{*}(q) M^{\mu}\left(q, p_{1}, p_{2}\right)
$$

is decomposed into dimensionless electric $(E)$ and magnetic $(M)$ components defined by

$$
M_{\mu}=\frac{E\left(z_{i}\right)}{M_{K}}\left[z_{1} p_{2 \mu}-z_{2} p_{1 \mu}\right]+\frac{M\left(z_{i}\right)}{M_{K}^{3}} \varepsilon_{\mu \nu \rho \sigma} p_{1}^{\nu} p_{2}^{\rho} q^{\sigma},
$$

with $z_{i}=q \cdot p_{i} / M_{K}^{2}(i=1,2)$ and $z_{3}=z_{1}+z_{2}=E_{\gamma}^{*} / M_{K}$, where $E_{\gamma}^{*}$ is the photon energy in the kaon rest frame. Summing over photon helicities, there is no interference between both amplitudes and the double differential rate for an unpolarized photon is given by

$$
\begin{aligned}
& \frac{d^{2} \Gamma}{d z_{1} d z_{2}}=\frac{M_{K}}{(4 \pi)^{3}}\left(\left|E\left(z_{i}\right)\right|^{2}+\left|M\left(z_{i}\right)\right|^{2}\right) \\
& \quad \times\left[z_{1} z_{2}\left(1-2\left(z_{1}+z_{2}\right)-r_{1}^{2}-r_{2}^{2}\right)-r_{1}^{2} z_{2}^{2}-r_{2}^{2} z_{1}^{2}\right],
\end{aligned}
$$

with $r_{i}=M_{\pi_{i}} / M_{K}$. The electric amplitude can be decomposed as the sum of inner-bremsstrahlung (IB) and direct-emission (DE) components, $E=E_{\mathrm{IB}}+E_{\mathrm{DE}}$, while the magnetic amplitude contains DE only. $E_{\mathrm{IB}}$ is determined by the Low theorem (Low, 1958):

$$
E_{\mathrm{IB}}\left(z_{i}\right)=\frac{e}{M_{K} z_{1} z_{2}} A\left(K \rightarrow \pi_{1} \pi_{2}\right) .
$$

In general, $E_{\mathrm{IB}}$ dominates the photon spectrum for low values of the photon energy (in the kaon rest frame), but it is obviously absent for $K_{S}, K_{L} \rightarrow \pi^{0} \pi^{0} \gamma$.
The DE amplitudes explore the electromagnetic structure of the hadron interaction. They are customarily decomposed in a multipole expansion (D'Ambrosio et al. 1992):

$$
\begin{aligned}
E_{\mathrm{DE}} & =E_{1}\left(z_{3}\right)+E_{2}\left(z_{3}\right) \cdot z_{12}+\mathcal{O}\left(z_{12}^{2}\right), \\
M & =M_{1}\left(z_{3}\right)+M_{2}\left(z_{3}\right) \cdot z_{12}+\mathcal{O}\left(z_{12}^{2}\right),
\end{aligned}
$$

with $z_{12}=z_{1}-z_{2}$. This decomposition is particularly relevant for initial states with definite $\mathrm{CP}$ (neutral channels), as even electric and odd magnetic multipoles are $\mathrm{CP}$ odd while the others are $\mathrm{CP}$ even. Hence CP invariance would require the amplitudes $E_{1}, M_{2}, E_{3}, \ldots$ to vanish for $K_{L} \rightarrow \pi^{+} \pi^{-} \gamma$, whereas $M_{1}, E_{2}, M_{3}, \ldots$ vanish for $K_{S} \rightarrow \pi^{+} \pi^{-} \gamma$. For $K^{ \pm} \rightarrow \pi^{ \pm} \pi^{0} \gamma$ all of them are allowed. Moreover, the limited phase space $\left(\left|z_{12}\right| \lesssim 0.17\right)$ indicates that higher-order multipoles are suppressed.

The decays $K \rightarrow \pi\left(p_{1}\right) \pi\left(p_{2}\right) \ell^{+}\left(k_{+}\right) \ell^{-}\left(k_{-}\right)$where the lepton pair is produced by an off-shell photon have amplitudes

$$
A\left(K \rightarrow \pi \pi \ell^{+} \ell^{-}\right)=\frac{e}{q^{2}} V_{\mu} \bar{u}_{\ell}\left(k_{-}\right) \gamma^{\mu} v_{\ell}\left(k_{+}\right),
$$

where $q=k_{+}+k_{-}$and

$$
V_{\mu}=\frac{\mathcal{E}_{1}\left(z_{i}\right)}{M_{K}} p_{1 \mu}+\frac{\mathcal{E}_{2}\left(z_{i}\right)}{M_{K}} p_{2 \mu}+\frac{M\left(z_{i}\right)}{M_{K}^{3}} \varepsilon_{\mu \nu \rho \sigma} p_{1}^{\nu} p_{2}^{\rho} q^{\sigma} .
$$

1. $K^{+} \rightarrow \pi^{+} \pi^{0} \gamma$

The IB contribution to this decay is suppressed by the $\Delta I=1 / 2$ rule because it is proportional to the $K^{+} \rightarrow$ $\pi^{+} \pi^{0}$ amplitude, a $\Delta I=3 / 2$ transition. However, this is still the largest contribution to the rate. The dominant DE contribution is $\mathcal{O}\left(p^{4}\right)$ in CHPT (Bijnens et al. 1992 Ecker et al. 1992). At this order, the electric amplitude has a finite one-loop contribution of which the octet part turns out to be tiny (D'Ambrosio and Isidori, 1995; Ecker et al. 1994):

$$
E_{\text {loop }}^{(4)}=-i e \frac{G_{8} M_{K}}{8 \pi^{2} F_{0}}\left(M_{K}^{2}-M_{\pi}^{2}\right)\left[h_{\pi K}\left(z_{+}\right)+h_{K \eta}\left(z_{+}\right)\right],
$$

where the functions $h_{i j}(z)$ are given in Eq. A4). A sizeable 27-plet contribution to the DE electric amplitude, from an enhanced two-pion loop, has been recently identified (Mertens and Smith, 2011). There is also a local scale-independent contribution

$$
E_{\mathrm{ct}}^{(4)}=2 i e \frac{G_{8} M_{K}^{3}}{F_{0}}\left(N_{14}-N_{15}-N_{16}-N_{17}\right)
$$

in terms of the LECs in Eq. 2.16). Model-dependent estimates (D'Ambrosio and Portolés, 1998b, Ecker et al. 1993) indicate that the vector meson contributions to $E_{\mathrm{ct}}^{(4)}$ are suppressed whereas an axial-vector meson exchange piece remains. This amplitude could be measured from the interference with the IB contribution. The 
$\mathcal{O}\left(p^{4}\right)$ magnetic amplitude has a reducible component from the Wess-Zumino-Witten anomalous Lagrangian in Eq. 2.15) and a direct non-anomalous contribution from odd-intrinsic-parity operators in Eq. (2.16):

$$
M^{(4)}=-\frac{e}{2 \pi^{2}} \frac{G_{8} M_{K}^{3}}{F_{0}}\left[1-16 \pi^{2}\left(3 N_{29}-N_{30}\right)\right] .
$$

A factorization estimate of the couplings (D'Ambrosio and Portolés, 1998b indicates that the different contributions are of the same order and interfere constructively. Some $\mathcal{O}\left(p^{6}\right)$ analyses were also performed (D'Ambrosio and Isidori, 1995; Ecker et al., 1994) but the lack of knowledge on the LECs at this order makes it difficult to assess their relevance. The role of form factors induced by vector meson exchange was also explored (Cappiello and D'Ambrosio, 2007, D'Ambrosio and Gao, 2000).

The differential cross section is usually written in terms of $T_{C}^{*}$, the kinetic energy of the charged pion in the kaon rest frame, and of $W^{2}=\left(q \cdot p_{K}\right)\left(q \cdot p_{+}\right) /\left(M_{\pi^{+}}^{2} M_{K}^{2}\right)$, factorizing the IB contribution (D'Ambrosio et al. 1992),

$$
\begin{array}{r}
\frac{d^{2} \Gamma^{ \pm}}{d T_{c}^{*} d W^{2}}=\frac{d^{2} \Gamma_{I B}^{ \pm}}{d T_{c}^{*} d W^{2}}\left[1+2 \cos \left(\delta_{1}^{1}-\delta_{0}^{2} \pm \phi\right) Y_{E} W^{2}\right. \\
\left.+\left(Y_{E}^{2}+Y_{M}^{2}\right) W^{4}\right],
\end{array}
$$

where $\Gamma^{+}\left(\Gamma^{-}\right)$corresponds to the decay of $K^{+}\left(K^{-}\right)$. Here $\phi$ is a possible CP-violating phase, $\delta_{\ell}^{I}$ are the strong rescattering phases for a final $\pi \pi$ state of isospin $I$ and orbital angular momentum $\ell . Y_{E}, Y_{M}$ are the DE electric and magnetic amplitudes, respectively, normalized to $K^{ \pm} \rightarrow \pi^{ \pm} \pi^{0}$. It is usually assumed that $Y_{E}$ and $Y_{M}$ are nearly independent of $T_{c}^{*}$ and are therefore constants over the Dalitz plot. The linear term in $W^{2}$ corresponds to the interference between the amplitudes $E_{\mathrm{DE}}$ and $E_{\mathrm{IB}}$. A recent measurement by NA48/2 (Batley et al., 2010b) establishes that $Y_{M} / Y_{E}=-11 \pm 3$, showing that among the $\mathrm{DE}$ amplitudes the magnetic contribution dominates.

Charge asymmetries in this channel are interesting observables for CP-violating effects (D'Ambrosio and Isidori, 1998). A bound on the fully integrated asymmetry $\delta \Gamma=\left(\Gamma^{+}-\Gamma^{-}\right) /\left(\Gamma^{+}+\Gamma^{-}\right)$has recently been published (Batley et al. $2010 \mathrm{~b}):|\delta \Gamma|<1.5 \times 10^{-3}$ at $90 \%$ C.L. translates into $|\sin \phi|<0.56$. The asymmetry constructed with the partially integrated decay widths (Colangelo et al. 1999) is also of interest:

$$
\frac{d A_{W}}{d W^{2}}=\frac{d \Gamma^{+} / d W^{2}-d \Gamma^{-} / d W^{2}}{d \Gamma^{+} / d W^{2}+d \Gamma^{-} / d W^{2}} .
$$

Batley et al. (2010b) obtain $A_{W}=(-0.6 \pm 1.0) \times 10^{-3}$.

\section{2. $K_{L} \rightarrow \pi^{+} \pi^{-} \gamma$}

The IB component is proportional to the amplitude for $K_{L} \rightarrow \pi^{+} \pi^{-}$. Consequently, it violates $\mathrm{CP}$ and it is rather small. Assuming CP invariance, the dominant contribution is given by the $M_{1}$ amplitude because $E_{2}$ is

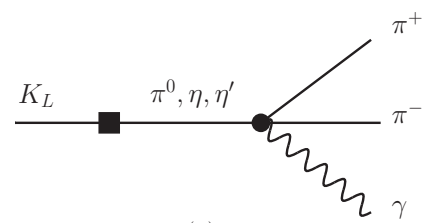

(a)

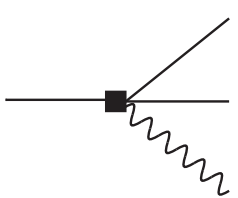

(b)
FIG. 21 Diagram (a) has anomalous and resonancedominated contributions to $K_{L} \rightarrow \pi^{+} \pi^{-} \gamma$. Diagram (b) gives a direct weak transition in terms of the couplings in Eq. 2.16.

suppressed by phase space. These features make this decay very suitable to study the DE magnetic amplitude (D'Ambrosio and Gao, 2000, D'Ambrosio and Isidori, 1995 D'Ambrosio and Portolés, 1998a; Ecker et al. 1992, 1994 Lin and Valencia, 1988 Picciotto 1992).

The leading $E_{2}$ amplitude arises at $\mathcal{O}\left(p^{4}\right)$ in CHPT. There is no local contribution and therefore $E_{2}$ is given by a finite one-loop result (D'Ambrosio and Isidori, 1995 Ecker et al., 1994):

$$
\begin{aligned}
E_{\text {loop }}^{(4)}= & i e \frac{G_{8} M_{K}}{8 \pi^{2} F_{0}}\left(M_{K}^{2}-M_{\pi}^{2}\right)\left[h_{\pi K}\left(z_{-}\right)+h_{K \eta}\left(z_{-}\right)\right. \\
& \left.-h_{\pi K}\left(z_{+}\right)-h_{K \eta}\left(z_{+}\right)\right],
\end{aligned}
$$

with $h_{i j}(z)$ given in Eq. A4). However, as commented above, this contribution is even smaller than the CPviolating IB component: $\left|E_{\text {loop }}^{(4)} / E_{\mathrm{IB}}\right| \sim 10^{-2}$. The 27-plet contribution to the $E_{2}$ amplitude has also been determined (Mertens and Smith, 2011).

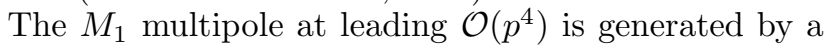
constant local contribution (Ecker et al. 1994$)$ in terms of the LECs in Eq. 2.16):

$$
M^{(4)}=-16 e \frac{G_{8} M_{K}^{3}}{F_{0}}\left(N_{29}+N_{31}\right) .
$$

The magnetic amplitude was also studied at $\mathcal{O}\left(p^{6}\right)$, including both local (D'Ambrosio and Portolés, 1998a; Ecker et al. 1994, Lin and Valencia, 1988) and one-loop contributions (D'Ambrosio and Portolés, 1998a). A piece of the local term is completely fixed by the anomalous WZW Lagrangian in Eq. 2.15, as shown in Fig. 21]:

$$
M_{\text {anom }}^{(6)}=e \frac{G_{8} M_{K}^{3}}{2 \pi^{2} F_{0}} F_{1}(\hat{\rho}, \xi, \theta),
$$

in terms of $\theta, \xi$ and $\hat{\rho}$ defined in Eqs. 6.20, 6.21) and 6.22 , where

$$
\begin{aligned}
& F_{1}=\frac{1}{1-r_{\pi}^{2}}+ \frac{1}{3\left(1-r_{\eta}^{2}\right)}[(1+\xi) \cos \theta+2 \sqrt{2} \hat{\rho} \sin \theta] \\
& \times\left[\left(\frac{F_{\pi}}{F_{\eta_{8}}}\right)^{3} \cos \theta-\sqrt{2}\left(\frac{F_{\pi}}{F_{\eta_{0}}}\right)^{3} \sin \theta\right] \\
&- \frac{1}{3\left(1-r_{\eta^{\prime}}^{2}\right)}[2 \sqrt{2} \hat{\rho} \cos \theta-(1+\xi) \sin \theta] \\
& \times\left[\left(\frac{F_{\pi}}{F_{\eta_{8}}}\right)^{3} \sin \theta+\sqrt{2}\left(\frac{F_{\pi}}{F_{\eta_{0}}}\right)^{3} \cos \theta\right] \cdot
\end{aligned}
$$


This expression includes higher orders beyond $\mathcal{O}\left(p^{6}\right)$.

The first $z_{3}$ dependence comes with resonance dominated local counterterms that depend both on strong vertices (with a weak transition in the external kaon leg) and pure weak vertices (D'Ambrosio and Portolés, 1998a). A similar situation arises in the one-loop amplitude $M_{\text {loop }}^{(6)}=M_{\mathrm{WZW}}+M_{N_{i}}$ where the first term originates from the WZW Lagrangian with a weak transition in the external kaon leg, and the second is determined by vertices of the weak Lagrangian (2.16). Unfortunately, due to the presence of weak LECs, it is not possible to provide a model-independent theoretical prediction.

The phenomenological analysis was originally carried out with only the magnetic amplitude and with a $\rho$-pole dominated form factor (Lin and Valencia, 1988):

$$
M=e \frac{G_{8} M_{K}^{3}}{2 \pi^{2} F_{0}} \tilde{g}_{M_{1}}\left(1+\frac{a_{1} / a_{2}}{\left(M_{\rho}^{2}-M_{K}^{2}\right)+2 M_{K}^{2} z_{3}}\right) .
$$

Both parameters were determined by Abouzaid et al. (2006b): $\left|\tilde{g}_{M_{1}}\right|=1.20 \pm 0.09$ and $a_{1} / a_{2}=(-0.738 \pm$ $0.019) \mathrm{GeV}^{2}$. A model-independent parametrization is provided by a power series expansion in the variable $z_{3}$ :

$$
M=e \frac{G_{8} M_{K}^{3}}{2 \pi^{2} F_{0}} g_{M_{1}}\left(1+c z_{3}\right) .
$$

The values obtained from the expression 6.106 correspond to $\left|g_{M_{1}}\right|=1.30 \pm 0.12$ and $c=-1.74 \pm 0.08$. It was noticed (Ecker et al. , 1994) that the slope is rather large compared with naive expectations based on resonance saturation. Indeed, a quadratic approximation also shows rather large values for both linear and quadratic slopes (Alavi-Harati et al. 2001e). Although the value of $c$ can be easily accommodated for a reasonable range of the parameters involved in the theoretical analyses (D'Ambrosio and Portolés, 1998a : Ecker et al., 1994), the predicted photon spectrum is slightly at variance with the experimental determination. The ratio between the width due to the DE magnetic amplitude and the total width was also measured: $\Gamma_{\mathrm{DE}} /\left(\Gamma_{\mathrm{DE}}+\Gamma_{\mathrm{IB}}\right)=$ $0.689 \pm 0.021$ for $E_{\gamma} \geq 20 \mathrm{MeV}$ (Abouzaid et al. 2006b). The full branching ratio is

$$
\operatorname{BR}\left(K_{L} \rightarrow \pi^{+} \pi^{-} \gamma\right)=(4.15 \pm 0.15) \times 10^{-5} .
$$

Both IB and $E_{1}$ amplitudes are $\mathrm{CP}$ violating. It is usual to parametrize this violation through

$$
\begin{aligned}
\left|\eta_{+-\gamma}\right| \mathrm{e}^{i \phi_{+-\gamma}} & =\frac{\left.A\left(K_{L} \rightarrow \pi^{+} \pi^{-} \gamma\right)\right|_{\mathrm{IB}+E_{1}}}{A\left(K_{S} \rightarrow \pi^{+} \pi^{-} \gamma\right)}, \\
\epsilon_{+-\gamma}^{\prime} & =\eta_{+-\gamma}-\eta_{+-},
\end{aligned}
$$

where $\eta_{+-}$is defined in Eq. (5.13). The experimental determination of these quantities is already rather old (Matthews et al., 1995. Ramberg et al. 1993): $\left|\eta_{+-\gamma}\right|=(2.36 \pm 0.06) \times 10^{-3}, \phi_{+-\gamma}=(44 \pm 0.4)^{\circ}$ and $\left|\epsilon_{+-\gamma}^{\prime}\right| / \epsilon<0.3$ at $90 \%$ C.L., in accordance with SM predictions (Tandean and Valencia, 2000). More recent is a measurement of the equivalent of the parameter $g_{M_{1}}$ in Eq. 6.107) for the amplitude $E_{1}$ : Abouzaid et al. (2006b) find $\left|g_{E_{1}}\right| \leq 0.21$ (90\% C.L.).

\section{3. $K_{S} \rightarrow \pi^{+} \pi^{-} \gamma$}

Contrary to the $K_{L}$ decay, this channel is dominated by the IB amplitude because there is no suppression in the $K_{S} \rightarrow \pi^{+} \pi^{-}$decay. From the remaining contributions, $M_{1}$ and $E_{2}$ are CP violating. Therefore, the dominant DE amplitude is $E_{1}$ with a leading contribution of $\mathcal{O}\left(p^{4}\right)$. The octet piece (D'Ambrosio and Isidori, 1995 D'Ambrosio et al., 1993; Mertens and Smith, 2011) contains a scale-independent local amplitude

$$
E_{\mathrm{ct}}^{(4)}=4 i e \frac{G_{8} M_{K}^{3}}{F_{0}}\left[N_{14}-N_{15}-N_{16}-N_{17}\right],
$$

with the same combination of LECs that appears in $K^{ \pm} \rightarrow \pi^{ \pm} \pi^{0} \gamma$ in Eq. (6.98). In addition, there is a finite one-loop contribution

$$
\begin{aligned}
E_{\text {loop }}^{(4)}= & -i e \frac{G_{8} M_{K}}{8 \pi^{2} F_{0}}\left(M_{K}^{2}-M_{\pi}^{2}\right)\left[-4 h_{\pi \pi}\left(-z_{3}\right)\right. \\
& \left.+h_{\pi K}\left(z_{+}\right)+h_{K \eta}\left(z_{+}\right)+\left(z_{+} \leftrightarrow z_{-}\right)\right] .
\end{aligned}
$$

The 27-plet amplitude has been determined by Mertens and Smith (2011). Depending on the unknown combination of LECs in Eq. 6.110), the ratio of IB and DE contributions amounts to $\Gamma_{\mathrm{IB}} / \Gamma_{\mathrm{DE}} \sim 10^{3}$ and IB therefore dominates the experimental branching ratio (Nakamura et al. 2010)

$\operatorname{BR}\left(K_{S} \rightarrow \pi^{+} \pi^{-} \gamma, E_{\gamma}^{*}>50 \mathrm{MeV}\right)=(1.79 \pm 0.05) \times 10^{-3}$.

\section{4. $K \rightarrow \pi \pi \ell^{+} \ell^{-}$}

The lepton pair in this decay is produced by an offshell photon and in consequence the dynamics is that of the $K \rightarrow \pi \pi \gamma^{*}$ process. The widths are smaller than those for a real photon and only the decays $K_{L}, K_{S} \rightarrow \pi^{+} \pi^{-} e^{+} e^{-}$have been observed experimentally (Abouzaid et al., 2006a; Lai et al., 2003). This decay provides a useful tool to look for the CP-violating interference between electric and magnetic amplitudes because the lepton plane furnishes a measurement of the photon polarization vector. Bounds on the decays $K_{L} \rightarrow \pi^{0} \pi^{0} \ell^{+} \ell^{-}$have also been determined for $\ell=e$ (Alavi-Harati et al. 2002 ) and $\ell=\mu$ (Abouzaid et al. 2011b). As the $\mu^{+} \mu^{-}$case is strongly suppressed by the available phase space, we only consider $e^{+} e^{-}$production.

The decay amplitude is given by Eqs. (6.95) and (6.96). A first study of the decay $K_{L} \rightarrow \pi^{+} \pi^{-} e^{+} e^{-}$with constant electric and magnetic amplitudes was carried out by Sehgal and Wanninger (1992), while the calculation at leading $\mathcal{O}\left(p^{4}\right)$ of the electric amplitudes (keeping a constant magnetic contribution) was performed in Elwood 
et al. 1995). The complete results up to $\mathcal{O}\left(p^{4}\right)$ for both $K_{L} \rightarrow \pi^{+} \pi^{-} e^{+} e^{-}$and $K^{+} \rightarrow \pi^{+} \pi^{0} e^{+} e^{-}$can be found in Pichl (2001).

i. $K_{L} \rightarrow \pi^{+} \pi^{-} e^{+} e^{-}$

For this decay $\mathcal{E}_{2}=-\mathcal{E}_{1}\left(p_{1} \leftrightarrow p_{2}\right)$ and we will only quote the amplitude $\mathcal{E}_{1}$. The leading tree-level contribution to the electric amplitudes is provided by $K^{0}-\overline{K^{0}}$ mixing with an off-shell photon radiating off the pion legs. Therefore, it is proportional to the indirect $\mathrm{CP}$-violation parameter $\epsilon$ :

$\mathcal{E}_{1}^{(2)}=-4 i e \epsilon \frac{G_{8} M_{K} F_{0}}{2 M_{K}^{2} z_{1}+q^{2}}\left(M_{K}^{2}-M_{\pi}^{2}\right)$.

At $\mathcal{O}\left(p^{4}\right)$ a local scale-dependent contribution arises,

$\mathcal{E}_{1 \mathrm{ct}}^{(4)}=2 i e \frac{G_{8} M_{K}}{3 F_{0}} q^{2}\left[N_{14}^{r}-N_{15}^{r}-3\left(N_{16}^{r}-N_{17}\right)\right]$,

together with a divergent one-loop amplitude (Pichl, 2001). At this order, the magnetic amplitude is constant and coincides with the case of a real photon in Eq. 6.103). In Pichl (2001) the magnetic amplitude was modified by including a $\rho$-pole parametrization analogous to (6.106).

ii. $K^{+} \rightarrow \pi^{+} \pi^{0} e^{+} e^{-}$

For this channel there is no relation between the two electric amplitudes as in the $K_{L}$ decay. It is interesting to observe that at leading $\mathcal{O}\left(p^{2}\right)$ the octet contribution vanishes in the isospin limit and only the 27-plet representation gives a non-zero contribution (Pichl, 2001). At $\mathcal{O}\left(p^{4}\right)$ there are local scaledependent amplitudes

$$
\begin{aligned}
\mathcal{E}_{1 \mathrm{ct}}^{(4)}= & -i e \frac{G_{8} M_{K}}{3 F_{0}}\left[-6 M_{K}^{2} z_{2}\left(N_{14}-N_{15}-N_{16}-N_{17}\right)\right. \\
& \left.-4 q^{2}\left(N_{14}^{r}-N_{15}^{r}\right)\right], \\
\mathcal{E}_{2 \mathrm{ct}}^{(4)}= & -i e \frac{G_{8} M_{K}}{3 F_{0}}\left[6 M_{K}^{2} z_{2}\left(N_{14}-N_{15}-N_{16}-N_{17}\right)\right. \\
& \left.-2 q^{2}\left(N_{14}^{r}+2 N_{15}^{r}\right)+6 q^{2}\left(N_{16}^{r}-N_{17}\right)\right], \quad(6.115)
\end{aligned}
$$

reducing to the scale-independent result (6.98) for an on-shell photon. There is also a divergent oneloop contribution at $\mathcal{O}\left(p^{4}\right)$. As in the previous case, the magnetic amplitude is constant and coincides with the case of a real photon in Eq. 6.99).

The branching ratios of $K_{L}$ and $K_{S}$ decays were measured by NA48 (Batley et al., 2011b; Lai et al., 2003):

$$
\begin{aligned}
& \operatorname{BR}\left(K_{L} \rightarrow \pi^{+} \pi^{-} e^{+} e^{-}\right)=(3.08 \pm 0.20) \times 10^{-7}, \\
& \operatorname{BR}\left(K_{S} \rightarrow \pi^{+} \pi^{-} e^{+} e^{-}\right)=(4.93 \pm 0.14) \times 10^{-5} .
\end{aligned}
$$

For the $K_{L}$ decay Pichl (2001) studied the impact of the weak LECs appearing in the amplitudes. However, as pointed out before, the main interest of this process is the study of the interference between electric and magnetic amplitudes provided by the measurement of the photon polarization (Ecker and Pichl, 2001; Elwood et al., 1996, Heiliger and Sehgal, 1993c; Sehgal and van Leusen, 1999, 2000 Sehgal and Wanninger, 1992). As we have seen before, the electric amplitude of $K_{L} \rightarrow \pi^{+} \pi^{-} e^{+} e^{-}$is generated by the indirect $\mathrm{CP}$-violating mixing of $K^{0}$ and $\overline{K^{0}}$. Its interference with the $\mathrm{CP}$-conserving magnetic amplitude produces an asymmetry in the distribution of the angle $\phi$ between the $e^{+} e^{-}$and $\pi^{+} \pi^{-}$planes in the kaon center-of-mass system:

$$
\mathcal{A}_{\mathrm{CP}}=\frac{\int_{0}^{\pi / 2} \frac{d \Gamma}{d \phi} d \phi-\int_{\pi / 2}^{\pi} \frac{d \Gamma}{d \phi} d \phi}{\int_{0}^{\pi / 2} \frac{d \Gamma}{d \phi} d \phi+\int_{\pi / 2}^{\pi} \frac{d \Gamma}{d \phi} d \phi} .
$$

This observable was measured ${ }^{7}$ by Abouzaid et al. (2006a) as $\mathcal{A}_{\mathrm{CP}}=(14 \pm 2) \%$. Ecker and Pichl (2001) observed that the theoretical prediction of $\mathcal{A}_{\mathrm{CP}}$ with the leading electric and magnetic amplitudes in Eqs. 6.103) and 6.113 is short by almost a factor two compared with experiment. With the inclusion of an energy dependence in the magnetic amplitude along the model in Eq. 6.106), agreement with the experimental determination is restored.

\section{G. Other decays}

1. $K^{0} \rightarrow \gamma \gamma \gamma$

The CP-preserving amplitude for this process has parity-violating and parity-conserving contributions:

$$
A\left(K^{0} \rightarrow \gamma \gamma \gamma\right)=\varepsilon_{\alpha}^{*}\left(q_{1}\right) \varepsilon_{\beta}^{*}\left(q_{2}\right) \varepsilon_{\gamma}^{*}\left(q_{3}\right)\left(M_{\mathrm{PV}}^{\alpha \beta \gamma}+M_{\mathrm{PC}}^{\alpha \beta \gamma}\right) .
$$

The first contributes to $K_{L} \rightarrow \gamma \gamma \gamma$ while the parityconserving amplitude governs $K_{S} \rightarrow \gamma \gamma \gamma$. The Lorentz structure of both amplitudes is rather complex and can be found in Heiliger et al. (1994) and Ho and Tandean (2010). Both amplitudes are strongly suppressed by angular momentum. This is due to the fact that any two of the three photons in the final state must have $J \geq 2$ as a result of gauge invariance and Bose symmetry.

Estimates of the branching ratios have recently been carried out (Ho and Tandean, 2010) by using naive dimensional analysis to predict the order of magnitude of the LECs in the appropriate chiral Lagrangian of $\mathcal{O}\left(p^{10}\right)$ contributing at leading order:

$$
\begin{aligned}
& 10^{-16} \leq \mathrm{BR}\left(K_{L} \rightarrow \gamma \gamma \gamma\right) \leq 10^{-14} \\
& 10^{-19} \leq \mathrm{BR}\left(K_{S} \rightarrow \gamma \gamma \gamma\right) \leq 10^{-17}
\end{aligned}
$$

\footnotetext{
7 The analogous asymmetry for the $K_{S}$ decay is compatible with zero (Batley et al. 2011b).
} 
There is a recent experimental upper bound on the first process (Tung et al., 2011):

$$
\mathrm{BR}\left(K_{L} \rightarrow \gamma \gamma \gamma\right)<7.4 \times 10^{-8} \quad(90 \% \text { C.L. }) .
$$

2. $K_{L} \rightarrow \gamma \gamma \ell^{+} \ell^{-}$

This decay is relevant in order to determine the background subtraction for $K_{L} \rightarrow \pi^{0} \ell^{+} \ell^{-}$(Greenlee, 1990). Its theoretical description as a bremsstrahlung radiation off the leptonic legs in the decay $K_{L} \rightarrow \gamma \ell^{+} \ell^{-}$appears to be in agreement with the experimental branching ratios (Alavi-Harati et al. 2000a, 2001a):

$$
\begin{aligned}
& \operatorname{BR}\left(K_{L} \rightarrow \gamma \gamma e^{+} e^{-}, E_{\gamma}^{*}>5 \mathrm{MeV}\right)=(5.8 \pm 0.4) \times 10^{-7} \\
& \operatorname{BR}\left(K_{L} \rightarrow \gamma \gamma \mu^{+} \mu^{-}, m_{\gamma \gamma}>1 \mathrm{MeV}\right)=\left(10_{-6}^{+8}\right) \times 10^{-9}
\end{aligned}
$$

\section{3. $K_{L} \rightarrow \gamma \nu \bar{\nu}$}

This decay exhibits an interesting interplay between long- and short-distance contributions. Both of them have been considered (Richardson and Picciotto, 1995): 1) A long-distance amplitude consisting of two pieces, a weak transition in the $K_{L}$ leg followed by constituent quark triangle diagrams, and a resonance-dominated amplitude; 2) A short-distance contribution given by $d \bar{s} \rightarrow$ $\gamma \nu \bar{\nu}$. The results obtained within this approach give

$$
\begin{aligned}
& \left.\operatorname{BR}\left(K_{L} \rightarrow \gamma \nu \bar{\nu}\right)\right|_{\mathrm{ld}} \sim 10^{-12}, \\
& \left.\operatorname{BR}\left(K_{L} \rightarrow \gamma \nu \bar{\nu}\right)\right|_{\mathrm{sd}} \sim 10^{-11},
\end{aligned}
$$

showing that the process is short-distance dominated (Ma and Okada, 1978).

The CP-conserving and CP-violating short-distance contributions were estimated to be (Geng et al. 2000)

$$
\begin{aligned}
& \left.\operatorname{BR}\left(K_{L} \rightarrow \gamma \nu \bar{\nu}\right)\right|_{\mathrm{CPC}}=1.0 \times 10^{-13}, \\
& \left.\operatorname{BR}\left(K_{L} \rightarrow \gamma \nu \bar{\nu}\right)\right|_{\mathrm{CPV}}=1.5 \times 10^{-15},
\end{aligned}
$$

somewhat at odds with the results mentioned above. Given the small size of these branching ratios, this process is a good benchmark to look for effects beyond the SM (Jiang et al., 2003).

\section{4. $K_{S} \rightarrow \ell_{1}^{+} \ell_{1}^{-} \ell_{2}^{+} \ell_{2}^{-}$}

If $\mathrm{CP}$ is conserved, these decays proceed via

$$
K_{1}^{0} \rightarrow \gamma^{*} \gamma^{*} \rightarrow \ell_{1}^{+} \ell_{1}^{-} \ell_{2}^{+} \ell_{2}^{-} .
$$

To leading order, $\mathcal{O}\left(G_{8} p^{4}\right)$, only one-loop diagrams with pions contribute to the amplitudes. The branching ratios were estimated by Birkfellner (1996):

$$
\begin{aligned}
\operatorname{BR}\left(K_{S} \rightarrow e^{+} e^{-} e^{+} e^{-}\right) & =7 \times 10^{-11}, \\
\operatorname{BR}\left(K_{S} \rightarrow e^{+} e^{-} \mu^{+} \mu^{-}\right) & =8 \times 10^{-12}, \\
\operatorname{BR}\left(K_{S} \rightarrow \mu^{+} \mu^{-} \mu^{+} \mu^{-}\right) & =1 \times 10^{-14}
\end{aligned}
$$

No experimental limits are available.
5. $K_{L}, K_{S} \rightarrow \pi^{0} \pi^{0} \gamma$

The absence of charged particles in these decays implies that they do not have an IB contribution. Moreover, in the CP limit the amplitude for the $K_{L}$ decay is purely electric and the one of $K_{S}$ is purely magnetic. In addition, Bose symmetry requires both multipoles to be even. A consequence of all these features is that the branching ratios are very small. They have still not been observed experimentally (Abouzaid et al., 2008c):

$$
\operatorname{BR}\left(K_{L} \rightarrow \pi^{0} \pi^{0} \gamma\right)<2.43 \times 10^{-7} \quad(90 \% \text { C.L. })
$$

For the $K_{L}$ decay the leading multipole amplitude $E_{2}$ is at least of $\mathcal{O}\left(p^{6}\right)$, because the $\mathcal{O}\left(p^{4}\right)$ octet contributions, both local and loop pieces, vanish (Funck and Kambor, 1993). The 27-plet contributions to the $\mathcal{O}\left(p^{4}\right)$ one-loop amplitude give a tiny addition (Mertens and Smith, 2011). A naive chiral dimensional estimate of the $\mathcal{O}\left(p^{6}\right)$ local contributions predicts $\operatorname{BR}\left(K_{L} \rightarrow \pi^{0} \pi^{0} \gamma\right) \simeq$ $10^{-10}$ (D'Ambrosio and Isidori, 1995; Ecker et al. 1994 ; Mertens and Smith, 2011). However, other estimates predict a larger branching ratio $\simeq 10^{-8}$ (Heiliger and Sehgal, $1993 \mathrm{~b})$. The $K_{S}$ decay has not been studied in detail but it is expected to give a much smaller branching ratio (Heiliger and Sehgal, 1993b).

\section{6. $K_{L}, K_{S} \rightarrow \pi^{0} \pi^{0} \gamma \gamma$}

The decay $K_{L} \rightarrow \pi^{0} \pi^{0} \gamma \gamma$ was studied at leading order in CHPT (Dykstra et al., 1991; Funck and Kambor, 1993). It was found to be dominated by the pion-pole contribution, showing a strong dependence on the cut on the photon energies.

The $K_{S}$ decay has no local contribution at $\mathcal{O}\left(p^{4}\right)$. The amplitude at this order is therefore given by a finite oneloop piece (Funck and Kambor, 1993) that provides an unambiguous prediction:

$$
\operatorname{BR}\left(K_{S} \rightarrow \pi^{0} \pi^{0} \gamma \gamma, m_{\gamma \gamma}>20 \mathrm{MeV}\right)=4.7 \times 10^{-9} .
$$

There are no experimental bounds for these decays.

\section{7. $K \rightarrow 3 \pi \gamma$}

The experimental status of $K \rightarrow 3 \pi \gamma$ decays is still rather meager. Only the two channels with a charged kaon have been detected experimentally. None of the decay modes of a neutral kaon have been seen.

Like in many other radiative $K$ decays, there is both an electric and a magnetic amplitude. The magnetic contributions are severely suppressed for all channels (D'Ambrosio et al. 1997), especially for the ones where the non-radiative decay amplitudes are dominantly octet: $K^{+} \rightarrow \pi^{0} \pi^{0} \pi^{+} \gamma, K^{+} \rightarrow \pi^{+} \pi^{+} \pi^{-} \gamma$ and $K_{L} \rightarrow$ $\pi^{+} \pi^{-} \pi^{0} \gamma$. For these channels, the rates can be predicted with great accuracy by using the concept of "generalized bremsstrahlung" (D'Ambrosio et al. 1996). In this 
way, optimal use can be made of available experimental information on the non-radiative amplitudes. However, as long as the minimal photon energy $E_{\gamma}^{\min }$ is small $\left(E_{\gamma}^{\min } \lesssim 40 \mathrm{MeV}\right)$, the difference between "generalized bremsstrahlung" and the leading Low contribution (Low, 1958) will not be accessible experimentally in the foreseeable future. The genuinely radiative contributions (direct emission) would only matter for large $E_{\gamma}^{\mathrm{min}}$, of course at the expense of the number of events.

The situation is different for the transition $K_{S} \rightarrow$ $\pi^{+} \pi^{-} \pi^{0} \gamma$. At lowest chiral order, the amplitude can only proceed through a $\Delta I=3 / 2$ transition (via bremsstrahlung) and it is therefore suppressed by the $\Delta I=1 / 2$ rule. Similarly as for $K^{+} \rightarrow \pi^{+} \pi^{0} \gamma$, the NLO contributions generated by octet operators become relatively more important. The electric amplitude is dominated by NLO weak LECs, with a large theoretical uncertainty (D'Ambrosio et al., 1997).

The comparison between theory and experiment is displayed in Table VII. For the two charged $K$ decays and for $K_{L} \rightarrow \pi^{+} \pi^{-} \pi^{0} \gamma$, the theoretical accuracy is only limited by the precision with which the parameters of the non-radiative amplitudes are known. The branching ratio for $K_{S} \rightarrow \pi^{+} \pi^{-} \pi^{0} \gamma$ has a much larger theoretical uncertainty. Experimental information on this mode would therefore be especially interesting, but the expected rate is unfortunately very small.

\section{CONCLUSIONS AND OUTLOOK}

Kaons have been at the center of many groundbreaking developments in particle physics, which are worth repeating here. From the introduction of internal "flavor" quantum numbers (strangeness), to parity violation, meson-antimeson mixing, quark mixing, $\mathrm{CP}$ violation, and the suppression of flavor-changing neutral currents, kaon physics has played a key role in the construction of what we now call the Standard Model.

In this review we have summarized the status of theoretical predictions for all SM allowed kaon decays with branching ratios greater than $10^{-11}$. Theoretical predictions rely on the weak operator product expansion to organize the short-distance dynamics, the renormalization group to evolve the relevant operators down to the hadronic scale, and non-perturbative methods such as CHPT and lattice QCD to deal with low-energy strong dynamics. The accuracy of theoretical predictions ranges from excellent to just fair, depending on whether the decay amplitude is dominated by short- or long-distance effects. In all cases we have compared theoretical predictions to existing data and we have discussed the impact of future measurements.

It is remarkable that to date kaons still provide unique information on physics at vastly separated energy scales:
- On one hand, kaons are an excellent probe of lowenergy strong interaction dynamics $\left(E \sim \Lambda_{Q C D}\right)$. While many kaon decays are by themselves a valuable testing ground for chiral effective theory techniques, specific modes such as $K \rightarrow \pi \pi \ell \nu$ and $K \rightarrow \pi \pi \pi$ allow one to extract reliable information on low-energy $\pi \pi$ scattering, thus testing our understanding of purely strong dynamics.

- On the other hand, kaon decays encode information about flavor dynamics at the electroweak scale $E \sim O\left(10^{2-3}\right) \mathrm{GeV}$ through their sensitivity to virtual exchange of heavy SM particles $(W, Z, t)$ and possibly non-standard particles and their flavor structure. Theoretical predictions for $K^{0}-\overline{K^{0}}$ mixing, short-distance dominated rare decays, and semileptonic branching ratios have reached a level of accuracy that already puts very stringent constraints on extensions of the SM, in ways often complementary to other flavor observables and electroweak precision tests. Here we have not discussed in a systematic way the impact of kaon decays on extensions of the SM, which can be found in reviews such as Artuso et al. (2008) and Antonelli et al. (2010b) in the broader context of the lowenergy/high-intensity frontier.

While by now kaon physics is a mature field, there are a number of challenges and exciting prospects for the near future. On the theoretical side the main challenge will involve improving our control of strong interaction effects. We anticipate that in the coming years lattice QCD (in synergy with CHPT) will play an increasing role in determining the long-distance dynamics relevant for kaon decays. This will not only increase the accuracy of predictions for semileptonic modes, but will also put the SM predictions for nonleptonic decays on firmer ground, in both cases enhancing the constraining power of these decays with respect to models of New Physics. On the experimental side, the world-wide program aimed at rare decays will face the challenge of measuring branching ratios at the level of $10^{-11}$. This program will produce high-statistics results for other channels as a by-product, and has a great potential to discover effects of New Physics in the "golden modes" $K \rightarrow \pi \nu \bar{\nu}$. Even in the event of agreement with the SM predictions, these measurements will provide essential information on the flavor structure of SM extensions at the TeV scale. In both scenarios, kaons will play a central role at the low-energy frontier of our search for physics beyond the SM.

\section{Acknowledgments}

As documented in the bibliography, many physicists have contributed to the field of kaon decays. For their collaboration during the past 25 years, we thank especially J. Bijnens, F.J. Botella, A.G. Cohen, G. Colangelo, 
TABLE VII Comparison between theory (D'Ambrosio et al. , 1997) and experiment (Nakamura et al., 2010) for $K \rightarrow 3 \pi \gamma$ decays. The theoretical prediction for $\mathrm{BR}\left(K_{S} \rightarrow \pi^{+} \pi^{-} \pi^{0} \gamma\right)$ depends on NLO weak LECs and is a rough estimate only.

\begin{tabular}{|c|c|c|c|}
\hline & $E_{\gamma}^{\min }(\mathrm{MeV})$ & BR (theory) & BR (PDG'10) \\
\hline$K^{+} \rightarrow \pi^{0} \pi^{0} \pi^{+} \gamma$ & 10 & $(3.78 \pm 0.05) \times 10^{-6}$ & $\left(7.6_{-3.0}^{+6.0}\right) \times 10^{-6}$ \\
$K^{+} \rightarrow \pi^{+} \pi^{+} \pi^{-} \gamma$ & 5 & $(1.26 \pm 0.01) \times 10^{-4}$ & $(1.04 \pm 0.31) \times 10^{-4}$ \\
$K_{L} \rightarrow \pi^{+} \pi^{-} \pi^{0} \gamma$ & 10 & $(1.65 \pm 0.03) \times 10^{-4}$ & \\
$K_{S} \rightarrow \pi^{+} \pi^{-} \pi^{0} \gamma$ & 10 & $2 \times 10^{-10}$ & \\
\hline
\end{tabular}

G. D'Ambrosio, C.A. Dominguez, J.F. Donoghue, M. Eidemüller, J. Gasser, C. Gatti, M. Gianotti, E. Golowich, D. Gómez Dumm, M. González-Alonso, B. Guberina, G. Isidori, M. Jamin, J. Jenkins, R. Kaiser, J. Kambor, A. Kastner, M. Knecht, H. Leutwyler, K. Maltman, M. Moulson, G. Müller, S. Noguera, E. Pallante, M. Palutan, J.A. Peñarrocha, H. Pichl, J. Prades, E. de Rafael, I. Rosell, H. Rupertsberger, I. Scimemi, P. Talavera, R. Unterdorfer and D. Wyler. We also wish to thank B. Bloch-Devaux, A. Ceccucci, E. Cheu, M. Contalbrigo, E. Goudzovski, M. Raggi and C. Smith for correspondence. This work has been supported in part by the EU MRTN-CT-2006-035482 (FLAVIAnet). A. P. and J. P. are partially supported by MEC (Spain) under grant FPA2007-60323, by the Spanish Consolider-Ingenio 2010 Programme CPAN (CSD2007-00042) and by Generalitat Valenciana under grant PROMETEO/2008/069. J. P. is also partially supported by CSIC under grant PII-200750I026. The work of V.C. is supported by the Nuclear Physics Office of the U.S. Department of Energy under Contract No. DE-AC52-06NA25396 and by the LDRD program at Los Alamos National Laboratory.

\section{Appendix A: One-loop functions}

The following functions occur in the one-loop amplitudes for rare and radiative $K$ decays:

$$
\begin{aligned}
& F(z)=\left\{\begin{array}{ll}
1-\frac{4}{z} \arcsin ^{2}\left(\frac{\sqrt{z}}{2}\right) & z \leq 4 \\
1+\frac{1}{z}\left(\ln \frac{1-\sqrt{1-4 / z}}{1+\sqrt{1-4 / z}}+i \pi\right)^{2} & z>4
\end{array},\right. \\
& G(z)=\left\{\begin{array}{ll}
\sqrt{4 / z-1} \arcsin \left(\frac{\sqrt{z}}{2}\right) & z \leq 4 \\
\frac{1}{2} \sqrt{1-4 / z}\left(\ln \frac{1+\sqrt{1-4 / z}}{1-\sqrt{1-4 / z}}-i \pi\right) & z>4
\end{array}\right. \text {, } \\
& H(z)=\frac{1}{2(1-z)^{2}}\left\{z F\left(\frac{z}{r_{\pi}^{2}}\right)-F\left(\frac{1}{r_{\pi}^{2}}\right)\right. \\
& \left.-2 z\left[G\left(\frac{z}{r_{\pi}^{2}}\right)-G\left(\frac{1}{r_{\pi}^{2}}\right)\right]\right\}
\end{aligned}
$$

$$
\begin{aligned}
h_{i j}(z)=\frac{(4 \pi)^{2}}{z}[ & C_{20}\left(p^{2},(p+q)^{2}, M_{i}^{2}, M_{j}^{2}\right) \\
& \left.-C_{20}\left(p^{2}, p^{2}, M_{i}^{2}, M_{j}^{2}\right)\right],
\end{aligned}
$$

with $p^{2}=M_{\pi}^{2}$ (for $h_{\pi K}$ and $h_{K \eta}$ ) or $p^{2}=M_{K}^{2}\left(\right.$ for $\left.h_{\pi \pi}\right)$. $C_{20}$ is defined by the three-point integrals for $q^{2}=0$ :

$$
\begin{aligned}
& \int \frac{d^{4} \ell}{(2 \pi)^{4}} \frac{\ell^{\mu} \ell^{\nu}}{\left[\ell^{2}-M_{i}^{2}\right]\left[(\ell+q)^{2}-M_{i}^{2}\right]\left[(\ell-p)^{2}-M_{j}^{2}\right]} \\
& =i g^{\mu \nu} C_{20}\left(p^{2},(p+q)^{2}, M_{i}^{2}, M_{j}^{2}\right)+\ldots
\end{aligned}
$$

\section{References}

Abbas, G., B. Ananthanarayan, I. Caprini, and I. Sentitemsu Imsong, 2010, Phys. Rev. D82, 094018.

Abe, M., et al., 2006, Phys. Rev. D73, 072005.

Abouzaid, E., et al. (KTeV), 2006a, Phys. Rev. Lett. 96, 101801.

Abouzaid, E., et al. (KTeV), 2006b, Phys. Rev. D74, 032004. Abouzaid, E., et al. (KTeV), 2007a, Phys. Rev. D76, 052001. Abouzaid, E., et al. (KTeV), 2007b, Phys. Rev. Lett. 99, 051804 .

Abouzaid, E., et al. (KTeV), 2008a, Phys. Rev. D78, 032009. Abouzaid, E., et al. (KTeV), 2008b, Phys. Rev. D77, 112004. Abouzaid, E., et al. (KTeV), 2008c, Phys. Rev. D78, 032014. Abouzaid, E., et al. (KTeV), 2010, Phys. Rev. D81, 052001. Abouzaid, E., et al. (KTeV), 2011a, Phys. Rev. D83, 092001. Abouzaid, E., et al. (KTeV), 2011b, eprint 1105.4800.

Ademollo, M., and R. Gatto, 1964, Phys. Rev. Lett. 13, 264. Adeva, B., et al. (DIRAC), 2011, preprint CERN-PH-EP2011-028.

Adler, S. C., et al. (E787), 2000, Phys. Rev. Lett. 85, 2256.

Adler, S. C., et al. (E787), 2001, Phys. Rev. D63, 032004.

Ahn, J. K., et al. (E391a), 2008, Phys. Rev. Lett. 100, 201802. Ahn, J. K., et al. (E391a), 2010, Phys. Rev. D81, 072004.

Akimenko, S. A. (ISTRA+), 2007, Phys. Atom. Nucl. 70, 702.

Alavi-Harati, A., et al. (KTeV), 1999, Phys. Rev. Lett. 83, 22.

Alavi-Harati, A., et al. (KTeV), 2000a, Phys. Rev. D62, 112001.

Alavi-Harati, A., et al. (KTeV), 2000b, Phys. Rev. Lett. 84, 5279.

Alavi-Harati, A., et al. (KTeV), 2001a, Phys. Rev. D64, 012003.

Alavi-Harati, A., et al. (KTeV), 2001b, Phys. Rev. D64, 112004. 
Alavi-Harati, A., et al. (KTeV), 2001c, Phys. Rev. Lett. 87, 071801.

Alavi-Harati, A., et al. (KTeV), 2001d, Phys. Rev. Lett. 86, 5425.

Alavi-Harati, A., et al. (KTeV), 2001e, Phys. Rev. Lett. 86, 761.

Alavi-Harati, A., et al. (KTeV), 2002, Phys. Rev. Lett. 89, 211801.

Alavi-Harati, A., et al. (KTeV), 2003a, Phys. Rev. D67, 012005.

Alavi-Harati, A., et al. (KTeV), 2003b, Phys. Rev. Lett. 90, 141801.

Alavi-Harati, A., et al. (KTeV), 2004, Phys. Rev. Lett. 93, 021805.

Alexopoulos, T., et al. (KTeV), 2004, Phys. Rev. D70, 092007.

Alexopoulos, T., et al. (KTeV), 2005, Phys. Rev. D71, 012001.

Allton, C., et al. (RBC-UKQCD), 2008, Phys. Rev. D78, 114509.

Altarelli, G., and L. Maiani, 1974, Phys. Lett. B52, 351.

Ambrose, D., et al. (BNL E871), 1998, Phys. Rev. Lett. 81, 4309.

Ambrose, D., et al. (BNL E871), 2000, Phys. Rev. Lett. 84, 1389.

Ambrosino, F., et al. (KLOE), 2005, Phys. Lett. B619, 61.

Ambrosino, F., et al. (KLOE), 2007, JHEP 12, 105.

Ambrosino, F., et al. (KLOE), 2008, Eur. Phys. J. C55, 539.

Ambrosino, F., et al. (KLOE), 2009a, Eur. Phys. J. C64, 627.

Ambrosino, F., et al. (KLOE), 2009b, Phys. Lett. B672, 203.

Amelino-Camelia, G., F. Archilli, D. Babusci, D. Badoni, G. Bencivenni, et al., 2010, Eur. Phys. J. C68, 619.

Ametller, L., J. Bijnens, A. Bramon, and F. Cornet, 1993, Phys. Lett. B303, 140.

Amorós, G., and J. Bijnens, 1999, J. Phys. G25, 1607.

Amorós, G., J. Bijnens, and P. Talavera, 2000a, Nucl. Phys. B585, 293.

Amorós, G., J. Bijnens, and P. Talavera, 2000b, Phys. Lett. B480, 71.

Amorós, G., J. Bijnens, and P. Talavera, 2001, Nucl. Phys. B602, 87.

Ananthanarayan, B., G. Colangelo, J. Gasser, and H. Leutwyler, 2001, Phys. Rept. 353, 207.

Ananthanarayan, B., and B. Moussallam, 2004, JHEP 06, 047.

Antonelli, M., et al., 2010a, Eur. Phys. J. C69, 399.

Antonelli, M., et al., 2010b, Phys. Rept. 494, 197.

Antonelli, V., S. Bertolini, J. O. Eeg, M. Fabbrichesi, and E. I. Lashin, 1996a, Nucl. Phys. B469, 143.

Antonelli, V., S. Bertolini, M. Fabbrichesi, and E. I. Lashin, 1996b, Nucl. Phys. B469, 181.

Antonelli, V., S. Bertolini, M. Fabbrichesi, and E. I. Lashin, 1997, Nucl. Phys. B493, 281.

Aoki, S., et al. (PACS-CS), 2009, Phys. Rev. D79, 034503.

Aoki, Y., R. Arthur, T. Blum, P. Boyle, D. Brommel, et al., 2011, Phys. Rev. D84, 014503.

Artamonov, A. V., et al. (E949), 2008, Phys. Rev. Lett. 101, 191802.

Artuso, M., et al., 2008, Eur. Phys. J. C57, 309.

Aubin, C., J. Laiho, and R. S. Van de Water, 2008, PoS LATTICE2008, 105.

Aubin, C., J. Laiho, and R. S. Van de Water, 2010, Phys. Rev. D81, 014507.

Aubin, C., et al. (MILC), 2004, Phys. Rev. D70, 114501.
Bardeen, W. A., A. J. Buras, and J.-M. Gérard, 1987a, Phys. Lett. B192, 138.

Bardeen, W. A., A. J. Buras, and J.-M. Gérard, 1987b, Nucl. Phys. B293, 787.

Bardeen, W. A., A. J. Buras, and J.-M. Gérard, 1988, Phys. Lett. B211, 343.

Barker, A. R., H. Huang, P. A. Toale, and J. Engle, 2003, Phys. Rev. D67, 033008.

Barker, A. R., and S. H. Kettell, 2000, Ann. Rev. Nucl. Part. Sci. 50, 249

Barmin, V. V., et al., 1988, Sov. J. Nucl. Phys. 48, 1032.

Barmin, V. V., et al., 1991, Sov. J. Nucl. Phys. 53, 606.

Barr, G. D., et al. (NA31), 1993, Phys. Lett. B317, 233.

Batley, J. R., et al. (NA48), 2002, Phys. Lett. B544, 97.

Batley, J. R., et al. (NA48/1), 2003, Phys. Lett. B576, 43.

Batley, J. R., et al. (NA48), 2004a, Phys. Lett. B595, 75.

Batley, J. R., et al. (NA48/1), 2004b, Phys. Lett. B599, 197.

Batley, J. R., et al. (NA48/2), 2006, Phys. Lett. B633, 173.

Batley, J. R., et al. (NA48/2), 2007, Eur. Phys. J. C52, 875.

Batley, J. R., et al. (NA48/2), 2008a, Phys. Lett. B659, 493.

Batley, J. R., et al. (NA48/2), 2008b, Eur. Phys. J. C54, 411.

Batley, J. R., et al. (NA48/2), 2009a, Eur. Phys. J. C64, 589.

Batley, J. R., et al. (NA48/2), 2009b, Phys. Lett. B677, 246.

Batley, J. R., et al. (NA48/2), 2010a, Phys. Lett. B686, 101.

Batley, J. R., et al. (NA48/2), 2010b, Eur. Phys. J. C68, 75.

Batley, J. R., et al. (NA48/2), 2010c, Eur. Phys. J. C70, 635.

Batley, J. R., et al. (NA48/2), 2011a, Phys. Lett. B697, 107.

Batley, J. R., et al. (NA48/1), 2011b, Phys. Lett. B694, 301.

Battiston, R., D. Cocolicchio, G. L. Fogli, and N. Paver, 1992, Phys. Rept. 214, 293.

Bazavov, A., D. Toussaint, C. Bernard, J. Laiho, C. DeTar, et al., 2010, Rev. Mod. Phys. 82, 1349.

Bazavov, A., et al. (MILC), 2009, PoS CD09, 007.

Beane, S. R., P. F. Bedaque, K. Orginos, and M. J. Savage, 2007, Phys. Rev. D75, 094501.

Bećirević, D., et al., 2005, Nucl. Phys. B705, 339.

Behrends, R. E., and A. Sirlin, 1960, Phys. Rev. Lett. 4, 186.

Berends, F. A., A. Donnachie, and G. C. M. Oades, 1967, Phys. Lett. B26, 109.

Berends, F. A., A. Donnachie, and G. C. M. Oades, 1968, Phys. Rev. 171, 1457.

Bergström, L., E. Massó, and P. Singer, 1983, Phys. Lett. B131, 229.

Bergström, L., E. Massó, and P. Singer, 1990, Phys. Lett. B249, 141.

Bernard, C., et al., 2007, PoS LAT2007, 090.

Bernard, C. W., T. Draper, A. Soni, H. D. Politzer, and M. B. Wise, 1985, Phys. Rev. D32, 2343.

Bernard, V., D. R. Boito, and E. Passemar, 2011, eprint 1103.4855 .

Bernard, V., M. Oertel, E. Passemar, and J. Stern, 2006, Phys. Lett. B638, 480.

Bernard, V., M. Oertel, E. Passemar, and J. Stern, 2009, Phys. Rev. D80, 034034.

Bernard, V., and E. Passemar, 2008, Phys. Lett. B661, 95.

Bernard, V., and E. Passemar, 2010, JHEP 04, 001.

Bertolini, S., J. O. Eeg, and M. Fabbrichesi, 1995, Nucl. Phys. B449, 197.

Bertolini, S., J. O. Eeg, M. Fabbrichesi, and E. I. Lashin, 1998, Nucl. Phys. B514, 63.

Bertolini, S., M. Fabbrichesi, and J. O. Eeg, 2000, Rev. Mod. Phys. 72, 65.

Bhattacharya, T., et al., 2005, Nucl. Phys. Proc. Suppl. 140, 369. 
Bigi, I. I. Y., and A. I. Sanda, 2000, Camb. Monogr. Part. Phys. Nucl. Phys. Cosmol. 9, 1.

Bijnens, J., 1990, Nucl. Phys. B337, 635.

Bijnens, J., 2007, Prog. Part. Nucl. Phys. 58, 521.

Bijnens, J., and F. Borg, 2004, Nucl. Phys. B697, 319.

Bijnens, J., and F. Borg, 2005a, Eur. Phys. J. C39, 347.

Bijnens, J., and F. Borg, 2005b, Eur. Phys. J. C40, 383.

Bijnens, J., G. Colangelo, and G. Ecker, 1999, JHEP 02, 020.

Bijnens, J., G. Colangelo, and J. Gasser, 1994, Nucl. Phys. B427, 427.

Bijnens, J., P. Dhonte, and F. Borg, 2003, Nucl. Phys. B648, 317.

Bijnens, J., G. Ecker, and J. Gasser, 1993, Nucl. Phys. B396, 81.

Bijnens, J., G. Ecker, and A. Pich, 1992, Phys. Lett. B286, 341.

Bijnens, J., E. Gámiz, and J. Prades, 2001, JHEP 10, 009.

Bijnens, J., E. Gámiz, and J. Prades, 2004, Nucl. Phys. Proc. Suppl. 133, 245.

Bijnens, J., E. Gámiz, and J. Prades, 2006, JHEP 03, 048.

Bijnens, J., and K. Ghorbani, 2007a, JHEP 11, 030.

Bijnens, J., and K. Ghorbani, 2007b, eprint 0711.0148.

Bijnens, J., L. Girlanda, and P. Talavera, 2002, Eur. Phys. J. C23, 539.

Bijnens, J., and I. Jemos, 2011, eprint 1103.5945.

Bijnens, J., and J. Prades, 1995a, Phys. Lett. B342, 331.

Bijnens, J., and J. Prades, 1995b, Nucl. Phys. B444, 523.

Bijnens, J., and J. Prades, 1997, Nucl. Phys. B490, 239.

Bijnens, J., and J. Prades, 1999, JHEP 01, 023.

Bijnens, J., and J. Prades, 2000a, JHEP 06, 035.

Bijnens, J., and J. Prades, 2000b, JHEP 01, 002.

Bijnens, J., and P. Talavera, 2003, Nucl. Phys. B669, 341.

Bijnens, J., and M. B. Wise, 1984, Phys. Lett. B137, 245.

Birkfellner, W., 1996, Diploma Thesis, Univ. of Vienna.

Bissegger, M., A. Fuhrer, J. Gasser, B. Kubis, and A. Rusetsky, 2008, Phys. Lett. B659, 576.

Bissegger, M., A. Fuhrer, J. Gasser, B. Kubis, and A. Rusetsky, 2009, Nucl. Phys. B806, 178.

Blaser, S., 1995, Phys. Lett. B345, 287.

Bloch-Devaux, B. (NA48/2), 2011, in Proceedings of $46^{\text {th }}$ Rencontres de Moriond - QCD and High Energy Interactions, E. Augé et al., eds., The Gioi, Hanoi, 273.

Blossier, B., et al., 2009, JHEP 07, 043.

Blum, T., P. A. Boyle, N. H. Christ, N. Garron, E. Goode, et al., 2011, eprint 1106.2714.

Blum, T., et al. (RBC), 2003, Phys. Rev. D68, 114506.

Bolotov, V., et al. (KLOD), 2009, PoS KAON09, 052.

Bolotov, V. N., S. N. Gninenko, R. M. Dzhilkibaev, and V. V. Isakov, 1986, Sov. J. Nucl. Phys. 44, 68.

Bona, M., et al. (UTfit), 2006, JHEP 10, 081.

Bosch, S., et al., 2000, Nucl. Phys. B565, 3.

Boucaud, P., et al., 2005, Nucl. Phys. B721, 175.

Boyle, P., and N. Garron, 2010, PoS LATTICE2010, 307.

Boyle, P. A., et al., 2008, Phys. Rev. Lett. 100, 141601.

Boyle, P. A., et al., 2010, Eur. Phys. J. C69, 159.

Britton, D. I., S. Ahmad, D. A. Bryman, R. Burnham, E. Clifford, et al., 1994, Phys. Rev. D49, 28.

Britton, D. I., et al., 1992, Phys. Rev. Lett. 68, 3000.

Brod, J., and M. Gorbahn, 2008, Phys. Rev. D78, 034006.

Brod, J., and M. Gorbahn, 2010, Phys. Rev. D82, 094026.

Brod, J., M. Gorbahn, and E. Stamou, 2011, Phys. Rev. D83, 034030.

Brommel, D., et al. (QCDSF), 2007, PoS LAT2007, 364. Bruno, C., and J. Prades, 1993, Z. Phys. C57, 585.
Bryman, D., and R. Tschirhart, 2010, 'The Project X Kaon Physics Research Program', http://projectx.fnal.gov/.

Bryman, D. A., 1989, Int. J. Mod. Phys. A4, 79.

Buchalla, G., 2001, eprint hep-ph/0103166.

Buchalla, G., and A. J. Buras, 1994, Nucl. Phys. B412, 106.

Buchalla, G., and A. J. Buras, 1996, Phys. Rev. D54, 6782.

Buchalla, G., and A. J. Buras, 1998, Phys. Rev. D57, 216.

Buchalla, G., and A. J. Buras, 1999, Nucl. Phys. B548, 309.

Buchalla, G., A. J. Buras, and M. E. Lautenbacher, 1996, Rev. Mod. Phys. 68, 1125.

Buchalla, G., G. D'Ambrosio, and G. Isidori, 2003, Nucl. Phys. B672, 387.

Buchalla, G., and G. Isidori, 1998, Phys. Lett. B440, 170.

Buchholz, P., and B. Renk, 1997, Prog. Part. Nucl. Phys. 39, 253.

Büchler, M., 2006, Phys. Lett. B633, 497.

Budini, P., and L. Fonda, 1961, Phys. Rev. Lett. 6, 419.

Buras, A. J., 1996, eprint hep-ph/9609324.

Buras, A. J., 1998, in 'Probing the Standard Model of Particle Interactions', R. Gupta, A. Morel, E. de Rafael and F. David, eds., Elsevier Science B.V., Amsterdam, 281.

Buras, A. J., P. Gambino, M. Gorbahn, S. Jäger, and L. Silvestrini, 2001, Nucl. Phys. B592, 55.

Buras, A. J., and J.-M. Gérard, 1986, Nucl. Phys. B264, 371.

Buras, A. J., and J.-M. Gérard, 1987, Phys. Lett. B192, 156.

Buras, A. J., M. Gorbahn, U. Haisch, and U. Nierste, 2005, Phys. Rev. Lett. 95, 261805.

Buras, A. J., M. Gorbahn, U. Haisch, and U. Nierste, 2006, JHEP 11, 002.

Buras, A. J., and D. Guadagnoli, 2008, Phys. Rev. D78, 033005.

Buras, A. J., D. Guadagnoli, and G. Isidori, 2010, Phys. Lett. B688, 309.

Buras, A. J., and M. Jamin, 2004, JHEP 01, 048.

Buras, A. J., M. Jamin, and M. E. Lautenbacher, 1993a, Nucl. Phys. B408, 209.

Buras, A. J., M. Jamin, and M. E. Lautenbacher, 1996, Phys. Lett. B389, 749.

Buras, A. J., M. Jamin, M. E. Lautenbacher, and P. H. Weisz, 1993b, Nucl. Phys. B400, 37.

Buras, A. J., M. Jamin, and P. H. Weisz, 1990, Nucl. Phys. B347, 491.

Buras, A. J., M. E. Lautenbacher, M. Misiak, and M. Munz, 1994a, Nucl. Phys. B423, 349.

Buras, A. J., M. E. Lautenbacher, and G. Ostermaier, 1994b, Phys. Rev. D50, 3433.

Buras, A. J., F. Schwab, and S. Uhlig, 2008, Rev. Mod. Phys. 80, 965.

Burkhardt, H., et al. (NA31), 1988, Phys. Lett. B206, 169.

Cabibbo, N., 1963, Phys. Rev. Lett. 10, 531.

Cabibbo, N., 2004, Phys. Rev. Lett. 93, 121801.

Cabibbo, N., and G. Isidori, 2005, JHEP 03, 021.

Cabibbo, N., and A. Maksymowicz, 1965, Phys. Rev. 137, B438.

Cabibbo, N., and A. Maksymowicz, 1968, Phys. Rev. 168, 1926.

Callan, C. G., and S. B. Treiman, 1966, Phys. Rev. Lett. 16, 153.

Cappiello, L., and G. D'Ambrosio, 1988, Nuovo Cim. A99, 155.

Cappiello, L., and G. D'Ambrosio, 2007, Phys. Rev. D75, 094014.

Cappiello, L., G. D’Ambrosio, and M. Miragliuolo, 1993, Phys. Lett. B298, 423. 
Carroll, A. S., et al., 1980, Phys. Lett. B96, 407.

Charles, J., et al. (CKMfitter), 2005, Eur. Phys. J. C41, 1.

Charles, J., et al., 2010, http://ckmfitter.in2p3.fr/.

Cheng, H.-Y., 1983, Phys. Rev. D28, 150.

Cheng, H.-Y., 1990, Phys. Lett. B245, 122.

Chiang, C.-W., and F. J. Gilman, 2000, Phys. Rev. D62, 094026.

Chizhov, M. V., 2007, JHEP 0702, 045.

Christenson, J. H., J. Cronin, V. Fitch, and R. Turlay, 1964, Phys. Rev. Lett. 13, 138.

Cirigliano, V., J. F. Donoghue, E. Golowich, and K. Maltman, 2001, Phys. Lett. B522, 245.

Cirigliano, V., J. F. Donoghue, E. Golowich, and K. Maltman, 2003a, Phys. Lett. B555, 71.

Cirigliano, V., G. Ecker, H. Neufeld, and A. Pich, 2003b, JHEP 06, 012.

Cirigliano, V., G. Ecker, H. Neufeld, and A. Pich, 2004a, Eur. Phys. J. C33, 369.

Cirigliano, V., G. Ecker, and A. Pich, 2009, Phys. Lett. B679, 445.

Cirigliano, V., C. Gatti, M. Moulson, and M. Palutan (FlaviaNet Kaon Working Group), 2008a, eprint 0807.5128.

Cirigliano, V., M. Giannotti, and H. Neufeld, 2008b, JHEP 11, 006.

Cirigliano, V., and E. Golowich, 2000, Phys. Lett. B475, 351.

Cirigliano, V., and E. Golowich, 2002, Phys. Rev. D65, 054014.

Cirigliano, V., J. Jenkins, and M. González-Alonso, 2010, Nucl. Phys. B830, 95.

Cirigliano, V., M. Knecht, H. Neufeld, H. Rupertsberger, and P. Talavera, 2002, Eur. Phys. J. C23, 121.

Cirigliano, V., and H. Neufeld, 2011, Phys. Lett. B700, 7.

Cirigliano, V., H. Neufeld, and H. Pichl, 2004b, Eur. Phys. J. C35, 53.

Cirigliano, V., A. Pich, G. Ecker, and H. Neufeld, 2003c, Phys. Rev. Lett. 91, 162001.

Cirigliano, V., and I. Rosell, 2007a, JHEP 10, 005.

Cirigliano, V., and I. Rosell, 2007b, Phys. Rev. Lett. 99, 231801.

Cirigliano, V., et al., 2005, JHEP 04, 006.

Cirigliano, V., et al., 2006, Nucl. Phys. B753, 139.

Ciuchini, M., E. Franco, L. Giusti, V. Lubicz, and G. Martinelli, 1999, eprint hep-ph/9910237.

Ciuchini, M., E. Franco, G. Martinelli, and L. Reina, 1993, Phys. Lett. B301, 263.

Ciuchini, M., E. Franco, G. Martinelli, and L. Reina, 1994, Nucl. Phys. B415, 403.

Ciuchini, M., E. Franco, G. Martinelli, L. Reina, and L. Silvestrini, 1995, Z. Phys. C68, 239.

Ciuchini, M., et al., 1998, Nucl. Phys. B523, 501.

Cohen, A. G., G. Ecker, and A. Pich, 1993, Phys. Lett. B304, 347.

Colangelo, G., S. Dürr, A. Jüttner, L. Lellouch, H. Leutwyler, et al., 2011, Eur. Phys. J. C71, 1695.

Colangelo, G., J. Gasser, B. Kubis, and A. Rusetsky, 2006, Phys. Lett. B638, 187.

Colangelo, G., J. Gasser, and H. Leutwyler, 2000, Phys. Lett. B488, 261.

Colangelo, G., J. Gasser, and H. Leutwyler, 2001a, Nucl. Phys. B603, 125.

Colangelo, G., J. Gasser, and H. Leutwyler, 2001b, Phys. Rev. Lett. 86, 5008 .

Colangelo, G., J. Gasser, and A. Rusetsky, 2009, Eur. Phys. J. C59, 777 .
Colangelo, G., G. Isidori, and J. Portolés, 1999, Phys. Lett. B470, 134.

Collazuol, G. (NA62), 2009, PoS EPS-HEP2009, 260.

Cronin, J. A., 1967, Phys. Rev. 161, 1483.

Cuplov, V., and A. Nehme, 2003, eprint hep-ph/0311274.

Czapek, G., et al., 1993, Phys. Rev. Lett. 70, 17.

Dalitz, R. H., 1954, Phys. Rev. 94, 1046.

D'Ambrosio, G., G. Ecker, G. Isidori, and H. Neufeld, 1994, eprint hep-ph/9411439.

D'Ambrosio, G., G. Ecker, G. Isidori, and H. Neufeld, 1996, Phys. Lett. B380, 165.

D'Ambrosio, G., G. Ecker, G. Isidori, and H. Neufeld, 1997, Z. Phys. C76, 301.

D'Ambrosio, G., G. Ecker, G. Isidori, and J. Portolés, 1998a, JHEP 08, 004.

D'Ambrosio, G., and D. Espriu, 1986, Phys. Lett. B175, 237.

D'Ambrosio, G., and D.-N. Gao, 2000, JHEP 10, 043.

D'Ambrosio, G., and D.-N. Gao, 2002, JHEP 0207, 068.

D'Ambrosio, G., and G. Isidori, 1995, Z. Phys. C65, 649.

D'Ambrosio, G., and G. Isidori, 1998, Int. J. Mod. Phys. A13, 1.

D'Ambrosio, G., G. Isidori, and J. Portolés, 1998b, Phys. Lett. B423, 385.

D'Ambrosio, G., M. Miragliuolo, and F. Sannino, 1993, Z. Phys. C59, 451.

D'Ambrosio, G., M. Miragliuolo, and P. Santorelli, 1992, lNF92-066-P.

D'Ambrosio, G., and J. Portolés, 1996, Phys. Lett. B386, 403.

D'Ambrosio, G., and J. Portolés, 1997, Nucl. Phys. B492, 417.

D'Ambrosio, G., and J. Portolés, 1998a, Nucl. Phys. B533, 523.

D'Ambrosio, G., and J. Portolés, 1998b, Nucl. Phys. B533, 494.

Dashen, R. F., 1969, Phys. Rev. 183, 1245.

Dashen, R. F., and M. Weinstein, 1969, Phys. Rev. Lett. 22, 1337.

Dawson, C., T. Izubuchi, T. Kaneko, S. Sasaki, and A. Soni, 2006, Phys. Rev. D74, 114502.

Descotes-Genon, S., N. Fuchs, L. Girlanda, and J. Stern, 2002, Eur.Phys.J. C24, 469.

Descotes-Genon, S., and B. Moussallam, 2005, Eur. Phys. J. C42, 403.

Devlin, T. J., and J. O. Dickey, 1979, Rev. Mod. Phys. 51, 237.

Donoghue, J. F., and F. Gabbiani, 1995, Phys. Rev. D51, 2187.

Donoghue, J. F., and F. Gabbiani, 1997, Phys. Rev. D56, 1605.

Donoghue, J. F., and F. Gabbiani, 1998, Phys. Rev. D58, 037504 .

Donoghue, J. F., and E. Golowich, 2000, Phys. Lett. B478, 172 .

Donoghue, J. F., E. Golowich, and B. R. Holstein, 1984, Phys. Lett. B135, 481.

Donoghue, J. F., E. Golowich, and B. R. Holstein, 1992, Camb. Monogr. Part. Phys. Nucl. Phys. Cosmol. 2, 1.

Donoghue, J. F., B. R. Holstein, and Y. C. R. Lin, 1986, Nucl. Phys. B277, 651.

Donoghue, J. F., B. R. Holstein, and G. Valencia, 1987, Phys. Rev. D35, 2769.

Dubničková, A. Z., S. Dubnička, E. Goudzovski, V. N. Pervushin, and M. Sečansky, 2008, Phys. Part. Nucl. Lett. 5, 
76.

Duk, V. A., et al., 2011, Phys. Lett. B695, 59.

Dürr, S., Z. Fodor, C. Hoelbling, S. Katz, S. Krieg, et al., 2010, Phys. Rev. D81, 054507.

Dykstra, H., J. M. Flynn, and L. Randall, 1991, Phys. Lett. B270, 45.

Ebertshauser, T., H. W. Fearing, and S. Scherer, 2002, Phys. Rev. D65, 054033.

Ecker, G., 1995, Prog. Part. Nucl. Phys. 35, 1.

Ecker, G., 2007, Acta Phys. Polon. B38, 2753.

Ecker, G., J. Gasser, H. Leutwyler, A. Pich, and E. de Rafael, 1989a, Phys. Lett. B223, 425.

Ecker, G., J. Gasser, A. Pich, and E. de Rafael, 1989b, Nucl. Phys. B321, 311.

Ecker, G., G. Isidori, G. Müller, H. Neufeld, and A. Pich, 2000a, Nucl. Phys. B591, 419.

Ecker, G., J. Kambor, and D. Wyler, 1993, Nucl. Phys. B394, 101.

Ecker, G., G. Müller, H. Neufeld, and A. Pich, 2000b, Phys. Lett. B477, 88 .

Ecker, G., H. Neufeld, and A. Pich, 1992, Phys. Lett. B278, 337.

Ecker, G., H. Neufeld, and A. Pich, 1994, Nucl. Phys. B413, 321.

Ecker, G., and A. Pich, 1991, Nucl. Phys. B366, 189.

Ecker, G., A. Pich, and E. de Rafael, 1987a, Nucl. Phys. B291, 692.

Ecker, G., A. Pich, and E. de Rafael, 1987b, Phys. Lett. B189, 363.

Ecker, G., A. Pich, and E. de Rafael, 1988, Nucl. Phys. B303, 665.

Ecker, G., A. Pich, and E. de Rafael, 1990, Phys. Lett. B237, 481.

Ecker, G., and H. Pichl, 2001, Phys. Lett. B507, 193.

Efrosinin, V. P., I. B. Khriplovich, G. G. Kirilin, and Y. G. Kudenko, 2000, Phys. Lett. B493, 293.

Elwood, J. K., M. B. Wise, and M. J. Savage, 1995, Phys. Rev. D52, 5095.

Elwood, J. K., M. B. Wise, M. J. Savage, and J. W. Walden, 1996, Phys. Rev. D53, 4078.

Esposito-Farèse, G., 1991, Z. Phys. C50, 255.

Falk, A. F., A. Lewandowski, and A. A. Petrov, 2001, Phys. Lett. B505, 107.

Fanti, V., et al. (NA48), 1999, Phys. Lett. B465, 335.

Fearing, H. W., E. Fischbach, and J. Smith, 1970, Phys. Rev. D2, 542 .

Feldmann, T., and P. Kroll, 2002, Phys. Scripta T99, 13.

Finkemeier, M., 1996, Phys. Lett. B387, 391.

Flynn, J., and L. Randall, 1989a, Phys. Lett. B216, 221.

Flynn, J., and L. Randall, 1989b, Nucl. Phys. B326, 31.

Follana, E., C. T. H. Davies, G. P. Lepage, and J. Shigemitsu (HPQCD), 2008, Phys. Rev. Lett. 100, 062002.

Friot, S., D. Greynat, and E. de Rafael, 2004a, JHEP 10, 043.

Friot, S., D. Greynat, and E. de Rafael, 2004b, Phys. Lett. B595, 301.

Funck, R., and J. Kambor, 1993, Nucl. Phys. B396, 53.

Gabbiani, F., 1999, Phys. Rev. D59, 094022.

Gabbiani, F., and G. Valencia, 2002, Phys. Rev. D66, 074006.

Gaillard, M. K., and B. W. Lee, 1974a, Phys. Rev. Lett. 33, 108.

Gaillard, M. K., and B. W. Lee, 1974b, Phys. Rev. D10, 897.

Gaiser, B. D., T. Tsao, and M. B. Wise, 1981, Annals Phys. 132, 66.

Gámiz, E., J. Prades, and I. Scimemi, 2003, JHEP 10, 042.
Gámiz, E., J. Prades, and I. Scimemi, 2007, Eur. Phys. J. C50, 405.

Gao, D.-N., 2003, Phys. Rev. D67, 074028.

García-Martín, R., R. Kamiński, J. Peláez, J. Ruiz de Elvira, and F. Ynduráin, 2011, Phys. Rev. D83, 074004.

Gasser, J., 2004, Lect. Notes Phys. 629, U. G. Meißner and W. Plessas, eds., Springer, Heidelberg, 1.

Gasser, J., B. Kubis, N. Paver, and M. Verbeni, 2005, Eur. Phys. J. C40, 205.

Gasser, J., B. Kubis, and A. Rusetsky, 2011, Nucl. Phys. B850, 96.

Gasser, J., and H. Leutwyler, 1985a, Nucl. Phys. B250, 465.

Gasser, J., and H. Leutwyler, 1985b, Nucl. Phys. B250, 517.

Gasser, J., and G. Zarnauskas, 2010, Phys. Lett. B693, 122.

Gell-Mann, M., 1953, Phys. Rev. 92, 833.

Gell-Mann, M., 1961, 'The Eightfold Way: A Theory of Strong Interaction Symmetry', CALTECH Synchrotron Laboratory Report CTSL-20.

Geng, C. Q., I.-L. Ho, and T. H. Wu, 2004, Nucl. Phys. B684, 281.

Geng, C. Q., I. J. Hsu, and Y. C. Lin, 1994, Phys. Rev. D50, 5744.

Geng, C. Q., C. C. Lih, and C. C. Liu, 2000, Phys. Rev. D62, 034019.

Gérard, J.-M., C. Smith, and S. Trine, 2005, Nucl. Phys. B730, 1.

Gibbons, L. K., et al., 1993, Phys. Rev. Lett. 70, 1203.

Gilman, F. J., and M. B. Wise, 1979, Phys. Rev. D20, 2392.

Gilman, F. J., and M. B. Wise, 1980, Phys. Rev. D21, 3150.

Gilman, F. J., and M. B. Wise, 1983, Phys. Rev. D27, 1128.

Gjesdal, S., et al., 1973, Phys. Lett. B44, 217.

Glashow, S. L., 1961, Nucl. Phys. 22, 579.

Glashow, S. L., J. Iliopoulos, and L. Maiani, 1970, Phys. Rev. D2, 1285.

Göckeler, M., et al., 2006, PoS LAT2006, 179.

Goity, J. L., 1987, Z. Phys. C34, 341.

Gómez Dumm, D., and A. Pich, 1998, Phys. Rev. Lett. 80, 4633.

Goode, E. J., and M. Lightman, 2010, PoS LATTICE2010, 313.

Gorbahn, M., and U. Haisch, 2006, Phys. Rev. Lett. 97, 122002.

Greenlee, H. B., 1990, Phys. Rev. D42, 3724.

Grinstein, B., S.-J. Rey, and M. B. Wise, 1986, Phys. Rev. D33, 1495.

Guberina, B., A. Pich, and E. de Rafael, 1985, Phys. Lett. B163, 198.

Hambye, T., G. O. Köhler, E. A. Paschos, and P. H. Soldan, 2000, Nucl. Phys. B564, 391.

Hambye, T., G. O. Köhler, E. A. Paschos, P. H. Soldan, and W. A. Bardeen, 1998, Phys. Rev. D58, 014017.

Hambye, T., G. O. Köhler, and P. H. Soldan, 1999, Eur. Phys. J. C10, 271.

Hambye, T., S. Peris, and E. de Rafael, 2003, JHEP 05, 027. Hardy, J. C., and I. S. Towner, 2009, Phys. Rev. C79, 055502.

He, X. G., C. S. Huang, and X. Q. Li, 2003, Phys. Rev. D67, 096005.

Heiliger, P., B. McKellar, and L. M. Sehgal, 1994, Phys. Lett. B327, 145.

Heiliger, P., and L. M. Sehgal, 1993a, Phys. Rev. D47, 4920. Heiliger, P., and L. M. Sehgal, 1993b, Phys. Lett. B307, 182. Heiliger, P., and L. M. Sehgal, 1993c, Phys. Rev. D48, 4146. Herrera-Siklódy, P., J. I. Latorre, P. Pascual, and J. Tarón, 1998, Phys. Lett. B419, 326. 
Herrlich, S., and J. Kalinowski, 1992, Nucl. Phys. B381, 501. Herrlich, S., and U. Nierste, 1996, Nucl. Phys. B476, 27. Hill, R. J., 2006, Phys. Rev. D74, 096006.

Ho, S.-Y., and J. Tandean, 2010, Phys. Rev. D82, 114010.

Holstein, B. R., 1990, Phys. Rev. D41, 2829.

't Hooft, G., 1974a, Nucl. Phys. B72, 461.

't Hooft, G., 1974b, Nucl. Phys. B75, 461.

Inami, T., and C. S. Lim, 1981, Prog. Theor. Phys. 65, 297.

Isidori, G., F. Mescia, and C. Smith, 2005, Nucl. Phys. B718, 319.

Isidori, G., C. Smith, and R. Unterdorfer, 2004, Eur. Phys. J. C36, 57.

Isidori, G., and R. Unterdorfer, 2004, JHEP 01, 009.

Jamin, M., J. A. Oller, and A. Pich, 2002, Nucl. Phys. B622, 279.

Jamin, M., J. A. Oller, and A. Pich, 2004, JHEP 02, 047.

Jamin, M., J. A. Oller, and A. Pich, 2006, Phys. Rev. D74, 074009 .

Jamin, M., and A. Pich, 1994, Nucl. Phys. B425, 15.

Jiang, J.-H., D.-N. Gao, and M.-L. Yan, 2003, Mod. Phys. Lett. A18, 977.

Kaiser, R., and H. Leutwyler, 1998, eprint hep-ph/9806336.

Kambor, J., J. F. Donoghue, B. R. Holstein, J. H. Missimer, and D. Wyler, 1992, Phys. Rev. Lett. 68, 1818.

Kambor, J., and B. R. Holstein, 1994, Phys. Rev. D49, 2346.

Kambor, J., J. H. Missimer, and D. Wyler, 1990, Nucl. Phys. B346, 17.

Kambor, J., J. H. Missimer, and D. Wyler, 1991, Phys. Lett. B261, 496.

Kamiński, R., J. R. Peláez, and F. J. Ynduráin, 2008, Phys. Rev. D77, 054015.

Kampf, K., M. Knecht, J. Novotný, and M. Zdráhal, 2009, Nucl. Phys. Proc. Suppl. 186, 334.

Kastner, A., and H. Neufeld, 2008, Eur. Phys. J. C57, 541.

Kim, C. H., and C. T. Sachrajda, 2010, Phys. Rev. D81, 114506.

Kitching, P., et al. (E787), 1997, Phys. Rev. Lett. 79, 4079.

Knecht, M., H. Neufeld, H. Rupertsberger, and P. Talavera, 2000, Eur. Phys. J. C12, 469.

Knecht, M., S. Peris, M. Perrottet, and E. de Rafael, 1999a, Phys. Rev. Lett. 83, 5230.

Knecht, M., S. Peris, and E. de Rafael, 1999b, Phys. Lett. B457, 227.

Knecht, M., S. Peris, and E. de Rafael, 2001, Phys. Lett. B508, 117.

Kobayashi, M., and T. Maskawa, 1973, Prog. Theor. Phys. 49, 652.

Kohl, M. (J-PARC TREK), 2010, AIP Conf. Proc. 1265, 316.

Kubis, B., E. H. Müller, J. Gasser, and M. Schmid, 2007, Eur. Phys. J. C50, 557.

Kurshetsov, V. (OKA), 2009, PoS KAON09, 051.

Lai, A., et al. (NA48), 2001, Eur. Phys. J. C22, 231.

Lai, A., et al. (NA48), 2002, Phys. Lett. B536, 229.

Lai, A., et al. (NA48), 2003, Eur. Phys. J. C30, 33.

Lai, A., et al. (NA48), 2004, Phys. Lett. B578, 276.

Lai, A., et al. (NA48), 2005, Phys. Lett. B605, 247.

Lai, A., et al. (NA48), 2007, Phys. Lett. B647, 341.

Laiho, J., and R. S. Van de Water, 2010, PoS LATTICE2010, 312.

Lazzeroni, C., et al. (NA62), 2011, Phys. Lett. B698, 105.

Lee, T. D., and C.-N. Yang, 1956, Phys. Rev. 104, 254.

Lellouch, L., 2009, PoS LATTICE2008, 015.

Lellouch, L., and M. Lüscher, 2001, Commun. Math. Phys. 219, 31.
Leutwyler, H., 1996, Phys. Lett. B378, 313.

Leutwyler, H., 2009, PoS CD09, 005.

Leutwyler, H., and M. Roos, 1984, Z. Phys. C25, 91.

Li, L.-F., and L. Wolfenstein, 1980, Phys. Rev. D21, 178.

Lin, C. J. D., G. Martinelli, C. T. Sachrajda, and M. Testa, 2001, Nucl. Phys. B619, 467.

Lin, Y. C. R., and G. Valencia, 1988, Phys. Rev. D37, 143.

Littenberg, L. S., and G. Valencia, 1993, Ann. Rev. Nucl. Part. Sci. 43, 729.

Littenberg, L. S., and G. Valencia, 1996, Phys. Lett. B385, 379.

Liu, Q. (RBC, UKQCD), 2010, PoS LATTICE2010, 314.

Low, F. E., 1958, Phys. Rev. 110, 974.

Lu, M., and M. B. Wise, 1994, Phys. Lett. B324, 461.

Lubicz, V., F. Mescia, S. Simula, and C. Tarantino (ETM), 2009, Phys. Rev. D80, 111502.

Lusignoli, M., 1989, Nucl. Phys. B325, 33.

Ma, E., and J. Okada, 1978, Phys. Rev. D18, 4219.

Ma, E., and A. Pramudita, 1981, Phys. Rev. D24, 2476.

Makoff, G., et al., 1993, Phys. Rev. Lett. 70, 1591.

Marciano, W. J., 2004, Phys. Rev. Lett. 93, 231803.

Marciano, W. J., and Z. Parsa, 1996, Phys. Rev. D53, 1.

Marciano, W. J., and A. Sirlin, 1993, Phys. Rev. Lett. 71, 3629.

Martin, B. R., E. de Rafael, and J. Smith, 1970, Phys. Rev. D2, 179 .

Matthews, J. N., et al., 1995, Phys. Rev. Lett. 75, 2803.

Mawhinney, R. (RBC/UKQCD), 2009, PoS LAT2009, 081.

Mertens, P., and C. Smith, 2011, eprint 1103.5992.

Mescia, F., and C. Smith, 2007, Phys. Rev. D76, 034017.

Misiak, M., and J. Urban, 1999, Phys. Lett. B451, 161.

Morales, C. (NA48/2), 2008, in Proceedings of $43^{\text {rd }}$ Rencontres de Moriond - QCD and High Energy Interactions, The Gioi, Hanoi, eprint 0805.3312.

Morozumi, T., and H. Iwasaki, 1989, Prog. Theor. Phys. 82, 371.

Nakamura, K., et al. (PDG), 2010, J. Phys. G 37, 075021.

Nanjo, H. (K ${ }^{0}$ TO), 2009, PoS KAON09, 047.

Narison, S., 2001, Nucl. Phys. B593, 3.

Nehme, A., 2004a, Nucl. Phys. B682, 289.

Nehme, A., 2004b, Phys. Rev. D69, 094012.

Nehme, A., 2005, Eur. Phys. J. C40, 367.

Neufeld, H., and H. Rupertsberger, 1995, Z. Phys. C68, 91.

Noaki, J., et al. (TWQCD, JLQCD), 2009, PoS LAT2009, 096.

Noaki, J. I., et al. (CP-PACS), 2003, Phys. Rev. D68, 014501.

Ogata, R., et al. (E391a), 2011, eprint 1106.3404.

Okubo, S., 1962, Prog. Theor. Phys. 27, 949.

Okun, L. B., and I. B. Khriplovich, 1968, Sov. J. Nucl. Phys. 6, 598 .

Pais, A., 1952, Phys. Rev. 86, 663.

Pais, A., and S. B. Treiman, 1968a, Phys. Rev. 168, 1858.

Pais, A., and S. B. Treiman, 1968b, Phys. Rev. 176, 1974.

Pallante, E., and A. Pich, 2000, Phys. Rev. Lett. 84, 2568.

Pallante, E., and A. Pich, 2001, Nucl. Phys. B592, 294.

Pallante, E., A. Pich, and I. Scimemi, 2001, Nucl. Phys. B617, 441.

Paton, K., et al., 2006, J-PARC 50-GeV PS Experimental Proposal.

Pekurovsky, D., and G. Kilcup, 2001, Phys. Rev. D64, 074502.

Peris, S., and E. de Rafael, 2000, Phys. Lett. B490, 213.

Picciotto, C., 1992, Phys. Rev. D45, 1569.

Pich, A., 1995, Rept. Prog. Phys. 58, 563. 
Pich, A., 1998, eprint hep-ph/9806303.

Pich, A., 2002, Proc. Institute for Nuclear Theory (World Scientific) 12, 239, hep-ph/0205030.

Pich, A., 2004, eprint hep-ph/0410215.

Pich, A., B. Guberina, and E. de Rafael, 1986, Nucl. Phys. B277, 197.

Pich, A., and E. de Rafael, 1985, Phys. Lett. B158, 477.

Pich, A., and E. de Rafael, 1987, Phys. Lett. B189, 369.

Pich, A., and E. de Rafael, 1991, Nucl. Phys. B358, 311.

Pich, A., and E. de Rafael, 1996, Phys. Lett. B374, 186.

Pichl, H., 2001, Eur. Phys. J. C20, 371.

Pislak, S., et al. (BNL-E865), 2001, Phys. Rev. Lett. 87, 221801.

Pislak, S., et al. (BNL-E865), 2003, Phys. Rev. D67, 072004.

Poblaguev, A. A., et al., 2002, Phys. Rev. Lett. 89, 061803.

Post, P., and K. Schilcher, 2002, Eur. Phys. J. C25, 427.

Počanić, D., et al. (PEN), 2009, AIP Conf. Proc. 1182, 698.

Prades, J., 2008, PoS KAON, 022.

Prades, J., C. A. Dominguez, J. A. Peñarrocha, A. Pich, and E. de Rafael, 1991, Z. Phys. C51, 287.

Prades, J., E. Gámiz, and I. Scimemi, 2007, Nucl. Phys. Proc. Suppl. 164, 79.

Pramudita, A., 1988, Phys. Rev. D38, 3522.

de Rafael, E., 1995, 'CP Violation and the Limits of the Standard Model', J. F. Donoghue, ed., World Scientific, Singapore, eprint hep-ph/9502254.

Ramberg, E. J., et al. (E731), 1993, Phys. Rev. Lett. 70, 2529.

Richardson, S., and C. Picciotto, 1995, Phys. Rev. D52, 6342.

Riggenbach, C., J. Gasser, J. F. Donoghue, and B. R. Holstein, 1991, Phys. Rev. D43, 127.

Ritchie, J. L., and S. G. Wojcicki, 1993, Rev. Mod. Phys. 65, 1149.

Rochester, G. D., and C. Butler, 1947, Nature 160, 855.

Rosselet, L., et al., 1977, Phys. Rev. D15, 574.

Roy, S. M., 1971, Phys. Lett. B36, 353.

Salam, A., 1968, in Proceedings of the $8^{\text {th }}$ Nobel Symposium,

N. Svartholm, ed., Almqvist and Wiksell, Stockholm, 367.

Scherer, S., 2003, Adv. Nucl. Phys. 27, 277.

Sehgal, L. M., 1969, Phys. Rev. 181, 2151.

Sehgal, L. M., 1973, Phys. Rev. D7, 3303.

Sehgal, L. M., 1988, Phys. Rev. D38, 808.
Sehgal, L. M., 1990, Phys. Rev. D41, 161.

Sehgal, L. M., and J. van Leusen, 1999, Phys. Rev. Lett. 83, 4933.

Sehgal, L. M., and J. van Leusen, 2000, Phys. Lett. B489, 300.

Sehgal, L. M., and M. Wanninger, 1992, Phys. Rev. D46, 1035.

Sharpe, S. R., 1987, Phys. Lett. B194, 551.

Sher, A., M. Aoki, M. Blecher, D. A. Bryman, J. Comfort, et al., 2009, AIP Conf.Proc. 1182, 702.

Shifman, M. A., A. I. Vainshtein, and V. I. Zakharov, 1977, Nucl. Phys. B120, 316.

Shimizu, S., et al. (KEK-E470), 2004, Phys. Rev. D70, 037101.

Sirlin, A., 1978, Rev. Mod. Phys. 50, 573.

Sirlin, A., 1982, Nucl. Phys. B196, 83.

Spadaro, T. (NA62), 2011, eprint 1101.5631.

Tandean, J., and G. Valencia, 2000, Phys. Rev. D62, 116007.

Tchikilev, O., et al., 2010, eprint 1001.0374.

Tschirhart, R., 2011, Nucl. Phys. Proc. Suppl. 210-211, 220.

Tsutsui, N., et al. (JLQCD), 2006, PoS LAT2005, 357.

Tung, Y., et al. (E391a), 2011, Phys. Rev. D83, 031101.

Unterdorfer, R., and G. Ecker, 2005, JHEP 0510, 017.

Urech, R., 1995, Nucl. Phys. B433, 234.

Vainshtein, A. I., V. I. Zakharov, and M. A. Shifman, 1975, JETP Lett. 22, 55.

Watanabe, H. (J-PARC E14 KOTO), 2010, PoS ICHEP2010, 274.

Webber, D. M., et al. (MuLan), 2011, Phys. Rev. Lett. 106, 041803.

Weinberg, S., 1967, Phys. Rev. Lett. 19, 1264.

Weinberg, S., 1979, Physica A96, 327.

Wess, J., and B. Zumino, 1971, Phys. Lett. B37, 95.

Winstein, B., and L. Wolfenstein, 1993, Rev. Mod. Phys. 65, 1113.

Witten, E., 1979, Nucl. Phys. B160, 57.

Witten, E., 1983, Nucl. Phys. B223, 422.

Wolfenstein, L., 1983, Phys. Rev. Lett. 51, 1945.

Yushchenko, O. P., et al., 2004, Phys. Lett. B581, 31.

Zhitnitsky, A. R., 1980, Sov. J. Nucl. Phys. 31, 529. 